\title{
Epicardial amiodarone therapy for atrial fibrillation : delivery methods, drug distribution and antiarrhythmic effects
}

Citation for published version (APA):

Bolderman, R. W. (2012). Epicardial amiodarone therapy for atrial fibrillation : delivery methods, drug distribution and antiarrhythmic effects. [Doctoral Thesis, Maastricht University]. Datawyse / Universitaire Pers Maastricht. https://doi.org/10.26481/dis.20120420rb

Document status and date:

Published: 01/01/2012

DOI:

10.26481/dis.20120420rb

Document Version:

Publisher's PDF, also known as Version of record

\section{Please check the document version of this publication:}

- A submitted manuscript is the version of the article upon submission and before peer-review. There can be important differences between the submitted version and the official published version of record.

People interested in the research are advised to contact the author for the final version of the publication, or visit the DOI to the publisher's website.

- The final author version and the galley proof are versions of the publication after peer review.

- The final published version features the final layout of the paper including the volume, issue and page numbers.

Link to publication

\footnotetext{
General rights rights.

- You may freely distribute the URL identifying the publication in the public portal. please follow below link for the End User Agreement:

www.umlib.nl/taverne-license

Take down policy

If you believe that this document breaches copyright please contact us at:

repository@maastrichtuniversity.nl

providing details and we will investigate your claim.
}

Copyright and moral rights for the publications made accessible in the public portal are retained by the authors and/or other copyright owners and it is a condition of accessing publications that users recognise and abide by the legal requirements associated with these

- Users may download and print one copy of any publication from the public portal for the purpose of private study or research.

- You may not further distribute the material or use it for any profit-making activity or commercial gain

If the publication is distributed under the terms of Article $25 \mathrm{fa}$ of the Dutch Copyright Act, indicated by the "Taverne" license above, 


\section{EPICARDIAL AMIODARONE THERAPY FOR ATRIAL FIBRILLATION}

DELIVERY METHODS, DRUG DISTRIBUTION AND ANTIARRHYTHMIC EFFECTS 
(c) R.W. Bolderman, Maastricht 2012

ISBN 9789461590992

Cover illustration based on "The Creation of Adam" by Michelangelo Printed by Datawyse I Universitaire Pers Maastricht 


\title{
EPICARDIAL AMIODARONE THERAPY FOR ATRIAL FIBRILLATION
}

\author{
DELIVERY METHODS, DRUG DISTRIBUTION \\ AND ANTIARRHYTHMIC EFFECTS
}

\section{PROEFSCHRIFT}
Ter verkrijging van de graad van doctor aan de Universiteit Maastricht, op gezag van de Rector Magnificus, Prof. mr. G.P.M.F. Mols, volgens het besluit van het College van Decanen,
in het openbaar te verdedigen
op vrijdag 20 april 2012 om 14:00 uur

door

\section{Robert Willem Bolderman}

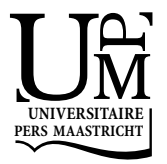




\section{Promotor}

Prof. dr. J.G. Maessen

\section{Copromotor}

Dr. J.J.R. Hermans

\section{Beoordelingscommissie}

Prof. dr. F.W. Prinzen (voorzitter)

Prof. dr. H.J.G.M. Crijns

Prof. dr. J.G.R. de Mey

Prof. dr. A. zur Hausen

Financial support by Stichting Hartsvrienden RESCAR and the Netherlands Heart Foundation for the publication of this thesis is gratefully acknowledged.

Additional financial support was kindly provided by DSM Biomedical, Merck Sharp \& Dohme B.V. and Servier Nederland Farma B.V. 


\section{PREFACE}

Atrial fibrillation (AF) is the most common cardiac arrhythmia requiring medical attention, affecting $4 \%$ of the population over 60 years, increasing with age to $8 \%$ in those older than 80 years. It is important clinically because patients are at increased risk for stroke, heart failure and mortality (almost two-fold compared with patients in normal sinus rhythm). In addition, AF is associated with a significant morbidity, impairing both functional status and quality of life. The economic burden of AF is a growing public health problem, approaching $€ 3000$ annually per patient. Total cost burden is likely to increase over the next years due to the aging of the population and the rising prevalence of patients with cardiovascular disease. AF is also one of the most frequent complications of cardiac surgery, resulting in increased patient morbidity, prolonged hospitalization and increased hospital costs.

There are three main objectives of AF treatment: rhythm control, ventricular rate control, and prevention of thromboembolism. Although nonpharmacological therapies, such as surgical ablation, catheter ablation, pacemakers and internal defibrillators, have been developed, pharmacotherapy is still the mainstay of AF treatment. However, currently available drugs for rhythm control are only moderately effective and all carry risks of serious side effects, most notably ventricular arrhythmias. Of these drugs, amiodarone is the most frequently used agent worldwide for maintaining sinus rhythm in patients with AF. It displays superior efficacy combined with a low potential for causing torsades de pointes. Unfortunately, amiodarone therapy is also associated with numerous systemic side effects, including thyroid, pulmonary, hepatic and dermatologic toxicity.

The search for a novel antiarrhythmic agent sharing amiodarone's efficacy, but devoid of organ toxicity is regarded as a search for the Holy Grail. Therefore, several amiodarone analogues have been synthesized, of which dronedarone is most advanced in clinical development. Recent trials suggest that dronedarone has fewer side effects, but its efficacy at maintaining sinus rhythm is probably less than that of amiodarone. This thesis explores an alternative approach to maintain amiodarone's unique antiarrhythmic properties, while minimizing its systemic adverse effects, through the use of intrapericardial drug delivery and epicardial drug-releasing biomaterials. 



\section{CONTENTS}

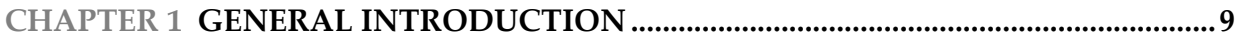

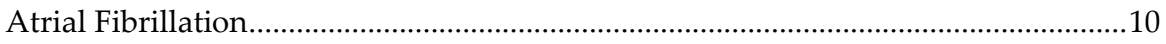

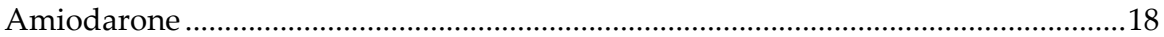

Intrapericardial and Epicardial Pharmacotherapy .................................................22

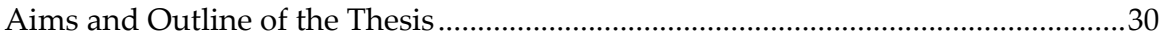

CHAPTER 2 DETERMINATION OF AMIODARONE AND

DRONEDARONE, AND THEIR PRINCIPAL METABOLITES IN

PLASMA AND MYOCARDIUM BY HIGH-PERFORMANCE LIQUID

CHROMATOGRAPHY AND UV-DETECTION

CHAPTER 3 INTRAPERICARDIAL DELIVERY OF AMIODARONE AND

SOTALOL: ATRIAL TRANSMURAL DRUG DISTRIBUTION AND

ELECTROPHYSIOLOGICAL EFFECTS

CHAPTER 4 EPICARDIAL APPLICATION OF AN AMIODARONE-

RELEASING HYDROGEL TO SUPPRESS ATRIAL

TACHYARRHYTHMIAS

CHAPTER 5 ATRIUM-TARGETED DRUG DELIVERY THROUGH AN AMIODARONE-ELUTING BILAYERED PATCH

CHAPTER 6 EFFECTS OF EPICARDIAL AMIODARONE DELIVERY ON TRANSMURAL ATRIAL ELECTROPHYSIOLOGY AND SUSTAINED ATRIAL FIBRILLATION

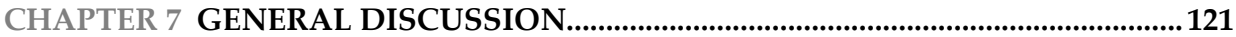

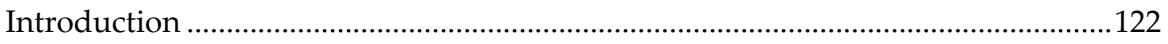

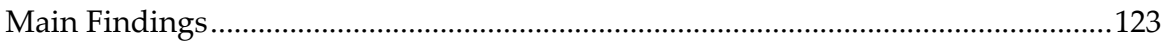

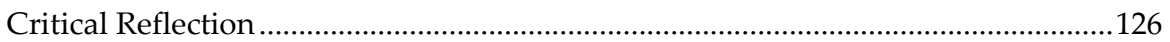

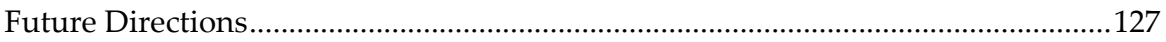

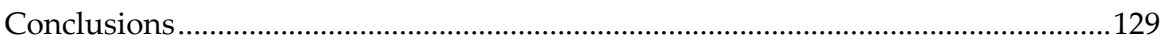

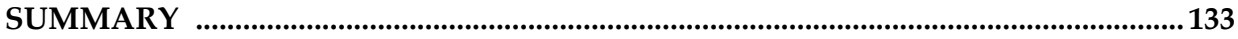

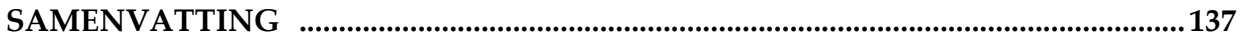

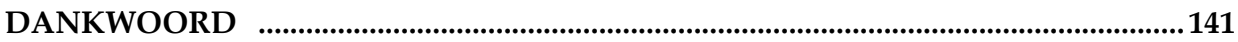

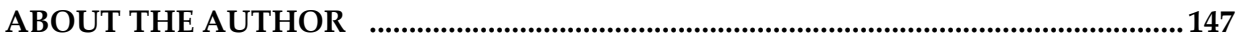

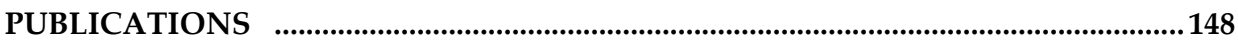



CHAPTER 1

General Introduction 


\section{Atrial Fibrillation}

In 1906, Cushny and Edmunds were the first to report that "auricular fibrillation", previously observed in dogs during open-chest experiments, could occur in patients. ${ }^{1}$ In the same year, Einthoven published a review article on the clinical value of the electrocardiograph, including the first clinical electrocardiographic tracing displaying "pulsus inaequalis et irregularis". ${ }^{2}$ In 1902, Mackenzie already observed jugular pulse tracings with frequent ventricular components, but missing atrial components. ${ }^{3}$ A few years later, Cushny acknowledged that Mackenzie's article had provided him the insight that led to the 1906 report, while Mackenzie admitted that Cushny suggested to him that atrial fibrillation might be of clinical importance. ${ }^{4}$ The latter was confirmed in 1909, when Rothberger and Winterberg, as well as Lewis independently concluded that atrial fibrillation was a common arrhythmia in patients with cardiac disease. 5,6

\subsection{Clinical Aspects}

\subsubsection{Definition and Classification}

Atrial fibrillation (AF) is a supraventricular tachyarrhythmia characterized by rapid and irregular activation of the atrium. ${ }^{7}$ Instead of contracting, the atria only quiver, which leads to deterioration of mechanical function. The filtering function of the atrioventricular (AV) node determines the ventricular response rate, which typically approaches 150 beats per minute in the absence of drug therapy. ${ }^{8}$

International guidelines for the management of patients with AF recommend the following classification.9-11 The clinician should distinguish a first-detected episode of AF from recurrent AF (after 2 or more confirmed episodes). AF can be further designated as paroxysmal (episodes shorter than 7 days), persistent (sustained episodes beyond 7 days) or permanent (long-standing AF, impossible to cardiovert or cardioversion not attempted). Secondary AF occurs in the setting of an underlying disease, while lone $\mathrm{AF}$ is defined as $\mathrm{AF}$ in individuals younger than 60 years without underlying cardiopulmonary disorders (including hypertension). ${ }^{12}$

\subsubsection{Epidemiology and Prognosis}

Atrial fibrillation is the most common cardiac arrhythmia requiring medical attention. Its estimated prevalence is $0.4 \%$ to $1 \%$ in the general population, $4 \%$ in the population over 60 years of age and $9 \%$ in the octogenarian population. ${ }^{13-15}$ The incidence of $\mathrm{AF}$, in line with its prevalence, increases with advancing age from 0.5 per 1000 person-years before age 50 to 10 per 1000 person-years after age $70 .{ }^{16}$ During the past 2 decades, there has been a $60 \%$ increase in hospital admissions for AF 
due to multiple factors including aging of the population, rising prevalence of cardiovascular disease and improved diagnostic methods. ${ }^{17}$

AF is associated with increased stroke, heart failure and mortality in otherwise healthy older individuals and those with coexisting cardiovascular disease, especially among women. ${ }^{13,18-22}$ In the Framingham Heart Study the risk of death in both men and women with AF was almost doubled, while odds ratios after adjustment for AF-associated preexisting cardiovascular disease were 1.5 and 1.9 for men and woman, respectively. ${ }^{13} \mathrm{AF}$ is an important independent risk factor for stroke, with an attributable risk increasing with age from $1.5 \%$ for those aged 50-59 years to $23.5 \%$ for those aged $80-89$ years. ${ }^{18}$

\subsubsection{Causes and Associations}

In $55 \%$ to $70 \%$ of cases of paroxysmal $\mathrm{AF}$ and in $80 \%$ to $85 \%$ of cases with persistent AF underlying disease, most often cardiopulmonary disorders, can be identified. ${ }^{23}$ These include pericarditis, ${ }^{24}$ myocarditis, ${ }^{25}$ other primary arrhythmias, ${ }^{26}$ valvular heart disease, ${ }^{27}$ heart failure ${ }^{28}$ coronary artery disease, ${ }^{29,30}$ hypertension, ${ }^{16}$ hypertrophic cardiomyopathy, ${ }^{31}$ congenital heart disease, ${ }^{32}$ pulmonary embolism, ${ }^{33}$ and obstructive pulmonary disease. ${ }^{34} \mathrm{AF}$ is also associated with hyperthyroidism, ${ }^{35}$ sleep apnea syndrome, ${ }^{36}$ and obesity. ${ }^{37}$ AF can be provoked by alcohol intake (binge drinking $)^{38}$ and electrocution, ${ }^{39}$ and is a common complication of cardiac and thoracic surgery. ${ }^{40}$ In addition, inflammatory, ${ }^{41}$ autonomic, ${ }^{42}$ and genetic factors ${ }^{43}$ may contribute to the initiation and perpetuation of AF.

\subsubsection{Manifestation and Evaluation}

Most patients with first-detected AF complain of palpitations, chest pain, dyspnea, fatigue or lightheadedness. However, embolic complications, exacerbation of heart failure or syncope may also represent the first signs of AF. Individuals can experience both symptomatic and asymptomatic AF episodes. ${ }^{44}$ This should be taken into account when determining frequency and duration of AF episodes from the patient's history. Next to characterizing the clinical pattern of $\mathrm{AF}$, the purpose of the history and physical examination should be to define any causes or influencing factors. The diagnosis of AF requires confirmation by ECG recording. Other necessary investigations are a chest radiograph, a transthoracic echocardiogram and a blood test (for determining blood count, serum electrolytes, and thyroid, renal and hepatic function). ${ }^{9-11}$

\subsubsection{Current Treatment Strategies and Nonpharmacological Therapies}

There are three main objectives of AF treatment: restoration and maintenance of sinus rhythm, prevention of rapid ventricular rates, and prevention of thromboembolism. Antiarrhythmic pharmacotherapy of AF is discussed in detail in section 1.3. 
Nonpharmacological therapies for AF include synchronized external direct-current cardioversion, atrial and ventricular pacing, internal atrial defibrillators, surgical cut-and-sew ablation, surgical epicardial ablation and catheter-based ablation. Electrical cardioversion, although requiring sedation or anesthesia, may be preferred over pharmacological cardioversion because of greater effectiveness and a low risk of proarrhythmia. ${ }^{45}$ Ventricular and atrial pacing may be useful in the settings of marked variability in ventricular rates and symptomatic bradycardia, respectively.46,47 Internal atrial defibrillators produce a high rate of conversion to sinus rhythm, but the discharges cause discomfort in many patients. ${ }^{48}$ Surgical cut-andsew ablation (maze III procedure) has success rates of $70 \%$ to $95 \% .{ }^{49,50}$ However, the complexicity and the relatively high risk associated with this procedure have limited its widespread use. Surgical epicardial ablation does not require cardiopulmonary bypass and may provide a safer alternative, although its role amidst other ablation techniques is yet to be determined. Most catheter-based ablation techniques focus on isolating ectopic foci in the pulmonary veins from the left atrium, thereby preventing further atrial remodeling. ${ }^{51}$ Current available data suggest that these techniques offer benefit to selected patients with AF. However, reported rates of success and complications vary considerably. Long-term results of double-blind randomized trials are needed to properly compare safety and effectiveness with pharmacological therapies for AF.

\subsection{Electrophysiological Mechanisms}

This section discusses the electrophysiological basis of initiation and perpetuation of AF. Sources responsible for initiation of AF are known as "triggers". When a rapid source dominates during perpetuation of AF, it is termed "driver". Other mechanisms that facilitate the perpetuation of AF constitute the atrial "substrate". Whereas classical theories focused on the role of triggers and drivers, the discovery of the phenomenon of "AF begets AF" spurred interest into the role of atrial remodeling in generating a substrate for AF.52 However, research on triggers and drivers has recently made a revival after the demonstration of the importance of pulmonary vein foci in initiating AF. ${ }^{51}$

\subsubsection{Classical Theories}

In the early twentieth century 3 principal theories were developed: AF is caused by rapidly-firing atrial ectopic foci, by a single reentry circuit, or by multiple reentrant circuits. ${ }^{53}$ The hypothesis that AF may originate from ectopic foci was already proposed in 1907, but experimental evidence was provided only 40 years later by the application of aconitine (a sodium channel opener) to canine atria. ${ }^{54,55}$ First support for a reentrant mechanism came in 1906 from experiments with muscular rings from 
jelly fish, showing circus movement of an activation wavefront. ${ }^{56}$ However, the ectopic focus and single reentry circuit theories were largely abandoned in the midtwentieth century in favour of the "multiple wavelet hypothesis". .57 The existence of multiple propagating wave fronts was confirmed in the first mapping studies of AF. ${ }^{58}$ An important contribution to the understanding of reentry and AF is the notion of "wavelength", which is, according to the "leading circle hypothesis", the minimum pathlength that can sustain reentry. ${ }^{59}$

\subsubsection{Atrial Remodeling}

The concept of "AF begets AF" was introduced in 1995 based on experiments in goats. ${ }^{52} \mathrm{AF}$ was maintained by an automatic fibrillation pacemaker, activating at the occurence of sinus rhythm. This resulted in a marked shortening of atrial effective refractory period ("electrical remodeling") and an increase in inducibility and stability of AF. The latter was explained by a shortening of the wavelength. Tachycardiainduced shortening of atrial refractoriness was also observed in a dog model of prolonged rapid atrial pacing. ${ }^{60}$ An important ionic mechanism underlying electrical remodeling is a decrease in L-type calcium current in response to cellular calcium loading. ${ }^{61,62}$ In addition to electrical remodeling, clinical studies have demonstrated AF-induced atrial contractile dysfunction ("contractile remodeling").63,64 In goats electrical and contractile remodeling were closely linked during the first 5 days of artificially maintained $\mathrm{AF}$, suggesting that a decrease in L-type calcium current might also play an important role in atrial contractile dysfunction. ${ }^{65}$

Besides electrical and contractile remodeling, AF also causes ultrastructural changes, termed "structural remodeling". First evidence came from studies in a canine model of rapid atrial pacing, showing disarray of atrial myofilaments, increased mitochondrial size and number, and disruption of the sarcoplasmatic reticulum. ${ }^{60}$ Other prominent atrial structural alterations include myolysis, increase in myocyte size and perinuclear accumulation of glycogen, which develop progressively and recover slowly and imcompletely ${ }^{66,67}$ Increased atrial interstitial fibrosis is one of the most frequent histopathological changes in AF, but this is probably related to older age and underlying heart disease. ${ }^{68,69}$ Fibrosis increases non-uniform anisotropy in conduction and, therefore, promotes the substrate for local reentry. ${ }^{70}$ Conduction heterogeneity may be further enhanced by alterations in distribution of gap junction proteins (connexins)..$^{71,72}$ Recent mapping studies have shown that the structural remodeling process during AF may lead to progressive electrical dissociation between individual atrial muscle bundles in the epicardial plane and even between the epicardial and endocardial layers. ${ }^{73-75}$ 


\subsubsection{Pulmonary Vein Foci}

The importance of pulmonary vein foci in initiating AF was recognized in 1998, when it was demonstrated that ablation of these foci could prevent AF recurrence. ${ }^{51}$ Ectopic foci have also been located in other cardiac venes and in both atrial walls, although the myocardial sleeves in the pulmonary veins are the primary source. ${ }^{51,76-78}$ The underlying mechanisms of pulmonary vein activity remain poorly understood. Two hypotheses have been proposed; disarrayed fibers of the myocardial sleeves promote micro-reentry or they harbour cells featuring spontaneous firing. ${ }^{79,80}$ The former theory is supported by the recent observation that adenosine (via enhancement of inward rectifier conductance) increases dominant frequencies of AF drivers near the pulmonary vein-left atrial junction. ${ }^{81}$ Evidence suggesting spontaneous activity is the fact that pulmonary vein firing is often preceeded by a tachycardia-pause sequence, which may be explained by a "calcium-transient triggering" mechanism. ${ }^{82}$

\subsection{Antiarrhythmic Pharmacotherapy}

In the absence of antiarrhythmic pharmacotherapy the ventricular response rate in AF typically approaches 150 beats per minute. The management of this fast irregular rhythm involves either a rate control or rhythm control strategy. The rhythm control strategy aims to restore and maintain normal sinus rhythm. The rate control strategy attempts to slow the ventricular rate while leaving the patient in AF. The choice between the two strategies has been the subject of extensive debate.

\subsubsection{Rate Control}

Until 2 decades ago rhythm control was the mainstay of AF management. However, several studies that compared rate and rhythm control found no difference between the strategies in terms of mortality, major cardiovascular events, or stroke. ${ }^{83-87}$ This might be related to the moderate effectiveness and proarrhythmic characteristics of currently available drugs for rhythm control. Therefore, rate control is an acceptable first line treatment in many AF patients, especially in asymptomatic older patients with persistent AF who have hypertension or heart disease. Although the 2006 AHA/ESC/ACC guidelines recommend a resting heart rate of $60-80$ beats per minute, a recent study suggested that a more lenient rate control (resting heart rate $<110$ beats per minute) is equally effective and is easier to achieve. ${ }^{88}$

The most common drugs used for controlling heart rate are beta blockers, nondihydropyridine calcium channel blockers and digoxin. Digoxin is less effective at controlling heart rate during exercise and other settings of elevated sympathetic tone. ${ }^{89,90}$ When the ventricular rate cannot be adequately controlled using these drugs (alone or in combination) amiodarone may be considered to control the heart 
rate. ${ }^{91}$ In patients with decompensated heart failure, acute use of nondihydropyridine calcium channel blockers or large doses of beta blockers shoud be avoided as both are negative inotropic drugs..$^{90,92}$

\subsubsection{Rhythm Control}

Rhythm control may be a reasonable initial approach for younger patients, especially those with symptomatic paroxysmal lone AF. Traditional antiarrhythmic drugs used for rhythm control are sodium and potassium channel blockers (class I and III antiarrhythmic agents respectively, according to the Vaughan Williams classification). ${ }^{93}$ Class I agents slow conduction and suppress ectopic activity associated with early and delayed afterdepolarisation. These actions probably destabilise spiral-wave AF-drivers and suppress AF-triggers. ${ }^{94}$ The conduction-slowing effect is more pronounced in ischaemic ventricular tissue, increasing the risk of ventricular proarrhythmia in patients with coronary artery disease. Examples of frequently used class I agents are propafenone and flecainide. Class III drugs suppress reentry by prolonging refractory periods, but increase the likelihood of early afterdepolarisation and, therefore, torsades de pointes. Sotalol and amiodarone both produce class III effects, but these drugs also have beta blocking properties (class II effect). In addition, amiodarone displays class I and IV (calcium channel blocking) characteristics. Amiodarone will be discussed in detail in section 2 of this chapter.

Several new compounds developed for rhythm control are currently evaluated in clinical studies. Dronedarone, budiodarone and celivarone are amiodarone analogues with decreased lipophilicity and shorter elimination half-lifes (intended to reduce tissue accumulation and toxicity), while retaining amiodarone-like electrophysiological properties. ${ }^{95}$ Vernakalant and ranolazine are also multiple-ionchannel-blockers that predominantly inhibit atrium-specific currents ( $\left.I_{\mathrm{Kur}}, I_{\mathrm{Na}}\right)$, which may reduce the risk of ventricular arrhythmia. ${ }^{95}$

\subsubsection{Upstream Therapy}

Upstream therapies of AF include non-antiarrhythmic drugs that reduce structural remodeling caused by atrial inflammation and interstitial fibrosis. ${ }^{96}$ Therapies that alter the renin-angiotensin-aldosterone system (ACE inhibitors and angiotensin receptor blockers), as well as antiinflammatory and antioxidative drugs (statins, polyunsaturated fatty acids and glucocorticoids), are currently being investigated. Most of these drugs are relatively safe and readily available, and their present indications (hypertension, coronary artery disease, and heart failure) are among the most frequent causes of AF. Therefore, upstream therapy might not only be effective for secondary prevention, but also for primary prevention of AF. 


\subsection{Postoperative Atrial Fibrillation}

\subsubsection{Epidemiology}

Atrial fibrillation is a common complication of cardiac surgery, that increases patient mortality and morbidity, hospitalization duration, and healthcare resources. The incidence of postoperative atrial fibrillation (POAF) varies between $20 \%$ and $60 \%$, depending on definitions, types of surgical procedures, and methods of detection. ${ }^{97}$ The frequency of POAF is expected to rise in the future, due to increasing proportions of elderly cardiac surgical patients. The arrhythmia typically occurs within 2 to 4 days after the procedure.98,99 Many clinical variables are associated with the development of POAF, including advanced age, previous history of AF, male gender, heart failure, left atrial enlargement, valvular heart surgery, hypertension, chronic obstructive pulmonary disease, renal failure, diabetes mellitus, obesity, and cardiopulmonary bypass time..$^{100}$

\subsubsection{Mechanisms}

The mechanisms of AF in the postoperative patient are likely to be fundamentally similar to those in patients with AF who have not undergone surgery. In that setting, the electrophysiological basis of initiation and perpetuation of AF has already been discussed in detail in section 1.2. In the postoperative heart, multiple factors may alter atrial conduction and refractoriness, predisposing the patient to AF. ${ }^{101}$ These include trauma, elevation in atrial volume/pressure, use of inotropic agents, autonomic imbalance, and metabolic derangements. Recent studies focused on the role of inflammation and oxidative stress, and their molecular pathways in the pathogenesis of POAF. Based on these newly identified pathways, as for nonsurgery related $\mathrm{AF}$, upstream therapies for the prevention of POAF are currently being investigated. ${ }^{102}$

\subsubsection{Prevention and Treatment}

Besides managing comorbidities and correcting identifiable precipitants, the management strategy for POAF (as for AF in general) includes maintenance/restoration of sinus rhythm, prevention of rapid ventricular rates, and prevention of thromboembolism. Regarding prophylaxis of POAF, unless contraindicated, treatment with an oral beta blocker is recommended.9-11,103,104 Although therapy with sotalol (which features both beta blocking and class III antiarrhythmic effects) may be more effective, this drug is also associated with more side effects. ${ }^{99,105}$ In patients at high risk for POAF and in patients in whom beta blocker therapy is not possible, preoperative administation of amiodarone represents appropriate therapy. ${ }^{106}$ The routine prophylactic use of magnesium, nondihydropyridine calcium channel blockers, and digoxin is not supported by the current evidence. ${ }^{102,107}$ In addition to the use of an- 
tiarrhythmic drugs, upstream therapy with renin-angiotensin-aldosterone-system modulators, statins, polyunsaturated fatty acids, and glucocorticoids may be beneficial for prophylaxis of POAF. However, for these agents, there is a need for largescale clinical studies to determine their benefit. Apart from the here mentioned pharmacological therapies, biatrial pacing has been shown to decrease the occurence of POAF, but its complexity limits wide-scale application. ${ }^{101}$ Therefore generally pharmacological interventions are applied.

When POAF occurs, an AV nodal blocking agent, preferably a beta blocker unless contraindicated, should be used to achieve rate control in hemodynamically stable patients. Immediate electrical cardioversion has to be performed in hemodynamically unstable patients. If POAF does not spontaneously convert to sinus rhythm within 24 hours, rhythm control should be attempted, associated with antithrombotic medication. Amiodarone therapy is recommended in patients with depressed left ventricular function, whereas sotalol and class I antiarrhythmic drugs may be used in patients with preserved left ventricular function. 


\section{AMIODARONE}

Amiodarone was synthesized in 1962 in the pharmacological laboratory of Labaz in Belgium with the objective of treating angina pectoris and not for the control of cardiac arrhythmias. ${ }^{108,109}$ Chemists selected a natural product, khellin, which was isolated from the seeds of Ammi visnaga and was used in the Middle East as a diuretic and an antispasmodic. This molecule was then chemically modified in various steps to yield amiodarone, the structure of which is shown in figure 1 . As can be seen in this figure, amiodarone contains a butylated benzofuran moiety and a typical substituted diiodo phenoxy ring attached to an aliphatic tertiary amine group. Clinical trials showed that amiodarone was effective in reducing the number of anginal attacks, but it was also observed that amiodarone had antiarrhythmic properties. ${ }^{110}$ In 1970 Singh and Vaughan Williams described the drug's propensity to lengthen atrial and ventricular action potential duration as a function of time at a constant oral dose. This was suggested as the cornerstone of the concept of the class III antiarrhythmic action. ${ }^{111}$

As will be discussed in the next parts of this thesis, amiodarone proved to be an effective antiarrhythmic drug, although it also has significant drawbacks. Therefore, various analogues of amiodarone have been produced, some of which are currently clinically evaluated. However, these will not be discussed in detail.

\section{Amiodarone}
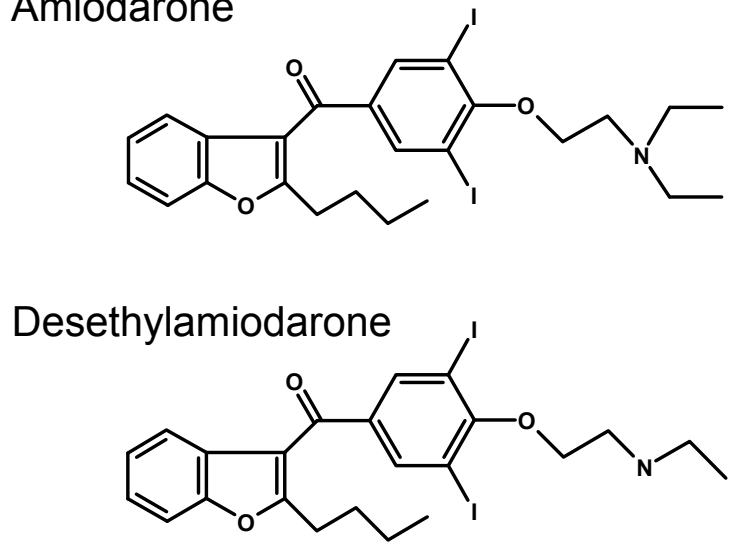

Figure 1. Chemical structures of amiodarone and desethylamiodarone.

\subsection{Clinical Use}

Amiodarone has been approved for use in many countries for management of a wide spectrum of cardiac arrhythmias, including $\mathrm{AF}$, atrial flutter, ventricular tachycardia, and ventricular fibrillation. A recent meta-analysis showed that in pa- 
tients at risk for sudden cardiac death, amiodarone reduces the incidences of sudden cardiac death and cardiovascular death by $29 \%$ and $18 \%$, respectively. ${ }^{112}$ However, amiodarone does not influence all-cause mortality, and bears the risk of causing pulmonary, thyroid and hepatic toxicity. Regarding management of AF, amiodarone has consistently been demonstrated to be superior to other antiarrhythmic drugs for maintenance of sinus rhythm. ${ }^{113-115}$ It is of particular value in patients with congestive heart failure developing $\mathrm{AF}$, in whom it virtually lacks negative inotropic effects, and increases left ventricular ejection fraction. ${ }^{116,117}$

\subsection{Pharmacokinetics}

Amiodarone is a highly lipophilic compound with a low total clearance and a large volume of distribution $(66 \mathrm{~L} / \mathrm{kg}) .{ }^{118}$ The major metabolite of amiodarone is desethylamiodarone (DEA, figure 1), which also produces electrophysiological effects. A recent study provided evidence of three more (minor) metabolites. ${ }^{119}$ Cytochrome $\mathrm{P}$ 450 3A4, present in liver and intestines, is mainly responsible for the $\mathrm{N}$ deethylation, leading to the formation of DEA. ${ }^{120}$ The bioavailability of oral amiodarone is variable $(30-80 \%)$, which may be attributed to some extent by interindividual variability in CYP3A4-mediated first-pass metabolism. During long-term therapy DEA reaches similar plasma concentrations as amiodarone. Both compounds show long elimination half-lives of typically 40-60 days, but in some individuals even of more than 100 days. Amiodarone and DEA are eliminated primarily via biliary excretion, whereas renal elimination is negligible.

During long-term therapy amiodarone and DEA attain high concentrations in many tissues, especially in fat, liver, and lung. ${ }^{121}$ Population studies showed that pharmacokinetics of amiodarone are best described by a typical two-compartment (plasma and tissue) model.122,123 However, the high lipophilicity and long half-life account for much confusion around amiodarone's clinical use. The effectiveness of an acute intravenous infusion rapidly disappears if the infusion is discontinued, since the drug leaves the serum with a distribution half-life of only 17 hours, and is sequestered in fat tissue. The opposite effect occurs if chronic amiodarone therapy is ceased; the rate of decline in serum concentrations is slowed by redistribution of amiodarone from fat tissue. In one study, a 25\% serum concentration decrement in 3 days and 50\% decrement in 36 days was observed after cessation of chronic amiodarone therapy. ${ }^{122}$ Steady-state amiodarone serum concentrations of 1.0 to $2.5 \mathrm{mg} / \mathrm{L}$ have been associated with antiarrhythmic effectiveness and acceptable toxicity. In myocardial tissue samples obtained from patients treated with chronic amiodarone therapy, amiodarone concentrations ranged from 12 to $147 \mathrm{mg} / \mathrm{kg}$. ${ }^{121}$ The latter also underlines the tendency of amiodarone to accumulate in tissues rather than to reside in blood plasma. 


\subsection{Electrophysiological Effects}

Although amiodarone was originally classified as a class III antiarrhythmic drug, it exhibits all 4 Vaughan Williams classes of action, i.e. sodium channel blockade, beta blockade, potassium channel blockade, and calcium channel blockade. ${ }^{93}$ The acute and chronic effects of amiodarone in the heart are substantially different, as reviewed by Kodama et al. ${ }^{124}$ Its main acute effect when administered intravenously is suppression of conductivity of the atrioventricular node with minimal effect on myocardial effective refractory periods (ERP). ${ }^{125}$ When administered orally for at least several weeks, amiodarone primarily lengthens action potential duration (APD) and ERP in most cardiac tissues. ${ }^{124}$ These differences cannot be explained in terms of pharmacokinetics. ${ }^{125}$ Both acute and chronic amiodarone prolong ERP more than APD because of development of postrepolarization refractoriness. ${ }^{126,127}$

Acute effects of amiodarone include attenuation of both inward and outward currents. ${ }^{124}$ Inward sodium and calcium currents are inhibited in a use- and voltagedependent manner, resulting in a decrease of the maximum upstroke velocity of the action potential, and decreased excitability and conductivity. Suppressed outward potassium currents include $I_{\mathrm{Kr}}, I_{\mathrm{K}, \mathrm{Ach}}, I_{\mathrm{K}, \mathrm{Na}}, I_{\mathrm{K} 1}$, and probably $I_{\mathrm{Ks}}$ and $I_{\mathrm{to}}$. The net effect of acute amiodarone on APD is unclear with prolongation, shortening, and no effect being reported in different studies. ${ }^{124}$

During chronic amiodarone therapy, its major metabolite DEA reaches similar plasma and tissue drug concentrations, and also produces significant electrophysiological effects. ${ }^{125}$ Chronic amiodarone treatment results in APD prolongation, which can be explained by decreased outward current densities ( $I_{\mathrm{K}}$ and $I_{\mathrm{to}}$ ). ${ }^{124}$ A recent study suggests that inhibitory effects of chronic amiodarone on both inward $\left(I_{\mathrm{Na}}\right)$ and outward $\left(I_{\mathrm{K}}\right)$ currents together produce a predominant prolongation of atrial APD which may explain its superior effectiveness to suppress AF. ${ }^{128}$ Furthermore chronic amiodarone produces electrophysiological changes that resemble those in the heart induced by hypothyroidism, possibly through interaction with thyroid hormone and its receptors. ${ }^{124}$

\subsection{Pleiotropic Effects}

Besides antiarrhythmic effects, amiodarone features an array of other therapeutic effects. Synthesized with the objective of treating angina pectoris, it is a potent vasodilator. ${ }^{129}$ Amiodarone rapidly accumulates in atherosclerotic vascular tissue, abolishing vascular autorhythmicity and improving endothelium-dependent function. It also exerts antioxidant activity and antiinflammatory activity by increasing levels of antioxidant system enzymes and inhibiting the generation of superoxide 
anion radicals. ${ }^{130,131}$ In addition, amiodarone produces antithrombotic effects such as impairment of thrombus formation and reduction of arterial tissue factor activity. ${ }^{132}$

Recent in vitro research demonstrated microbicidal activity of amiodarone against a broad range of species, including fungi, bacteria, protozoa, and viruses. ${ }^{133-}$ 136 Also, amiodarone could be used in the treatment of drug resistant tumor cells, because it is a potent multidrug resistance inhibitor. ${ }^{137}$ Finally, a therapeutic application might be Alzheimer's disease, since two key proteases in its pathogenesis, $\beta$ secretase and $\gamma$-secretase, are modulated by amiodarone. ${ }^{138}$

\subsection{Side Effects}

Amiodarone has few cardiac side effects, but frequently causes extracardiac side effects with a prevalence of $15 \%$ in the first year, increasing up to $50 \%$ during chronic therapy. In a meta-analysis of randomized placebo-controlled trials the amiodarone discontinuation rate was $31.6 \%$ versus $21.1 \%$ in the placebo group. ${ }^{112} \mathrm{~A}$ recent study showed that clinically relevant side effects of amiodarone are associated with long treatment durations and high cumulated doses, whereas the measured plasma levels of amiodarone or DEA as such are not correlated with side effects. ${ }^{123}$

The most frequent cardiac side effects of amiodarone are bradycardia and AV conduction disturbances (3-5\%), which are often dose-related and occur more frequently in elderly patients. On the other hand, amiodarone has a markedly lower potential to cause torsades de pointes compared to other class III antiarrhythmic drugs such as sotalol and dofetilide. A literature review reported that the incidence of torsades de pointes was $0.7 \%$ in 2878 patients included in 17 uncontrolled studies, while no proarrhythmic effects were observed in 1464 patients treated in 7 controlled studies. ${ }^{139}$

Amiodarone's most frequent extracardiac side effects include corneal microdeposits (>90\%), photosensitivity (25-75\%), hepatotoxicity $(15-30 \%)$, hyperthyroidism and hypothyroidism (4-20\%), tremor and ataxia (3-35\%), blue-gray skin discoloration $(4-9 \%)$, and pulmonary toxicity $(3-9 \%) .112,140-142$ The most serious side effect is pulmonary fibrosis, which occurs in $<3 \%$ of patients. Regarding the mechanisms of amiodarone toxicity, thyroid dysfunction is in part related to its high iodine content ( $37 \%$ by weight), while ultrastructural changes typical of a lipidosis have been observed in skin, lung, and liver. ${ }^{121}$ 


\section{INTRAPERICARDIAL AND EPICARDIAL PHARMACOTHERAPY}

Applying a drug locally may enhance its effectiveness and lower the required dose. As a result, the systemic concentration of the drug and hence systemic side effects may be minimized. The pericardial cavity is a natural reservoir surrounding almost the entire heart, and is ideally suited for local cardiac drug delivery since it can be filled with drug solutions and provides space for epicardial application of drug delivery systems. ${ }^{143}$ Already in 1936 it was shown that pericardial delivery of local anesthetics reduces the excitability of the heart and may terminate arrhythmias during cardiac surgery. ${ }^{144}$ Nevertheless, until two decades ago, research on pericardial drug therapy mainly focused on the treatment of pericardial disease (see section 3.4.2.). This was because of a lack of minimally-invasive techniques to access the non-effusive pericardial cavity. ${ }^{145}$ In recent years rapid advances have been made in the field of pericardial access and drug delivery systems. ${ }^{143}$ Peri- and epicardial drug therapy could therefore not only be a promising way to treat pericardial disease, but also offers new therapeutic strategies to counter heart diseases such as arrhythmias, coronary artery disease and myocardial disease. In combination with new treatment modalities such as stem cell therapy and gene therapy, periand epicardial drug delivery may become of increasing importance in the treatment of cardiac disease.

\subsection{Pericardial Physiology}

The pericardial cavity is delineated by the mesothelium of the visceral pericardium and the mesothelium of the parietal pericardium. Visceral pericardium is also known as epicardium. The cavity normally contains $15-35 \mathrm{ml}$ of fluid, which facilitates heart movement. ${ }^{146}$ It is in essence a plasma ultrafiltrate with accumulated products of the mesothelium and myocardium. These products have paracrine activity influencing coronary and myocardial physiology. ${ }^{146}$ There is continuous mixing of fluid throughout the pericardial cavity, so that fluid content is homogeneously distributed. ${ }^{147-149}$ In addition, the produced pericardial fluid is cleared from the pericardial space mainly by lymphatic drainage, accounting for a clearance of about $18 \%$ of the total pericardial volume per hour in sheep. ${ }^{150}$ However, in the case of intrapericardial hemorrhage as much as $150 \mathrm{ml}$ of fluid may cause cardiac tamponade. Moreover, slow accumulation of fluid, for example as a result of pericardial inflammation or malignancy, may lead to volumes of $2 \mathrm{~L}$ before cardiac decompensation occurs. ${ }^{151}$ 


\subsection{Modulating the Peri- and Epicardial Paracrine Function}

Several animal studies have been performed to investigate and modulate the intrapericardial (IPC) release of specific substances by the pericardial mesothelium and myocardium. Release of prostacyclin can be stimulated by IPC delivered arachidonic acid and inhibited by indomethacine. ${ }^{152}$ An increase in the concentration of prostaglandins in the pericardial fluid inhibits efferent sympathetic cardiac nerve effects. This mechanism can be used to prevent reperfusion-induced ventricular fibrillation. ${ }^{153}$ Endothelin-1 (ET-1) is an endogenous vasoconstrictor and is also present in the pericardial fluid. ${ }^{154}$ Increased IPC ET-1 induces myocardial ischemia and ventricular tachycardia. Administering an intravenous ET-1 antagonist prevents ventricular tachycardia but not IPC ET-1 induced ischemia. ${ }^{155}$ Intrapericardially delivered catecholamines and adenine nucleosides result in an increase of IPC ET-1. Their interactions and effects on coronary vasotonus are currently investigated. ${ }^{156-158}$

Many growth factors, including angiogenic factors such as fibroblast growth factors (FGF), are present at high concentrations in the pericardial fluid. These may accumulate in various types of heart disease, but the physiological or pathological role of growth factors in the pericardial fluid so far has not been thoroughly investigated. ${ }^{159}$ However, several studies investigated IPC delivery of FGF to stimulate angiogenesis in the treatment of ischemic heart disease. These studies showed improvement of the cardiac perfusion and capillarization of the heart under ischemic conditions, which was more effective than systemically-applied FGF, and was accompanied by higher cardiac tissue levels of FGF in case of IPC administration. ${ }^{160-164}$

\subsection{Percutaneous Pericardial Access}

Recently, percutaneous access has been made feasible not only into the significantly-effusive pericardial cavity, but also into the non-effusive pericardium. Both epicardial (transthoracic) and endocardial (transmyocardial) approaches have been demonstrated. These techniques avoid the pleural space and can be performed minimally-invasively, which may enable ambulatory diagnostic and therapeutic epicardial interventions. In addition to delivery of drugs, stem cells and adenoviruses for gene transfer, other promising epicardial interventions have been reported, such as endoscopy, echocardiography, and electrophysiological mapping, pacing and ablation. ${ }^{165-168}$ To obviate immobilization of the heart and reduce access limitations, mobile robotic devices for navigation and intervention on the beating heart are currently being developed. ${ }^{169,170}$ 


\subsubsection{Transthoracic Approach}

The most frequently used method is the subxiphoid technique where entry to the pericardial cavity occurs close to the apex of the right ventricle. A needle-based method was described in 1996, using a Tuohy introducer and fluroscopic guidance. ${ }^{167}$ Medium contrast is injected to demonstrate the intrapericardial position of the needle tip. However, this method is associated with a considerable risk of hemopericardium, and its applicability is limited in patients with prior cardiac surgery. ${ }^{171}$ To enhance safety of transthoracic access into the non-effusive pericardial space, a novel instrument, the PerDUCER, was introduced in 1998. This technique involves manual suction with a syringe for capturing pericardium in a hemispherical cavity of the device, followed by tangential needle puncture. ${ }^{145,172}$ Although no major complications were reported, pericardial capture was frequently hampered by pericardial fat and pericardial thickening, requiring thoracoscopic assistance. ${ }^{173,174}$ Recently, an improved tool, the AttachLifter, has been described, employing flexible tissue clamps, a needle separate from the vacuum channel, and a safety ridge. ${ }^{175}$ These features prevent injury to the epicardium and enable access even through markedly thickened pericardium.

\subsubsection{Transmyocardial Approach}

Two decades ago, needle insertion through the right atrial wall into the pericardial cavity was frequently observed during transseptal catheterization attempts. Although it was an unwanted course, serious complications did not occur. Its potential was recognized in 1995, when transatrial puncture was deliberately employed for IPC injection of growth factors. ${ }^{160}$ The feasibility and safety of this technique was demonstrated in two animal models. ${ }^{176,177}$ In another pre-clinical study, pericardial access was achieved through helical puncture of the right ventricular apex without complications. ${ }^{178}$ The transmyocardial approach does not require general anesthesia and does not breach the pericardial wall. Despite these advantages, this method carries the risk of myocardial rupture, and does not seem suitable for long-term pericardial catheterization.

\subsubsection{Chronic Pericardial Access}

Chronic intrapericardial catheterization would allow continuous controlled delivery of therapeutic agents. This is especially desirable for drugs with a narrow therapeutic width, and for diseases requiring frequent bolus doses, such as paroxysmal arrhythmias. Scarce data exist with respect to long-term tolerability of foreign material, such as a catheter, in the pericardial cavity. In a study in five pigs in which an IPC catheter had been implanted for a total of 6 months, histology showed moderate, albeit probably clinically significant, inflammatory infiltration and fibrosis adjacent to the catheter. ${ }^{179}$ In another study catheters were implanted in the pericardium 
of seven dogs, and remained bidirectionally patent for more than a year. ${ }^{180}$ Six animals were sacrificed after an average of 213 days, and adhesion tissue was found only at the site of catheter entry through the pericardium, with essentially no chronic inflammation in any of the pericardial sections away from the tract of the catheter. Thus far clinical experience with chronic IPC catheterization in patients has not been reported. Promising alternatives for chronic percutaneous access are fully implantable drug pump systems which require only intermittent refilling, or epicardial application of drug delivery systems which is discussed in detail separately.

\subsection{Intrapericardial Injections or Infusions of Drug Solutions}

\subsubsection{Pharmacokinetic Considerations}

Since the pericardial space is a closed compartment and is filled with fluid surrounding the heart, it seems an ideal compartment for locally applying drugs aiming to treat the diseased heart. This is substantiated by a magnetic resonance imaging study which showed that IPC administered agents are sufficiently mixed within pericardial fluid to penetrate the epicardial surface of the heart uniformly. ${ }^{181} \mathrm{How}$ ever, it should be realized that endocardial layers are less well reached, producing transmural concentration gradients in both ventricles and atria. ${ }^{182-188}$

That there is a pharmacokinetic advantage of acute and chronic IPC delivery has been demonstrated for instance for a variety of substances with molecular weights ranging from $348 \mathrm{Da}$ to $155 \mathrm{kDa}$, without a clear relationship between molecular size and magnitude of the obtained advantage. ${ }^{189}$ The local advantages (resulting in high local versus low systemic concentrations) of IPC applied agents can be mainly explained by their low clearances from the pericardial space compared with the relatively high plasma clearances. Movement of agents between the pericardial fluid and the epicardium is not unidirectional, as was shown for these substances as well as for intravenously administered procainamide which rapidly diffused into the pericardial space. ${ }^{149,189}$

\subsubsection{Treatment of Pericarditis: a Clinical Application of Intrapericardial Drug Delivery}

IPC drug delivery, as attractive as it seems, so far is mostly applied in non-clinical settings, but there are some exceptions. IPC drug delivery has been performed for decades in patients with neoplastic and uremic pericarditis, and to the present day, pericarditis is the only indication for which IPC pharmacotherapy is mentioned in international guidelines. ${ }^{190}$ Most experience has been obtained with corticosteroids, particularly with triamcinolone. Already in 1978 the effectiveness and safety of treatment in forty-five patients with intractable uremic pericardial effusion was reported. ${ }^{191}$ More recently, IPC delivery of triamcinolone was shown beneficial in 
eighty-four patients with autoreactive pericarditis. ${ }^{192}$ Regarding bacterial pericarditis, the primary focus of IPC pharmacotherapy has been prevention of constrictive pericarditis through instillation of fibrinolytics, such as urokinase and streptokinase. ${ }^{193-195}$ Theoretically, IPC delivery of antibiotics could be very effective in treating bacterial pericarditis, but thus far, only one preclinical study has been reported. ${ }^{196}$ A final category of pericarditis eligible for IPC pharmacotherapy is neoplastic pericarditis. Both cytostatic and sclerosing agents may be administered into the pericardial cavity to control malignant effusions. ${ }^{190}$ Substantial clinical experience has been obtained with cisplatin, thiotepa, bleomycin, and tetracycline. ${ }^{197-205}$

\subsubsection{Intrapericardial Application of Antiarrhythmic Drugs}

The electrophysiological effects of intrapericardially delivered antiarrhythmic drugs were studied for the first time in $1936 .{ }^{144}$ Injections of metycaine, cocaine and procaine solutions were all found to affect epicardial monophasic action currents but had virtually no effect on mean blood pressure. In addition, the minimal stimulus necessary to produce an extrasystole was increased. IPC procaine completely abolished peripheral vagus effects on heart rate and blood pressure. These findings were applied in experimental heart surgery to slow tachycardia and to stop atrial fibrillation during an operation. ${ }^{144}$ Five decades later, effects of IPC procaine on cardiac afferents and efferents were explored more elaborately in several animal studies. ${ }^{206-210}$

IPC administration of beta-adrenoceptor and muscarinic cholinergic receptor antagonists, reported in 1987, produced the same effects on heart rate as did conventional higher intravenous doses of the drugs. ${ }^{211}$ Delivery into the pericardial sac had little effect on ventricular contractility, and plasma levels were significantly less than after the usual (higher) intravenous dose. Another study with the hydrophylic beta blocker esmolol showed prolonged suppression of sinus tachycardia without a decrease of contractility or blood pressure upon IPC administration, while effective tachycardia suppression of intravenously applied esmolol could only be achieved at doses that also reduced contractility and blood pressure. ${ }^{212}$ Pharmacokinetic advantages were also demonstrated during chronic IPC delivery of sotalol and atenolol with similar antitachycardiac effects at ten- to thirty-fold lower dose compared with intravenous delivery. ${ }^{213}$

In the past two decades research focus shifted to treatment of atrial fibrillation through IPC delivery of antiarrhythmic drugs, including digoxin, amiodarone, procainamide, ibutilide, flecainide, and ranolazine. ${ }^{149,179,184,186-188,214,215}$ In these studies drug instillation into the pericardial cavity produced significant atrial electrophysiological effects. Global ventricular electrophysiology was not affected in all but one study; IPC infusion of flecainide in goats resulted in marked ST segment elevation. ${ }^{188}$ This raises the concern that a transmural concentration gradient of antiar- 
rhythmic drugs could increase the vulnerability to ventricular arrhythmias. The same could hold true for vulnerability to atrial arrhythmias, which might explain that in two studies IPC delivery did not result in higher AF termination rates compared with control. ${ }^{188,214}$

\subsubsection{Intrapericardial Nitric Oxide Donors for Treatment of Ischemia-Induced Arrhythmias}

Due to their epicardial course, coronary arteries are eligible targets for IPC drug delivery. Intravenous infusion of nitric oxide donors has been demonstrated to attenuate ischemic injury and arrhythmias, but its use is limited by a relatively short duration of action and systemic adverse effects such as hypotension. These limitations may be circumvented by epicardial pharmacotherapy. Indeed, IPC delivery of nitroprusside more effectively decreased platelet aggregation and cyclic flow variation in endothelium-injured coronary arteries. ${ }^{216}$ In addition, instillation of Larginine into the pericardial cavity reduced severity of ventricular arrhythmias during sympathetic stimulation in acute coronary occlusion. ${ }^{217}$ Furthermore, it was shown that sustained coronary vasodilation and suppression of ventricular fibrillation could be achieved with IPC delivery of nitroglycerin, whereas systemic hypotension did not occur. ${ }^{218,219}$

\subsection{Epicardial Application of Drug Delivery Systems}

IPC delivery of drug solutions is suitable for short-term pharmacotherapy, however chronic infusion is clinically less feasible because it requires prolonged pericardial access with an increased risk of catheter-associated complications such as infection. ${ }^{179,180}$ Epicardial application of drug delivery systems may enable controlled drug delivery that is sustained and localized without the need for chronic percutaneous access. Additional polymeric layers can be used to achieve pulsatile drug release or, in case of a backing layer, unidirectional drug release. ${ }^{220}$ Furthermore, drug delivery systems may serve as tissue-forming cell/scaffold constructs for tissue engineering of cardiovascular structures..21-223 ${ }^{2}$ Moreover, drug-releasing matrices form a barrier, which might be applied during cardiac surgery to enable epicardial hemostasis and to prevent pericardial adhesions. ${ }^{224}$

Both natural and synthetic biomaterials have been investigated for local drug delivery. Frequently used natural materials include collagen, fibrin, hyaluronic acid, and decellularized tissues. In the past decades new classes of synthetic polymer classes for drug delivery have been developed such as polyesters, polyanhydrides, polyorthoesters and polycarbonates. ${ }^{225}$ Both biocompatibility and biodegradability are of paramount importance for the development of these materials. Drug release from biodegradable matrices is not only determined by diffusion, but also by the 
rate of degradation. ${ }^{226}$ Furthermore, polymeric materials can be tailored to achieve stimulus-responsive release characteristics, where responsiveness may come from changes in e.g. temperature, or $\mathrm{pH}$, or can be externally triggered e.g. by applying ultrasound. ${ }^{220}$ Drug delivery can also be electronically assisted in situ using technologies such as iontophoresis on matrices applied to pacing electrodes. ${ }^{183}$

\subsubsection{Corticosteroids: an Example of Clinically Applied Epicardial Pharmacotherapy}

Perhaps the most extensive clinical experience with epicardial application of drug delivery systems has been obtained with steroid-eluting pacing leads.227 Steroid elution not only attenuates the initial inflammation after lead implantation, but also reduces stimulation thresholds over time. Effective drug concentrations in the local area of the electrode can probably be maintained for several years. ${ }^{228}$ Another promising use of steroid-releasing matrices is the prevention of pericardial adhesions. The effectiveness of dexamethasone-loaded biodegradable poly(lactide-coethyleneglycol) films has been demonstrated in a rabbit model of postoperative cardiac adhesions. ${ }^{229}$ Because inflammation plays a significant role in the pathophysiology of postoperative atrial fibrillation, epicardial delivery of steroids might also provide protection against this complication. Indeed, a mixture of triamcinolone and fibrin glue reduced postoperative atrial tachyarrhythmias in the sterile pericarditis model, while tensile strength of the healing atriotomy was maintained and plasma steroid levels were low. 230

\subsubsection{Epicardial Application of Antiarrhythmic Drugs}

The release of antiarrhythmic drugs from epicardial drug delivery systems was first investigated in acute experiments with non-biodegradable polyurethane patches. Lidocaine-eluting matrices were placed on the left ventricular epicardium of dogs to treat ouabain-induced and rapid pacing-induced ventricular tachycardia. ${ }^{231,232}$ Ventricular contractile performance was minimally attenuated at the site of matrix placement, and peripheral plasma lidocaine levels were approximately ten times lower than coronary venous levels. In another study, effects of epicardial delivery of propranolol, verapamil, and sotalol on prevention of ischemic ventricular arrhythmias were also investigated in anesthesized dogs by use of polyurethane matrices. ${ }^{182,185,233}$ Although local therapeutic drug concentrations were achieved with all the three drugs, epicardial propranolol was not found to be effective, and epicardial sotalol was only effective when administered in the ischemic zone.

In 1994 long-term epicardial drug delivery with three different controlledrelease formulations of sotalol was reported. ${ }^{234}$ Polyurethane and silicone matrices, and biodegradable poly(lactide-co-glycolide) microspheres were administered in seven-day, sixty-day, and thirty-day dog experiments, respectively. In these chronic-release studies, while using a significantly lower total dose, myocardial drug 
concentrations were comparable to those observed during short-term intravenous infusion. Transmural ventricular sotalol concentration gradients (with higher epicardial than endocardial drug levels) were reduced as the duration of experiments was increased.

The feasibility of iontophoretic epicardial delivery of antiarrhythmic drugs has been demonstrated in two studies. In a canine model of myocardial infarction, procainamide was administered via an implantable defibrillator patch electrode that was modified to contain a drug reservoir. ${ }^{183}$ Iontophoretic delivery rapidly suppressed ventricular tachycardia, and produced higher myocardial drug concentrations than either passive diffusion or intravenous infusion. In another study in dogs, an epicardial drug reservoir implant configured with a rate-limiting heterogeneous cation-exchange membrane showed sotalol release rates that were responsive to current modulation. ${ }^{235}$ This resulted in regional enhancement of sotalol levels and current-dependent changes in ventricular effective refractory periods.

\subsubsection{Gene Therapy via the Epicardium in Order to Treat Atrial Fibrillation}

Two studies investigated feasibility of atrial gene transfer via epicardial application of poloxamer hydrogels. Release of adenoviral vectors to the epicardial surface of porcine atria resulted in complete transmural atrial gene transfer of a dominantnegative mutant of the $I_{\mathrm{Kr}}$ potassium channel. ${ }^{236}$ This eliminated burst-pacing induced persistent AF by prolonging atrial action potential duration and refractory period, whereas ventricular electrophysiology was not affected.237 A mechanism involving antiviral antibody tethering was used to produce adenovirus-releasing collagen-coated polyurethane patches. ${ }^{238}$ Seven days after epicardial application to right atria of pigs, significant localized transduction was observed with no evidence of gene expression beyond the margins of the implant sites. Gene transfer was also noted in lung tissue, likely due to coronary venous uptake of nonlocalized adenoviral viral vector, whereas no transduction was detected in other organs. By contrast, epicardial direct injections of viral vectors produced sparse, needle-track-oriented gene expression patterns. Based on the above text, it can be concluded that the feasibility of epicardially delivered gene therapy - be it or not in relation to antiarrhythmic treatment - still awaits further investigation and optimization. 


\section{Aims AND OUtLINE OF THE THESIS}

Pharmacotherapy of cardiac disease is often accompanied by systemic (non-cardiac) side effects. This also holds true for antiarrhythmic treatment of atrial fibrillation (AF), the most common cardiac arrhythmia requiring medical attention. The present thesis explores local cardiac pharmacotherapy via intrapericardial (IPC) and epicardial drug delivery in order to enable, or to possibly enhance, antiarrhythmic effectiveness, while minimizing systemic adverse effects.

Amiodarone, a highly lipophilic multi-ion channel-blocking antiarrhythmic drug, was selected for further studies. It is the most frequently used agent worldwide for maintaining sinus rhythm in patients with AF, featuring superior effectiveness combined with a low potential for causing torsades de pointes. ${ }^{109}$ The fact that amiodarone therapy is also associated with numerous systemic side effects, renders it a clinically relevant candidate to test the concept of local drug delivery.

Three approaches for epicardial amiodarone release were investigated in preclinical experiments, including IPC-infused amiodarone solutions, epicardially sprayed in-situ produced hydrogels in which amiodarone is included, and epicardially sutured bilayered amiodarone loaded patches. These approaches were expected to produce different amiodarone distribution patterns, each associated with a specific profile of electrophysiological effects, effect-duration, and risk of ventricular and systemic side effects.

From pharmacological and electrophysiological perspectives, one of the most unique and interesting aspects of epicardial drug delivery is the resulting transmural myocardial concentration gradient. For example, previous work from our group showed that IPC drug delivery produces steep transmural concentration gradients in the atria. ${ }^{188}$ This necessitates meticulous measurements of both epicardial and endocardial drug concentrations and electrophysiological parameters. Regional dispersion of electrophysiological properties may increase vulnerability to both ventricular and atrial arrhythmias.

Chapter 2 describes a novel method for the determination of amiodarone and dronedarone, and their principal metabolites in plasma and myocardium by highperformance liquid chromatography and UV-detection. Dronedarone, a noniodinated benzofuran analogue of amiodarone, has recently been regulatory approved for maintenance of sinus rhythm in patients with AF. ${ }^{239}$ In view of the current thesis, this newly-developed method enables sensitive and specific measurement of amiodarone concentrations in plasma, pericardial fluid, and tissue samples. Because it only required a small sample-quantity, and calibration curves were linear in a wide range of amiodarone concentrations, the technique was found to be well suited for measurement of transmural myocardial drug concentration gradients. 
Chapter 3 compares drug distribution and electrophysiological effects during IPC and intravenous delivery of amiodarone and sotalol in goats. Because both drugs differ in molecular weight, hydrophilicity, and electrical charge, one would expect differences in transmural myocardial drug distribution and plasma levels during IPC delivery, resulting in differences between epicardial and endocardial electrophysiological effects, and vulnerability to arrhythmias. It is shown that IPC delivery of amiodarone and sotalol increases atrial drug concentrations and electrophysiological effects at reduced plasma drug concentrations. These potential benefits are particularly prominent for IPC-delivered amiodarone.

Chapter 4 explores the feasibility of prolonged and localized epicardial amiodarone-release via an in-situ produced biodegradable poly(ethylene glycol)-based hydrogel. The gel matrix itself is already being used for prevention of pericardial adhesions after cardiac surgery. In experiments with goats the two-component polymer system was sprayed onto the epicardial surface of the right atrium. The hypothesis was tested that the amiodarone-loaded hydrogel would enable sustained suppression of burst-pacing-induced atrial tachyarrhythmias, while systemic drug levels remained low.

Chapter 5 addresses the question whether a better atrium-specific drug delivery could be achieved by using a bilayered patch with an amiodarone loaded matrix facing the epicardium and an impermeable backing layer facing the pericardium. The latter was designed to minimize drug release into the pericardial fluid, thereby slowing distribution to other cardiac regions and enabling longer-lasting sitespecific drug delivery. The poly(ethylene glycol)-based drug-loaded matrix degrades within several weeks, whereas the poly(lactide-co-caprolactone)-based backing layer degrades much slower, on the order of 6 to 12 months, ensuring preserved impermeability during amiodarone release. Prove of concept experiments were conducted in goats to evaluate drug distribution and electrophysiological effects.

Chapter 6 focuses on epicardial delivery of amiodarone in a goat-model of burst-pacing-induced persistent AF. Simultaneous epicardial and endocardial electrophysiological measurements were performed to evaluate the effects of transmural myocardial amiodarone gradients on atrial electrophysiology. This study addressed the hypothesis that epicardial application of an amiodarone-releasing hydrogel attenuates stability of AF-paroxysms in remodeled atria.

Chapter 7 offers a general discussion of the main findings of this thesis, integrates these into a broader scientific perspective, and touches upon possible implications for future research and clinical management. 


\section{REFERENCES}

1. Cushny AR, Edmunds CW. Paroxysmal irregularity of the heart and auricular fibrillation. In: Bulloch W, ed. Studies in pathology. Aberdeen, Scotland: University of Aberdeen. 1906:95-110.

2. Einthoven W. Le télécardiogramme. Arch Int Physiol. 1906;4:132-164.

3. Mackenzie J. The cause of heart irregularity in influenza. BMJ. 1902;1:1411-1413.

4. Fye WB. Tracing atrial fibrillation-100 years. N Engl J Med. 2006;355(14):1412-1414.

5. Rothberger C, Winterberg H. Vorhofflimmern und arrhythmia perpetua. Wien Klin Wochenschr. 1909;22:839-844.

6. Lewis T. Auricular fibrillation: a common clinical condition. BMJ. 1909;2:1528.

7. Nattel S. New ideas about atrial fibrillation 50 years on. Nature. 2002;415(6868):219-226.

8. Prystowsky EN, Katz AM. Atrial Fibrillation. In: Textbook of Cardiovascular Medicine. Philadelphia: Lippincott-Raven. 1998:1661.

9. Fuster V, Ryden LE, Cannom DS, et al. ACC/AHA/ESC 2006 Guidelines for the Management of Patients with Atrial Fibrillation: a report of the American College of Cardiology/American Heart Association Task Force on Practice Guidelines and the European Society of Cardiology Committee for Practice Guidelines (Writing Committee to Revise the 2001 Guidelines for the Management of Patients With Atrial Fibrillation): developed in collaboration with the European Heart Rhythm Association and the Heart Rhythm Society. Circulation. 2006;114(7):e257354.

10. Camm AJ, Kirchhof P, Lip GY, et al. Guidelines for the management of atrial fibrillation: the Task Force for the Management of Atrial Fibrillation of the European Society of Cardiology (ESC). Eur Heart J. 2010;31(19):2369-2429.

11. Fuster V, Ryden LE, Cannom DS, et al. ACCF/AHA/HRS 2011 focused updates incorporated into the ACC/AHA/ESC 2006 guidelines for the management of patients with atrial fibrillation: a report of the American College of Cardiology Foundation/American Heart Association Task Force on practice guidelines. Circulation. 2011;123(10):e269-367.

12. Kopecky SL, Gersh BJ, McGoon MD, et al. The natural history of lone atrial fibrillation. A population-based study over three decades. N Engl J Med. 1987;317(11):669-674.

13. Benjamin EJ, Levy D, Vaziri SM, et al. Independent risk factors for atrial fibrillation in a population-based cohort. The Framingham Heart Study. JAMA. 1994;271(11):840-844.

14. Feinberg WM, Blackshear JL, Laupacis A, et al. Prevalence, age distribution, and gender of patients with atrial fibrillation. Analysis and implications. Arch Intern Med. 1995;155(5):469-473.

15. Go AS, Hylek EM, Phillips KA, et al. Prevalence of diagnosed atrial fibrillation in adults: national implications for rhythm management and stroke prevention: the AnTicoagulation and Risk Factors in Atrial Fibrillation (ATRIA) Study. JAMA. 2001;285(18):2370-2375.

16. Krahn AD, Manfreda J, Tate RB, et al. The natural history of atrial fibrillation: incidence, risk factors, and prognosis in the Manitoba Follow-Up Study. Am J Med. 1995;98(5):476-484.

17. Friberg J, Buch $\mathrm{P}$, Scharling $\mathrm{H}$, et al. Rising rates of hospital admissions for atrial fibrillation. Epidemiology. 2003;14(6):666-672.

18. Wolf PA, Abbott RD, Kannel WB. Atrial fibrillation as an independent risk factor for stroke: the Framingham Study. Stroke. 1991;22(8):983-988.

19. Atrial Fibrillation Investigators. Risk factors for stroke and efficacy of antithrombotic therapy in atrial fibrillation. Analysis of pooled data from five randomized controlled trials. Arch Intern Med. 1994;154(13):1449-1457. 
20. Stewart S, Hart CL, Hole DJ, et al. A population-based study of the long-term risks associated with atrial fibrillation: 20-year follow-up of the Renfrew/Paisley study. Am J Med. 2002;113(5):359-364.

21. Vidaillet H, Granada JF, Chyou PH, et al. A population-based study of mortality among patients with atrial fibrillation or flutter. Am J Med. 2002;113(5):365-370.

22. Chugh SS, Blackshear JL, Shen WK, et al. Epidemiology and natural history of atrial fibrillation: clinical implications. J Am Coll Cardiol. 2001;37(2):371-378.

23. Levy S, Maarek M, Coumel P, et al. Characterization of different subsets of atrial fibrillation in general practice in France: the ALFA study. The College of French Cardiologists. Circulation. 1999;99(23):3028-3035.

24. Spodick DH. Arrhythmias during acute pericarditis. A prospective study of 100 consecutive cases. JAMA. 1976;235(1):39-41.

25. Morgera T, Di Lenarda A, Dreas L, et al. Electrocardiography of myocarditis revisited: clinical and prognostic significance of electrocardiographic changes. Am Heart J. 1992;124(2):455-467.

26. Hamer ME, Wilkinson WE, Clair WK, et al. Incidence of symptomatic atrial fibrillation in patients with paroxysmal supraventricular tachycardia. J Am Coll Cardiol. 1995;25(5):984-988.

27. Diker E, Aydogdu S, Ozdemir M, et al. Prevalence and predictors of atrial fibrillation in rheumatic valvular heart disease. Am J Cardiol. 1996;77(1):96-98.

28. Wang TJ, Larson MG, Levy D, et al. Temporal relations of atrial fibrillation and congestive heart failure and their joint influence on mortality: the Framingham Heart Study. Circulation. 2003;107(23):2920-2925.

29. Liberthson RR, Salisbury KW, Hutter AM, Jr., et al. Atrial tachyarrhythmias in acute myocardial infarction. Am J Med. 1976;60(7):956-960.

30. Cameron A, Schwartz MJ, Kronmal RA, et al. Prevalence and significance of atrial fibrillation in coronary artery disease (CASS Registry). Am J Cardiol. 1988;61(10):714-717.

31. Robinson K, Frenneaux MP, Stockins B, et al. Atrial fibrillation in hypertrophic cardiomyopathy: a longitudinal study. J Am Coll Cardiol. 1990;15(6):1279-1285.

32. Tikoff G, Schmidt AM, Hecht HH. Atrial fibrillation in atrial septal defect. Arch Intern Med. 1968;121(5):402-405.

33. Goldhaber SZ, Visani L, De Rosa M. Acute pulmonary embolism: clinical outcomes in the International Cooperative Pulmonary Embolism Registry (ICOPER). Lancet. 1999;353(9162): 1386-1389.

34. Buch P, Friberg J, Scharling H, et al. Reduced lung function and risk of atrial fibrillation in the Copenhagen City Heart Study. Eur Respir J. 2003;21(6):1012-1016.

35. Frost L, Vestergaard P, Mosekilde L. Hyperthyroidism and risk of atrial fibrillation or flutter: a population-based study. Arch Intern Med. 2004;164(15):1675-1678.

36. Gami AS, Pressman G, Caples SM, et al. Association of atrial fibrillation and obstructive sleep apnea. Circulation. 2004;110(4):364-367.

37. Frost L, Hune LJ, Vestergaard P. Overweight and obesity as risk factors for atrial fibrillation or flutter: the Danish Diet, Cancer, and Health Study. Am J Med. 2005;118(5):489-495.

38. Ettinger PO, Wu CF, De La Cruz C, Jr., et al. Arrhythmias and the "Holiday Heart": alcoholassociated cardiac rhythm disorders. Am Heart J. 1978;95(5):555-562.

39. Spies C, Trohman RG. Narrative review: Electrocution and life-threatening electrical injuries. Ann Intern Med. 2006;145(7):531-537.

40. Maisel WH, Rawn JD, Stevenson WG. Atrial fibrillation after cardiac surgery. Ann Intern Med. 2001;135(12):1061-1073. 
41. Aviles RJ, Martin DO, Apperson-Hansen C, et al. Inflammation as a risk factor for atrial fibrillation. Circulation. 2003;108(24):3006-3010.

42. Coumel P. Autonomic influences in atrial tachyarrhythmias. J Cardiovasc Electrophysiol. 1996;7(10):999-1007.

43. Arnar DO, Thorvaldsson S, Manolio TA, et al. Familial aggregation of atrial fibrillation in Iceland. Eur Heart J. 2006;27(6):708-712.

44. Page RL, Wilkinson WE, Clair WK, et al. Asymptomatic arrhythmias in patients with symptomatic paroxysmal atrial fibrillation and paroxysmal supraventricular tachycardia. Circulation. 1994;89(1):224-227.

45. Gallagher MM, Guo XH, Poloniecki JD, et al. Initial energy setting, outcome and efficiency in direct current cardioversion of atrial fibrillation and flutter. J Am Coll Cardiol. 2001;38(5):14981504.

46. Wittkampf $\mathrm{FH}$, de Jongste $\mathrm{MJ}$, Lie $\mathrm{HI}$, et al. Effect of right ventricular pacing on ventricular rhythm during atrial fibrillation. J Am Coll Cardiol. 1988;11(3):539-545.

47. Andersen HR, Nielsen JC, Thomsen PE, et al. Long-term follow-up of patients from a randomised trial of atrial versus ventricular pacing for sick-sinus syndrome. Lancet. 1997;350(9086):1210-1216.

48. Levy S, Ricard P, Lau CP, et al. Multicenter low energy transvenous atrial defibrillation (XAD) trial results in different subsets of atrial fibrillation. J Am Coll Cardiol. 1997;29(4):750-755.

49. Damiano RJ, Jr., Gaynor SL, Bailey M, et al. The long-term outcome of patients with coronary disease and atrial fibrillation undergoing the Cox maze procedure. J Thorac Cardiovasc Surg. 2003;126(6):2016-2021.

50. Gillinov AM, McCarthy PM. Advances in the surgical treatment of atrial fibrillation. Cardiol Clin. 2004;22(1):147-157.

51. Haissaguerre M, Jais $\mathrm{P}$, Shah DC, et al. Spontaneous initiation of atrial fibrillation by ectopic beats originating in the pulmonary veins. N Engl J Med. 1998;339(10):659-666.

52. Wijffels MC, Kirchhof CJ, Dorland R, et al. Atrial fibrillation begets atrial fibrillation. A study in awake chronically instrumented goats. Circulation. 1995;92(7):1954-1968.

53. Garrey WE. Auricular fibrillation. Physiol Rev. 1924;4:215-250.

54. Winterberg H. Studien uber herzflimmern. 1. Uber die wirkung des N. Vagus und accelerans auf das flimmern des Herzens. Pflugers Arch Physiol. 1907;117:223-256.

55. Scherf D, Terranova R. Mechanism of auricular flutter and fibrillation. Am J Physiol. 1949;159:137-142.

56. Mayer AG. Rhythmical pulsation in Scyphomedusae. Carnegie Inst Wash. 1906;Paper 47:1-62.

57. Moe GK, Rheinboldt WC, Abildskov JA. A computer model of atrial fibrillation. Am Heart J. 1964;67:200-220.

58. Allessie M, Lammers W, Bonke F, et al. Experimental evaluation of Moe's multiple wavelet hypothesis of atrial fibrillation. In: Zipes, D., Jalife, J. (Eds.), Cardiac Electrophysiology and Arrhythmias. Grune and Stratton, New York. 1985:265-275.

59. Allessie MA, Bonke FI, Schopman FJ. Circus movement in rabbit atrial muscle as a mechanism of tachycardia. III. The "leading circle" concept: a new model of circus movement in cardiac tissue without the involvement of an anatomical obstacle. Circ Res. 1977;41(1):9-18.

60. Morillo CA, Klein GJ, Jones DL, et al. Chronic rapid atrial pacing. Structural, functional, and electrophysiological characteristics of a new model of sustained atrial fibrillation. Circulation. 1995;91(5):1588-1595.

61. Yue L, Feng J, Gaspo R, et al. Ionic remodeling underlying action potential changes in a canine model of atrial fibrillation. Circ Res. 1997;81(4):512-525. 
62. Ausma J, Dispersyn GD, Duimel H, et al. Changes in ultrastructural calcium distribution in goat atria during atrial fibrillation. J Mol Cell Cardiol. 2000;32(3):355-364.

63. Logan WF, Rowlands DJ, Howitt G, et al. Left atrial activity following cardioversion. Lancet. 1965;2(7410):471-473.

64. Manning WJ, Silverman DI, Katz SE, et al. Impaired left atrial mechanical function after cardioversion: relation to the duration of atrial fibrillation. J Am Coll Cardiol. 1994;23(7):1535-1540.

65. Schotten U, Duytschaever M, Ausma J, et al. Electrical and contractile remodeling during the first days of atrial fibrillation go hand in hand. Circulation. 2003;107(10):1433-1439.

66. Ausma J, Wijffels M, Thone F, et al. Structural changes of atrial myocardium due to sustained atrial fibrillation in the goat. Circulation. 1997;96(9):3157-3163.

67. Ausma J, van der Velden HM, Lenders MH, et al. Reverse structural and gap-junctional remodeling after prolonged atrial fibrillation in the goat. Circulation. 2003;107(15):2051-2058.

68. Thiedemann KU, Ferrans VJ. Left atrial ultrastructure in mitral valvular disease. Am J Pathol. 1977;89(3):575-604.

69. Allessie M, Ausma J, Schotten U. Electrical, contractile and structural remodeling during atrial fibrillation. Cardiovasc Res. 2002;54(2):230-246.

70. Spach MS, Boineau JP. Microfibrosis produces electrical load variations due to loss of side-toside cell connections: a major mechanism of structural heart disease arrhythmias. Pacing Clin Electrophysiol. 1997;20(2 Pt 2):397-413.

71. Van der Velden HM, van Kempen MJ, Wijffels MC, et al. Altered pattern of connexin40 distribution in persistent atrial fibrillation in the goat. J Cardiovasc Electrophysiol. 1998;9(6):596-607.

72. Takeuchi S, Akita T, Takagishi Y, et al. Disorganization of gap junction distribution in dilated atria of patients with chronic atrial fibrillation. Circ J. 2006;70(5):575-582.

73. De Groot N, Schalij M, Allessie M. Conduction properies of fibrillation waves in the epicardial plane in patients with acute and chronic atrial fibrillation. Pacing Clin Electrophysiol. 2006;29 Suppl 1:S1-S106.

74. Eckstein J, Verheule S, de Groot NM, et al. Mechanisms of perpetuation of atrial fibrillation in chronically dilated atria. Prog Biophys Mol Biol. 2008;97(2-3):435-451.

75. De Groot NM, Houben RP, Smeets JL, et al. Electropathological substrate of longstanding persistent atrial fibrillation in patients with structural heart disease: epicardial breakthrough. Circulation. 2010;122(17):1674-1682.

76. Jais $\mathrm{P}$, Haissaguerre $\mathrm{M}$, Shah DC, et al. A focal source of atrial fibrillation treated by discrete radiofrequency ablation. Circulation. 1997;95(3):572-576.

77. Schwartzman D, Bazaz R, Nosbisch J. Common left pulmonary vein: a consistent source of arrhythmogenic atrial ectopy. J Cardiovasc Electrophysiol. 2004;15(5):560-566.

78. Hsu LF, Jais P, Keane D, et al. Atrial fibrillation originating from persistent left superior vena cava. Circulation. 2004;109(7):828-832.

79. Hocini M, Ho SY, Kawara T, et al. Electrical conduction in canine pulmonary veins: electrophysiological and anatomic correlation. Circulation. 2002;105(20):2442-2448.

80. Perez-Lugones A, McMahon JT, Ratliff NB, et al. Evidence of specialized conduction cells in human pulmonary veins of patients with atrial fibrillation. J Cardiovasc Electrophysiol. 2003;14(8):803-809.

81. Atienza F, Almendral J, Moreno J, et al. Activation of inward rectifier potassium channels accelerates atrial fibrillation in humans: evidence for a reentrant mechanism. Circulation. 2006;114(23):2434-2442. 
82. Patterson E, Jackman WM, Beckman KJ, et al. Spontaneous pulmonary vein firing in man: relationship to tachycardia-pause early afterdepolarizations and triggered arrhythmia in canine pulmonary veins in vitro. J Cardiovasc Electrophysiol. 2007;18(10):1067-1075.

83. AFFIRM Investigators, Wyse DG, Waldo AL, et al. A comparison of rate control and rhythm control in patients with atrial fibrillation. N Engl J Med. 2002;347(23):1825-1833.

84. RACE Investigators, Van Gelder IC, Hagens VE, et al. A comparison of rate control and rhythm control in patients with recurrent persistent atrial fibrillation. N Engl J Med. 2002;347(23):18341840.

85. De Denus S, Sanoski CA, Carlsson J, et al. Rate vs rhythm control in patients with atrial fibrillation: a meta-analysis. Arch Intern Med. 2005;165(3):258-262.

86. Testa L, Biondi-Zoccai GG, Dello Russo A, et al. Rate-control vs. rhythm-control in patients with atrial fibrillation: a meta-analysis. Eur Heart J. 2005;26(19):2000-2006.

87. AF-CHF Investigators, Roy D, Talajic M, et al. Rhythm control versus rate control for atrial fibrillation and heart failure. N Engl J Med. 2008;358(25):2667-2677.

88. RACE II Investigators, Van Gelder IC, Groenveld HF, et al. Lenient versus strict rate control in patients with atrial fibrillation. $N$ Engl J Med. 2010;362(15):1363-1373.

89. Segal JB, McNamara RL, Miller MR, et al. The evidence regarding the drugs used for ventricular rate control. J Fam Pract. 2000;49(1):47-59.

90. Tamariz LJ, Bass EB. Pharmacological rate control of atrial fibrillation. Cardiol Clin. 2004;22(1):35-45.

91. Clemo HF, Wood MA, Gilligan DM, et al. Intravenous amiodarone for acute heart rate control in the critically ill patient with atrial tachyarrhythmias. Am J Cardiol. 1998;81(5):594-598.

92. Hunt SA, Abraham WT, Chin MH, et al. ACC/AHA 2005 Guideline Update for the Diagnosis and Management of Chronic Heart Failure in the Adult: a report of the American College of Cardiology/American Heart Association Task Force on Practice Guidelines. Circulation. 2005;112(12):e154-235.

93. Vaughan Williams EM. A classification of antiarrhythmic actions reassessed after a decade of new drugs. J Clin Pharmacol. 1984;24(4):129-147.

94. Dobrev D, Nattel S. New antiarrhythmic drugs for treatment of atrial fibrillation. Lancet. 2010;375(9721):1212-1223.

95. Mason PK, DiMarco JP. New pharmacological agents for arrhythmias. Circ Arrhythm Electrophysiol. 2009;2(5):588-597.

96. Smit MD, Van Gelder IC. Upstream therapy of atrial fibrillation. Expert Rev Cardiovasc Ther. 2009;7(7):763-778.

97. Hogue CW, Jr., Creswell LL, Gutterman DD, et al. Epidemiology, mechanisms, and risks: American College of Chest Physicians guidelines for the prevention and management of postoperative atrial fibrillation after cardiac surgery. Chest. 2005;128(2 Suppl):9S-16S.

98. Mathew JP, Parks R, Savino JS, et al. Atrial fibrillation following coronary artery bypass graft surgery: predictors, outcomes, and resource utilization. MultiCenter Study of Perioperative Ischemia Research Group. JAMA. 1996;276(4):300-306.

99. Aranki SF, Shaw DP, Adams DH, et al. Predictors of atrial fibrillation after coronary artery surgery. Current trends and impact on hospital resources. Circulation. 1996;94(3):390-397.

100. Kaireviciute D, Aidietis A, Lip GY. Atrial fibrillation following cardiac surgery: clinical features and preventative strategies. Eur Heart J. 2009;30(4):410-425.

101. Echahidi N, Pibarot P, O'Hara G, et al. Mechanisms, prevention, and treatment of atrial fibrillation after cardiac surgery. J Am Coll Cardiol. 2008;51(8):793-801. 
102. Davis EM, Packard KA, Hilleman DE. Pharmacologic prophylaxis of postoperative atrial fibrillation in patients undergoing cardiac surgery: beyond beta-blockers. Pharmacotherapy. 2010;30(7):749, 274e-318e.

103. McKeown PP, Gutterman D. Executive summary: American College of Chest Physicians guidelines for the prevention and management of postoperative atrial fibrillation after cardiac surgery. Chest. 2005;128(2 Suppl):1S-5S.

104. Dunning J, Treasure T, Versteegh $M$, et al. Guidelines on the prevention and management of de novo atrial fibrillation after cardiac and thoracic surgery. Eur J Cardiothorac Surg. 2006;30(6):852-872.

105. Sanjuan R, Blasco M, Carbonell N, et al. Preoperative use of sotalol versus atenolol for atrial fibrillation after cardiac surgery. Ann Thorac Surg. 2004;77(3):838-843.

106. Mitchell LB, Exner DV, Wyse DG, et al. Prophylactic oral amiodarone for the prevention of arrhythmias that begin early after revascularization, valve replacement, or repair: PAPABEAR: a randomized controlled trial. JAMA. 2005;294(24):3093-3100.

107. Cook RC, Humphries KH, Gin K, et al. Prophylactic intravenous magnesium sulphate in addition to oral beta-blockade does not prevent atrial arrhythmias after coronary artery or valvular heart surgery: a randomized, controlled trial. Circulation. 2009;120(11 Suppl):S163-169.

108. Fitzgerald JD. Class III antiarrhythmic agents: serendipity or drug design? Dialogues in Cardiovascular Medicine. 2004;9(4):243-252.

109. Singh BN. Amiodarone as paradigm for developing new drugs for atrial fibrillation. J Cardiovasc Pharmacol. 2008;52(4):300-305.

110. Charlier R, Delnaunois G, Bauthier J, et al. Recherches dans la série des benzofuranes. XL. Proprietétés antiarythmiques de l'amiodarone. Cardiologia. 1969;54:83-90.

111. Singh BN, Vaughan Williams EM. The effect of amiodarone, a new anti-anginal drug, on cardiac muscle. Br J Pharmacol. 1970;39(4):657-667.

112. Piccini JP, Berger JS, O'Connor CM. Amiodarone for the prevention of sudden cardiac death: a meta-analysis of randomized controlled trials. Eur Heart J. 2009;30(10):1245-1253.

113. Roy D, Talajic M, Dorian $P$, et al. Amiodarone to prevent recurrence of atrial fibrillation. Canadian Trial of Atrial Fibrillation Investigators. N Engl J Med. 2000;342(13):913-920.

114. AFFIRM Investigators. Maintenance of sinus rhythm in patients with atrial fibrillation: an AFFIRM substudy of the first antiarrhythmic drug. J Am Coll Cardiol. 2003;42(1):20-29.

115. Singh BN, Singh SN, Reda DJ, et al. Amiodarone versus sotalol for atrial fibrillation. $N$ Engl J Med. 2005;352(18):1861-1872.

116. Deedwania PC, Singh BN, Ellenbogen K, et al. Spontaneous conversion and maintenance of sinus rhythm by amiodarone in patients with heart failure and atrial fibrillation: observations from the veterans affairs congestive heart failure survival trial of antiarrhythmic therapy (CHF-STAT). Circulation. 1998;98(23):2574-2579.

117. Singh SN, Fletcher RD, Fisher SG, et al. Amiodarone in patients with congestive heart failure and asymptomatic ventricular arrhythmia. Survival Trial of Antiarrhythmic Therapy in Congestive Heart Failure. N Engl J Med. 1995;333(2):77-82.

118. Holt DW, Tucker GT, Jackson PR, et al. Amiodarone pharmacokinetics. Am Heart J. 1983;106(4 Pt 2):840-847.

119. Ha HR, Bigler $L$, Wendt $B$, et al. Identification and quantitation of novel metabolites of amiodarone in plasma of treated patients. Eur J Pharm Sci. 2005;24(4):271-279.

120. Trivier JM, Libersa $C$, Belloc $C$, et al. Amiodarone N-deethylation in human liver microsomes: involvement of cytochrome P450 3A enzymes. Life Sci. 1993;52(10):PL91-96. 
121. Adams PC, Holt DW, Storey GC, et al. Amiodarone and its desethyl metabolite: tissue distribution and morphologic changes during long-term therapy. Circulation. 1985;72(5):1064-1075.

122. Pollak PT, Bouillon T, Shafer SL. Population pharmacokinetics of long-term oral amiodarone therapy. Clin Pharmacol Ther. 2000;67(6):642-652.

123. Lafuente-Lafuente $C$, Alvarez JC, Leenhardt A, et al. Amiodarone concentrations in plasma and fat tissue during chronic treatment and related toxicity. Br J Clin Pharmacol. 2009;67(5):511519.

124. Kodama I, Kamiya K, Toyama J. Amiodarone: ionic and cellular mechanisms of action of the most promising class III agent. Am J Cardiol. 1999;84(9A):20R-28R.

125. Singh BN. Antiarrhythmic actions of amiodarone: a profile of a paradoxical agent. Am J Cardiol. 1996;78(4A):41-53.

126. Maruyama T, Richardson LC, Sun W, et al. Acute effects of amiodarone on membrane properties, refractoriness, and conduction in guinea pig papillary muscles. Heart Vessels. 1995;10(2):7886.

127. Kirchhof P, Degen H, Franz MR, et al. Amiodarone-induced postrepolarization refractoriness suppresses induction of ventricular fibrillation. J Pharmacol Exp Ther. 2003;305(1):257-263.

128. Burashnikov A, Di Diego JM, Sicouri S, et al. Atrial-selective effects of chronic amiodarone in the management of atrial fibrillation. Heart Rhythm. 2008;5(12):1735-1742.

129. Kosinski EJ, Albin JB, Young E, et al. Hemodynamic effects of intravenous amiodarone. J Am Coll Cardiol. 1984;4(3):565-570.

130. Halici Z, Dengiz GO, Odabasoglu F, et al. Amiodarone has anti-inflammatory and antioxidative properties: an experimental study in rats with carrageenan-induced paw edema. Eur J Pharmacol. 2007;566(1-3):215-221.

131. Traupe T, Keller M, Fojtu E, et al. Antioxidant activity and sex differences of acute vascular effects of amiodarone in advanced atherosclerosis. J Cardiovasc Pharmacol. 2007;50(5):578-584.

132. Breitenstein A, Stampfli SF, Camici GG, et al. Amiodarone inhibits arterial thrombus formation and tissue factor translation. Arterioscler Thromb Vasc Biol. 2008;28(12):2231-2238.

133. Courchesne WE. Characterization of a novel, broad-based fungicidal activity for the antiarrhythmic drug amiodarone. J Pharmacol Exp Ther. 2002;300(1):195-199.

134. Rosa SM, Antunes-Madeira MC, Matos MJ, et al. Lipid composition and dynamics of cell membranes of Bacillus stearothermophilus adapted to amiodarone. Biochim Biophys Acta. 2000;1487(2-3):286-295.

135. Benaim G, Sanders JM, Garcia-Marchan Y, et al. Amiodarone has intrinsic anti-Trypanosoma cruzi activity and acts synergistically with posaconazole. J Med Chem. 2006;49(3):892-899.

136. Stadler K, Ha HR, Ciminale V, et al. Amiodarone alters late endosomes and inhibits SARS coronavirus infection at a post endosomal level. Am J Respir Cell Mol Biol. 2008.

137. Theodossiou TA, Galanou MC, Paleos CM. Novel amiodarone-doxorubicin cocktail liposomes enhance doxorubicin retention and cytotoxicity in DU145 human prostate carcinoma cells. J Med Chem. 2008;51(19):6067-6074.

138. Mitterreiter S, Page RM, Kamp F, et al. Bepridil and amiodarone simultaneously target the Alzheimer's disease beta- and gamma-secretase via distinct mechanisms. J Neurosci. 2010;30(26):8974-8983.

139. Hohnloser SH, Klingenheben $\mathrm{T}$, Singh BN. Amiodarone-associated proarrhythmic effects. A review with special reference to torsade de pointes tachycardia. Ann Intern Med. 1994;121(7):529-535.

140. Zimetbaum P. Amiodarone for atrial fibrillation. N Engl J Med. 2007;356(9):935-941. 
141. Vassallo $P$, Trohman RG. Prescribing amiodarone: an evidence-based review of clinical indications. JAMA. 2007;298(11):1312-1322.

142. Van Erven L, Schalij MJ. Amiodarone: an effective antiarrhythmic drug with unusual side effects. Heart. 2010;96(19):1593-1600.

143. Spodick DH. Intrapericardial therapeutics and diagnostics. Am J Cardiol. 2000;85(8):1012-1014.

144. Mautz FR. Reduction of cardiac irritability by the epicardial and systemic administration of drugs as a protection in cardiac surgery. J Thorac Surg. 1936;5:612-628.

145. Seferovic PM, Ristic AD, Maksimovic R, et al. Initial clinical experience with PerDUCER device: promising new tool in the diagnosis and treatment of pericardial disease. Clin Cardiol. 1999;22(1 Suppl 1):I30-35.

146. Spodick DH. Physiology of the normal pericardium. In: The Pericardium. A Comprehensive textbook. Edited by Spodick DH. New York: Marcel Dekker. 1997:15-26.

147. Lerner-Tung MB, Chang AY, Ong LS, et al. Pharmacokinetics of intrapericardial administration of 5-fluorouracil. Cancer Chemother Pharmacol. 1997;40(4):318-320.

148. Stoll HP, Carlson K, Keefer LK, et al. Pharmacokinetics and consistency of pericardial delivery directed to coronary arteries: direct comparison with endoluminal delivery. Clin Cardiol. 1999;22(1 Suppl 1):I10-16.

149. Ujhelyi MR, Hadsall KZ, Euler DE, et al. Intrapericardial therapeutics: a pharmacodynamic and pharmacokinetic comparison between pericardial and intravenous procainamide delivery. J Cardiovasc Electrophysiol. 2002;13(6):605-611.

150. Boulanger B, Yuan Z, Flessner $M$, et al. Pericardial fluid absorption into lymphatic vessels in sheep. Microvasc Res. 1999;57(2):174-186.

151. Reddy PS, Curtiss EI, O'Toole JD, et al. Cardiac tamponade: hemodynamic observations in man. Circulation. 1978;58(2):265-272.

152. Dusting GJ, Nolan RD. Stimulation of prostacyclin release from the epicardium of anaesthetized dogs. Br J Pharmacol. 1981;74(3):553-562.

153. Miyazaki T, Zipes DP. Pericardial prostaglandin biosynthesis prevents the increased incidence of reperfusion-induced ventricular fibrillation produced by efferent sympathetic stimulation in dogs. Circulation. 1990;82(3):1008-1019.

154. Horkay F, Laine M, Szokodi I, et al. Human pericardial fluid contains the highest amount of endothelin-1 of all mammalian biologic fluids thus far tested. J Cardiovasc Pharmacol. 1995;26 Suppl 3:S502-504.

155. Kiss O, Geller L, Merkely B, et al. Endothelin-A-receptor antagonist LU 135.252 inhibits the formation of ventricular arrhythmias caused by intrapericardial infusion of endothelin-1. J Cardiovasc Pharmacol. 2000;36(5 Suppl 1):S317-319.

156. Kekesi V, Toma I, Nagy A, et al. Cardiovascular responses to catecholamines and interactions with endothelin-1 and adenine nucleosides in the pericardium of the dog heart. J Cardiovasc Pharmacol. 2004;44:S234-S238.

157. Nagy A, Toma I, Rusvai M, et al. Intrapericardial Adenine Nucleosides Induce Elevation of Endothelin-1 Concentration in the Pericardial Space of the Dog Heart. J Cardiovasc Pharmacol. 2004;44:S227-S230.

158. Nagy A, Sax B, Entz L, Jr., et al. Comparison of elimination and cardiovascular effects of adenine nucleosides administered intrapericardially or intravenously in anesthetized dog. J Cardiovasc Pharmacol. 2009;54(4):341-347.

159. Fujita M, Komeda M, Hasegawa K, et al. Pericardial fluid as a new material for clinical heart research. Int J Cardiol. 2001;77(2-3):113-118. 
160. Uchida Y, Yanagisawa-Miwa A, Nakamura F, et al. Angiogenic therapy of acute myocardial infarction by intrapericardial injection of basic fibroblast growth factor and heparin sulfate: an experimental study. Am Heart J. 1995;130(6):1182-1188.

161. Landau $C$, Jacobs AK, Haudenschild CC. Intrapericardial basic fibroblast growth factor induces myocardial angiogenesis in a rabbit model of chronic ischemia. Am Heart J. 1995;129(5):924-931.

162. Lazarous DF, Shou M, Stiber JA, et al. Pharmacodynamics of basic fibroblast growth factor: route of administration determines myocardial and systemic distribution. Cardiovasc Res. 1997;36(1):78-85.

163. Laham RJ, Rezaee M, Post M, et al. Intrapericardial delivery of fibroblast growth factor-2 induces neovascularization in a porcine model of chronic myocardial ischemia. J Pharmacol Exp Ther. 2000;292(2):795-802.

164. Laham RJ, Rezaee M, Post M, et al. Intrapericardial administration of basic fibroblast growth factor: myocardial and tissue distribution and comparison with intracoronary and intravenous administration. Catheter Cardiovasc Interv. 2003;58(3):375-381.

165. Maisch B, Ristic AD, Rupp H, et al. Pericardial access using the PerDUCER and flexible percutaneous pericardioscopy. Am J Cardiol. 2001;88(11):1323-1326.

166. Rodrigues AC, d'Avila A, Houghtaling $C$, et al. Intrapericardial echocardiography: a novel catheter-based approach to cardiac imaging. J Am Soc Echocardiogr. 2004;17(3):269-274.

167. Sosa E, Scanavacca M, d'Avila A, et al. A new technique to perform epicardial mapping in the electrophysiology laboratory. J Cardiovasc Electrophysiol. 1996;7(6):531-536.

168. Sosa E, Scanavacca M, d'Avila A, et al. Nonsurgical transthoracic epicardial catheter ablation to treat recurrent ventricular tachycardia occurring late after myocardial infarction. J Am Coll Cardiol. 2000;35(6):1442-1449.

169. Riviere CN, Patronik NA, Zenati MA. Prototype epicardial crawling device for intrapericardial intervention on the beating heart. Heart Surg Forum. 2004;7(6):E639-643.

170. Patronik NA, Zenati MA, Riviere CN. Preliminary evaluation of a mobile robotic device for navigation and intervention on the beating heart. Comput Aided Surg. 2005;10(4):225-232.

171. Roberts-Thomson KC, Seiler J, Steven D, et al. Percutaneous access of the epicardial space for mapping ventricular and supraventricular arrhythmias in patients with and without prior cardiac surgery. J Cardiovasc Electrophysiol. 2009.

172. Macris MP, Igo SR. Minimally invasive access of the normal pericardium: initial clinical experience with a novel device. Clin Cardiol. 1999;22(1 Suppl 1):I36-39.

173. Maisch B, Ristic AD, Seferovic PM, et al. Intrapericardial treatment of autoreactive myocarditis with triamcinolon. Successful administration in patients with minimal pericardial effusion. Herz. 2000;25(8):781-786.

174. Tio RA, Grandjean JG, Suurmeijer AJ, et al. Thoracoscopic monitoring for pericardial application of local drug or gene therapy. Int J Cardiol. 2002;82(2):117-121.

175. Rupp H, Rupp TP, Alter P, et al. Intrapericardial procedures for cardiac regeneration by stem cells: need for minimal invasive access (AttachLifter) to the normal pericardial cavity. Herz. 2010;35(7):458-465.

176. Verrier RL, Waxman S, Lovett EG, et al. Transatrial access to the normal pericardial space: a novel approach for diagnostic sampling, pericardiocentesis, and therapeutic interventions. Circulation. 1998;98(21):2331-2333.

177. Waxman S, Pulerwitz TC, Rowe KA, et al. Preclinical safety testing of percutaneous transatrial access to the normal pericardial space for local cardiac drug delivery and diagnostic sampling. Catheter Cardiovasc Interv. 2000;49(4):472-477. 
178. March KL, Woody M, Mehdi K, et al. Efficient in vivo catheter-based pericardial gene transfer mediated by adenoviral vectors. Clin Cardiol. 1999;22(1 Suppl 1):I23-29.

179. Kolettis TM, Kazakos N, Katsouras CS, et al. Intrapericardial drug delivery: pharmacologic properties and long-term safety in swine. Int J Cardiol. 2005;99(3):415-421.

180. Bartoli CR, Akiyama I, Godleski JJ, et al. Long-term pericardial catheterization is associated with minimum foreign-body response. Catheter Cardiovasc Interv. 2007;70(2):221-227.

181. Gleason JD, Nguyen KP, Kissinger KV, et al. Myocardial drug distribution pattern following intrapericardial delivery: an MRI analysis. J Cardiovasc Magn Reson. 2002;4(3):311-316.

182. Siden R, Kadish A, Flowers W, et al. Epicardial controlled-release verapamil prevents ventricular tachycardia episodes induced by acute ischemia in a canine model. J Cardiovasc Pharmacol. 1992;19(5):798-809.

183. Avitall B, Hare J, Zander G, et al. Iontophoretic transmyocardial drug delivery. A novel approach to antiarrhythmic drug therapy. Circulation. 1992;85(4):1582-1593.

184. Darsinos JT, Samouilidou EC, Krumholz B, et al. Distribution of lidocaine and digoxin in heart tissues and aorta following intrapericardial administration. Int J Clin Pharmacol Ther Toxicol. 1993;31(12):611-615.

185. Labhasetwar V, Kadish A, Underwood T, et al. The efficacy of controlled release D-sotalolpolyurethane epicardial implants for ventricular arrhythmias due to acute ischemia in dogs. Journal of Controlled Release. 1993;23(1):75-85.

186. Ayers GM, Rho TH, Ben-David J, et al. Amiodarone instilled into the canine pericardial sac migrates transmurally to produce electrophysiologic effects and suppress atrial fibrillation. $J$ Cardiovasc Electrophysiol. 1996;7(8):713-721.

187. Darsinos JT, Karli JN, Samouilidou EC, et al. Distribution of amiodarone in heart tissues following intrapericardial administration. Int J Clin Pharmacol Ther. 1999;37(6):301-306.

188. Van Brakel TJ, Hermans JJ, Accord RE, et al. Effects of intrapericardial sotalol and flecainide on transmural atrial electrophysiology and atrial fibrillation. J Cardiovasc Electrophysiol. 2009;20(2):207-215.

189. Hermans JJ, van Essen H, Struijker-Boudier HA, et al. Pharmacokinetic advantage of intrapericardially applied substances in the rat. J Pharmacol Exp Ther. 2002;301(2):672-678.

190. Maisch B, Seferovic PM, Ristic AD, et al. Guidelines on the diagnosis and management of pericardial diseases executive summary; The Task force on the diagnosis and management of pericardial diseases of the European society of cardiology. Eur Heart J. 2004;25(7):587-610.

191. Buselmeier TJ, Davin TD, Simmons RL, et al. Treatment of intractable uremic pericardial effusion. Avoidance of pericardiectomy with local steroid instillation. JAMA. 1978;240(13):13581359.

192. Maisch B, Ristic AD, Pankuweit S. Intrapericardial treatment of autoreactive pericardial effusion with triamcinolone; the way to avoid side effects of systemic corticosteroid therapy. Eur Heart J. 2002;23(19):1503-1508.

193. Winkler WB, Karnik R, Slany J. Treatment of exudative fibrinous pericarditis with intrapericardial urokinase. Lancet. 1994;344(8936):1541-1542.

194. Defouilloy C, Meyer G, Slama M, et al. Intrapericardial fibrinolysis: a useful treatment in the management of purulent pericarditis. Intensive Care Med. 1997;23(1):117-118.

195. Cui HB, Chen XY, Cui CC, et al. Prevention of pericardial constriction by transcatheter intrapericardial fibrinolysis with urokinase. Chin Med Sci J. 2005;20(1):5-10.

196. Ebner E, Runkewitz W, Fleck WF, et al. Transport of antibiotics to the endocardium following administration into the pericardial cavity-experimental study. Z Exp Chir Transplant Kunstliche Organe. 1985;18(6):353-356. 
197. Maisch B, Ristic AD, Pankuweit S, et al. Neoplastic pericardial effusion. Efficacy and safety of intrapericardial treatment with cisplatin. Eur Heart J. 2002;23(20):1625-1631.

198. Tomkowski WZ, Wisniewska J, Szturmowicz M, et al. Evaluation of intrapericardial cisplatin administration in cases with recurrent malignant pericardial effusion and cardiac tamponade. Support Care Cancer. 2004;12(1):53-57.

199. Bischiniotis TS, Antoniadou S, Katseas G, et al. Malignant cardiac tamponade in women with breast cancer treated by pericardiocentesis and intrapericardial administration of triethylenethiophosphoramide (thiotepa). Am J Cardiol. 2000;86(3):362-364.

200. Martinoni A, Cipolla CM, Cardinale D, et al. Long-term results of intrapericardial chemotherapeutic treatment of malignant pericardial effusions with thiotepa. Chest. 2004;126(5):1412-1416.

201. Liu G, Crump M, Goss PE, et al. Prospective comparison of the sclerosing agents doxycycline and bleomycin for the primary management of malignant pericardial effusion and cardiac tamponade. J Clin Oncol. 1996;14(12):3141-3147.

202. Kunitoh $H$, Tamura $T$, Shibata $T$, et al. A randomised trial of intrapericardial bleomycin for malignant pericardial effusion with lung cancer (JCOG9811). Br J Cancer. 2009;100(3):464-469.

203. Davis $S$, Rambotti $P$, Grignani F. Intrapericardial tetracycline sclerosis in the treatment of malignant pericardial effusion: an analysis of thirty-three cases. J Clin Oncol. 1984;2(6):631-636.

204. Shepherd FA, Morgan C, Evans WK, et al. Medical management of malignant pericardial effusion by tetracycline sclerosis. Am J Cardiol. 1987;60(14):1161-1166.

205. Maher EA, Shepherd FA, Todd TJ. Pericardial sclerosis as the primary management of malignant pericardial effusion and cardiac tamponade. J Thorac Cardiovasc Surg. 1996;112(3):637-643.

206. Arndt JO, Pasch U, Samodelov LF, et al. Reversible blockade of myelinated and nonmyelinated cardiac afferents in cats by instillation of procaine into the pericardium. Cardiovasc Res. 1981;15(2):61-67.

207. Samodelov LF, Pohl M, Arndt JO. Reversible blockade of cardiac efferents with procaine instilled into the pericardium of cats. Cardiovasc Res. 1982;16(4):187-193.

208. Dorward PK, Flaim M, Ludbrook J. Blockade of cardiac nerves by intrapericardial local anaesthetics in the conscious rabbit. Aust J Exp Biol Med Sci. 1983;61 (Pt 2):219-230.

209. O'Donnell CP, Scheuer DA, Keil LC, et al. Cardiac nerve blockade by infusion of procaine into the pericardial space of conscious dogs. Am J Physiol. 1991;260(6 Pt 2):R1176-1182.

210. Evans RG, Hayes IP, Ludbrook J, et al. Factors confounding blockade of cardiac afferents by intrapericardial procaine in conscious rabbits. Am J Physiol. 1993;264(6 Pt 2):H1861-1870.

211. Lew MJ, Ludbrook J, Pavia JM, et al. Selective manipulation of neurohumoral control of the cardiac pacemaker by drugs given intrapericardially. J Pharmacol Methods. 1987;17(2):137-148.

212. Moreno $R$, Waxman $S$, Rowe $K$, et al. Intrapericardial beta-adrenergic blockade with esmolol exerts a potent antitachycardic effect without depressing contractility. J Cardiovasc Pharmacol. 2000;36(6):722-727.

213. Van Brakel TJ, Hermans JJ, Janssen BJ, et al. Intrapericardial delivery enhances cardiac effects of sotalol and atenolol. J Cardiovasc Pharmacol. 2004;44(1):50-56.

214. Vereckei A, Gorski JC, Ujhelyi M, et al. Intrapericardial ibutilide administration fails to terminate pacing-induced sustained atrial fibrillation in dogs. Cardiovasc Drugs Ther. 2004;18(4):269277.

215. Carvas M, Nascimento BC, Acar M, et al. Intrapericardial ranolazine prolongs atrial refractory period and markedly reduces atrial fibrillation inducibility in the intact porcine heart. J Cardiovasc Pharmacol. 2010;55(3):286-291. 
216. Willerson JT, Igo SR, Yao SK, et al. Localized administration of sodium nitroprusside enhances its protection against platelet aggregation in stenosed and injured coronary arteries. Tex Heart Inst J. 1996;23(1):1-8.

217. Fei L, Baron AD, Henry DP, et al. Intrapericardial delivery of L-arginine reduces the increased severity of ventricular arrhythmias during sympathetic stimulation in dogs with acute coronary occlusion: nitric oxide modulates sympathetic effects on ventricular electrophysiological properties. Circulation. 1997;96(11):4044-4049.

218. Waxman S, Moreno R, Rowe KA, et al. Persistent primary coronary dilation induced by transatrial delivery of nitroglycerin into the pericardial space: a novel approach for local cardiac drug delivery. J Am Coll Cardiol. 1999;33(7):2073-2077.

219. Kumar K, Nguyen K, Waxman $S$, et al. Potent antifibrillatory effects of intrapericardial nitroglycerin in the ischemic porcine heart. J Am Coll Cardiol. 2003;41(10):1831-1837.

220. Kikuchi A, Okano T. Pulsatile drug release control using hydrogels. Adv Drug Deliv Rev. 2002;54(1):53-77.

221. Kellar RS, Landeen LK, Shepherd BR, et al. Scaffold-based three-dimensional human fibroblast culture provides a structural matrix that supports angiogenesis in infarcted heart tissue. Circulation. 2001;104(17):2063-2068.

222. Kellar RS, Shepherd BR, Larson DF, et al. Cardiac patch constructed from human fibroblasts attenuates reduction in cardiac function after acute infarct. Tissue Eng. 2005;11(11/12):16781687.

223. Kutschka I, Chen IY, Kofidis T, et al. Collagen matrices enhance survival of transplanted cardiomyoblasts and contribute to functional improvement of ischemic rat hearts. Circulation. 2006;114(1 Suppl):I167-173.

224. Konertz WF, Kostelka M, Mohr FW, et al. Reducing the incidence and severity of pericardial adhesions with a sprayable polymeric matrix. Ann Thorac Surg. 2003;76(4):1270-1274.

225. Putnam D. Drug delivery: the heart of the matter. Nat Mater. 2008;7(11):836-837.

226. Davis KA, Anseth KS. Controlled release from crosslinked degradable networks. Crit Rev Ther Drug Carrier Syst. 2002;19(4-5):385-423.

227. Tomaske M, Gerritse B, Kretzers L, et al. A 12-year experience of bipolar steroid-eluting epicardial pacing leads in children. Ann Thorac Surg. 2008;85(5):1704-1711.

228. Mond HG, Stokes KB. The steroid-eluting electrode: a 10-year experience. Pacing Clin Electrophysiol. 1996;19(7):1016-1020.

229. Chorny M, Mishaly D, Leibowitz A, et al. Site-specific delivery of dexamethasone from biodegradable implants reduces formation of pericardial adhesions in rabbits. J Biomed Mater Res A. 2006.

230. Yoo D, Vinten-Johansen J, Schmarkey LS, et al. Adhesive epicardial corticosteroids prevent postoperative atrial fibrillation. Circ Arrhythm Electrophysiol. 2010;3(5):505-510.

231. Sintov A, Scott WA, Gallagher KP, et al. Conversion of ouabain-induced ventricular tachycardia in dogs with epicardial lidocaine: pharmacodynamics and functional effects. Pharm Res. 1990;7(1):28-33.

232. Sintov A, Scott WA, Siden R, et al. Efficacy of epicardial controlled-release lidocaine for ventricular tachycardia induced by rapid ventricular pacing in dogs. J Cardiovasc Pharmacol. 1990;16(5):812-817.

233. Siden R, Flowers WE, Levy RJ. Epicardial propranolol administration for ventricular arrhythmias in dogs: matrix formulation and characterization. Biomaterials. 1992;13(11):764-770. 
234. Labhasetwar V, Underwood T, Gallagher M, et al. Sotalol controlled-release systems for arrhythmias: in vitro characterization, in vivo drug disposition, and electrophysiologic effects. J Pharm Sci. 1994;83(2):156-164.

235. Labhasetwar V, Underwood $\mathrm{T}$, Schwendeman SP, et al. Iontophoresis for modulation of cardiac drug delivery in dogs. Proc Natl Acad Sci U S A. 1995;92(7):2612-2616.

236. Kikuchi K, McDonald AD, Sasano T, et al. Targeted modification of atrial electrophysiology by homogeneous transmural atrial gene transfer. Circulation. 2005;111(3):264-270.

237. Amit G, Kikuchi K, Greener ID, et al. Selective molecular potassium channel blockade prevents atrial fibrillation. Circulation. 2010;121(21):2263-2270.

238. Mei L, Jin X, Song C, et al. Immobilization of gene vectors on polyurethane surfaces using a monoclonal antibody for localized gene delivery. J Gene Med. 2006;8(6):690-698.

239. Hohnloser SH, Crijns HJ, van Eickels M, et al. Effect of dronedarone on cardiovascular events in atrial fibrillation. N Engl J Med. 2009;360(7):668-678. 


\section{CHAPTER 2}

Determination of amiodarone and

dronedarone, and their principal

metabolites in plasma and myocardium by

high-performance liquid chromatography

and UV-detection

Published as:

Robert W. Bolderman, J.J. Rob Hermans, Jos G. Maessen. Determination of the class III antiarrhythmic drugs dronedarone and amiodarone, and their principal metabolites in plasma and myocardium by high-performance liquid chromatography and UV-detection.

J Chromatogr B Analyt Technol Biomed Life Sci. 2009;877(18-19):1727-31. 


\section{ABSTRACT}

Amiodarone is one of the most effective and widely used antiarrhythmic drugs. Dronedarone, a noniodinated benzofuran derivative of amiodarone, is believed to have a better side effect profile, and is currently undergoing phase III clinical trials. A novel method was developed for the determination of amiodarone, dronedarone, and their principal metabolites desethylamiodarone and debutyldronedarone in both plasma and myocardial tissue by high-performance liquid chromatography (HPLC) coupled with UV-detection. Samples were obtained from healthy humans (plasma) and goats (plasma and myocardium). Sample preparation included deproteinization with acetonitrile and extraction with a mixture of heptane and dichloromethane (50/50, v/v). Chromatographic separation was performed on a Pathfinder PS polymeric C18 column (50 x $4.6 \mathrm{~mm}, 2.5 \mu \mathrm{m})$ with a mobile phase of acetonitrile, isopropanol, water and ammonia (80/10/10/0.025, v/v/v/v) at a flow-rate of 1 $\mathrm{ml} / \mathrm{min}$. Calibration curves of all analytes were linear in the range $0.01-5 \mu \mathrm{g} / \mathrm{ml}$ for plasma samples, with a lower limit of quantification (LLOQ) of $0.04 \mu \mathrm{g} / \mathrm{ml}$. For myocardial tissue samples, linear curves of all analytes were observed in the range of $0.02-500 \mu \mathrm{g} / \mathrm{g}$, with a LLOQ of $0.08 \mu \mathrm{g} / \mathrm{g}$. Within- and between-day precision was $<18 \%$, and within- and between-day accuracy ranged from 97.5 to $109.7 \%$, with a recovery of $67.6-79.9 \%$. The present method enables sensitive and specific detection of amiodarone, dronedarone and principal metabolites in plasma as well as myocardial tissue. 


\section{INTRODUCTION}

Amiodarone, a benzofuran derivative synthesized in 1962 as a coronary vasodilator, is now recognized as one of the most effective drugs in the treatment of both atrial and ventricular arrhythmias. ${ }^{1}$ It exerts very complex electropharmacologic effects, including class I, II, III and IV antiarrhythmic actions, with marked differences between short-term and long-term effects. ${ }^{2}$ Despite its potent efficacy, long-term use of amiodarone is limited by serious side effects such as thyroid dysfunction, pulmonary toxicity and hepatic toxicity. ${ }^{3}$ Unusual pharmacokinetic characteristics, especially the large volume of distribution and long elimination half-life (up to 6 months) of both the parent drug and its primary metabolite desethylamiodarone, further complicate the clinical use of amiodarone.

Since most of the unfavourable properties of amiodarone are probably due to the presence of its iodine moiety, several non-iodinated benzofuran derivatives have been synthesized. Of these modified analogues, dronedarone is most advanced in clinical development. ${ }^{1}$ Like amiodarone, dronedarone blocks multiple ion currents and shares the low potential for causing torsades de pointes. Dronedarone has a serum half-life of about 24 hours and does not significantly accumulate in plasma or tissue. This also holds true for its principal metabolite debutyldronedarone, which possesses less pharmacologic activity. Recent phase III trials comparing dronedarone treatment with placebo in patients with atrial fibrillation showed efficacy of the drug, while adverse events did not significantly differ between both groups. 4,5 Therefore, dronedarone is expected to receive regulatory approval for several indications concerning atrial fibrillation.

Many assays for the determination of amiodarone and desethylamiodarone in plasma using high-performance liquid chromatography with UV detection (HPLCUV) have been described in the literature, of which 40 procedures were compared in a review by Pollak. ${ }^{6}$ In recent years, HPLC has also been coupled to mass spectrometry and chemiluminescent detection for even more specific and sensitive quantification of amiodarone and desethylamiodarone..$^{7-11}$ To the best of our knowledge, no detailed high-performance liquid chromatography assay for the determination of dronedarone and debutyldronedarone has been described so far. Therefore, the primary aim of the present study was to develop an HPLC method for the determination of dronedarone and debutyldronedarone in plasma as well as in the therapeutic target, i.e. in myocardial tissue. Furthermore this assay was validated for determination of amiodarone and desethylamiodarone. Thus we here present a uniform assay that can be applied for detection of both dronedarone and amiodarone as well as their principal metabolites. 


\section{EXPERIMENTAL}

\section{Chemicals}

Dronedarone, debutyldronedarone, amiodarone and desethylamiodarone were a gift from Sanofi-Aventis (Sisteron, France) (Figure 1). 4,4'-Methylenebis(2,6diisopropyl-N,N-dimethylaniline) (internal standard) and glycine were purchased from Sigma-Aldrich (St. Louis, MO, USA). Trifluoroacetic acid, ammonia (25\%) and sodium hydroxide were obtained from Merck (Darmstadt, Germany). HPLC grade methanol, heptane, dichloromethane, acetonitrile and isopropanolol were supplied by Biosolve (Valkenswaard, The Netherlands). A Milli-Q Plus water purification system (Millipore, Amsterdam, The Netherlands) was used to obtain purified water. All other reagents were of analytical grade.

\section{Dronedarone}

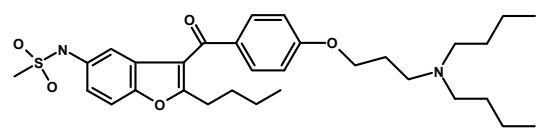

Debutyldronedarone

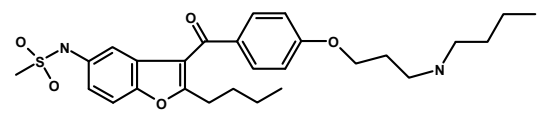

Amiodarone<smiles>CCCCc1oc2ccccc2c1C(=O)c1cc(C)c(OCCN(CC)CC)c(C)c1</smiles>

Desethylamiodarone<smiles>CCCCc1oc2ccccc2c1C(=O)c1cc(C)c(OCCNCC)c(I)c1</smiles>

Internal standard<smiles>CC(C)c1cc(Cc2cc(C(C)C)c(C(C)C)c(C(C)C)c2)cc(C(C)C)c1C(C)C</smiles>

Figure 1. Chemical structures of dronedarone, debutyldronedarone, amiodarone, desethylamiodarone, and internal standard.

\section{Biological Samples}

Samples were obtained from healthy volunteers (plasma) and goats (plasma and left ventricular myocardial tissue). Blood was drawn from a peripheral vein and collected in EDTA-containing vacutainer tubes (Becton-Dickinson, Franklin Lakes, 
New Jersey, USA) and centrifuged (at $3500 \times \mathrm{g}, 10 \mathrm{~min}, 4{ }^{\circ} \mathrm{C}$ ) to obtain plasma. Left ventricular tissue samples were homogenized in water $(1: 3, \mathrm{w} / \mathrm{v})$ with a tissue blender. Plasma and tissue homogenates were stored at $-80{ }^{\circ} \mathrm{C}$.

\section{HPLC-UV Analysis}

The HPLC system consisted of a Shimadzu LC-10AD liquid chromatograph, a SIL10AD autoinjector and a SP8490 variable wavelength UV-detector (Newport Spectra-Physics, Utrecht, The Netherlands) set at $254 \mathrm{~nm}$. LabSolutions software (Shimadzu, Kyoto, Japan) was used for instrument control and data acquisition. HPLC separations were performed on a Pathfinder PS polymeric C18 column (50 x 4.6 $\mathrm{mm}, 2.5 \mu \mathrm{m}$ ) (Shimadzu, Kyoto, Japan) as stationary phase. Each 10-minute chromatographic run was carried out at a flow-rate of $1 \mathrm{ml} / \mathrm{min}$ with a mobile phase of acetonitrile, isopropanol, water and ammonia (80/10/10/0.025, v/v/v/v).

\section{Standard Solutions}

Stock solutions of $2000 \mu \mathrm{g} / \mathrm{ml}$ of dronedarone, debutyldronedarone, amiodarone, desethylamiodarone and internal standard were separately prepared in methanol and diluted and combined to obtain working solutions. Stock solutions were stored at $-20{ }^{\circ} \mathrm{C}$ and working solutions were kept refrigerated $\left(2-6{ }^{\circ} \mathrm{C}\right)$ and protected from light. The working solutions were used to prepare twelve calibrators in blank plasma $(0.01,0.02,0.04,0.08,0.16,0.32,0.64,1.0,2.0,3.0,4.0$ and $5.0 \mu \mathrm{g} / \mathrm{ml})$ and blank tissue homogenates $(0.005,0.01,0.02,0.04,0.08,0.25,1.25,6.25,12.5,25,62.5$ and $125 \mu \mathrm{g} / \mathrm{ml}$, corresponding to wet tissue concentrations of $0.02,0.04,0.08,0.16$, $0.32,1,5,25,50,100,250$ and $500 \mu \mathrm{g} / \mathrm{g}$, respectively). Quality control samples were prepared in a similar way with plasma concentrations of $0.04,1$ and $5 \mu \mathrm{g} / \mathrm{ml}$, and tissue homogenate concentrations of $0.02,0.25$ and $25 \mu \mathrm{g} / \mathrm{ml}$, corresponding to wet tissue concentrations of $0.08,1$ and $100 \mu \mathrm{g} / \mathrm{g}$, respectively.

\section{Extraction Procedure}

To a $10-\mathrm{ml}$ glass centrifuge tube, $400 \mu \mathrm{l}$ of plasma or tissue homogenate, $100 \mu \mathrm{l}$ of working solution (containing $100 \mu \mathrm{g} / \mathrm{ml}$ internal standard), $100 \mu \mathrm{l}$ of $1 \mathrm{M}$ glycine buffer ( $\mathrm{pH}$ 1.0) and $5 \mathrm{ml}$ of acetonitrile were added. After precipitation of the protein fraction (at $5000 \times \mathrm{g}, 10 \mathrm{~min}, 4{ }^{\circ} \mathrm{C}$ ), the supernatant was transferred to a second $10-\mathrm{ml}$ glass centrifuge tube and evaporated to dryness under nitrogen at $37{ }^{\circ} \mathrm{C}$. To the residue, $1 \mathrm{ml}$ of $1 \mathrm{M}$ glycine buffer $(\mathrm{pH} 10.5)$ and $2 \mathrm{ml}$ of a mixture of heptane and dichloromethane (50/50, v/v) were added. After extraction and centrifugation (at $5000 \times \mathrm{g}, 10 \mathrm{~min}, 4{ }^{\circ} \mathrm{C}$ ), the supernatant was transferred to a third 10 - $\mathrm{ml}$ glass 
centrifuge tube and evaporated to dryness under nitrogen at $37^{\circ} \mathrm{C}$. The residue was reconstituted with $50 \mu \mathrm{l}$ of mobile phase and was transferred to an autoinjector vial. A $10-\mu 1$ aliquot of the reconstituted extract was injected by the autoinjector.

\section{Method Validation}

Linearity of the assay was assessed using twelve-point calibration curves (prepared in triplicate). Peak area ratios of dronedarone, debutyldronedarone, amiodarone and desethylamiodarone to internal standard were plotted against the corresponding concentrations and linear regression was performed using a $1 / x$ weighted linear regression method. Within-day and between-day accuracy and precision were assessed in quality control samples at three concentrations (with plasma concentrations of $0.04,1$ and $5 \mu \mathrm{g} / \mathrm{ml}$, and tissue homogenate concentrations of $0.02,0.25$ and $25 \mu \mathrm{g} / \mathrm{ml}$, corresponding to wet tissue concentrations of $0.08,1$ and $100 \mu \mathrm{g} / \mathrm{g}$, respectively). For within-day measurements, five independently prepared samples for each concentration level were injected on the same day with two-hour intervals. For between-day measurements, sample preparations and injections were performed on five different days. Accuracy was expressed as relative error (\%RE) between the mean found concentration and the spiked concentration. Precision was expressed as relative standard deviation (\%RSD), being the ratio between the standard deviation and the mean found concentration. The limit of detection (LOD) was defined as the lowest concentration on the calibration curve with a signal-to-noise ratio of $\geq 3$. Lower limit of quantification (LLOQ) was defined as the lowest concentration on the calibration curve that can be measured with a RE of $< \pm 20 \%$ and a RSD of $<20 \%$. Recovery was calculated as the ratio of mean peak area from extracted quality control samples to the mean peak area from directly injected standards determined at three concentrations (with plasma concentrations of $0.04,1$ and $5 \mu \mathrm{g} / \mathrm{ml}$, and tissue homogenate concentrations of $0.02,0.25$ and $25 \mu \mathrm{g} / \mathrm{ml}$, corresponding to wet tissue concentrations of $0.08,1$ and $100 \mu \mathrm{g} / \mathrm{g}$, respectively) in triplicate. 


\section{RESUlts}

\section{Optimization of HPLC Conditions}

HPLC separations were initially tested on five types of Pathfinder polymeric C18 columns (AS, AP, EP, MR and PS). The PS column provided the best peak shapes combined with relatively short retention times and was used for futher analysis. Various mixtures were evaluated as mobile phase, differing in $\mathrm{pH}$ and in volume percentages of acetonitrile, isopropanol and water. A mobile phase with volume percentages of $80 \%$ acetonitrile, $10 \%$ isopropanol, $10 \%$ water and $0.025 \%$ ammonia (producing a $\mathrm{pH}$ of 10.5), yielded good peak shapes with retention times of 2.7, 3.1, 4.3 and 5.6 minutes for dronedarone, debutyldronedarone, desethylamiodarone and amiodarone, respectively. Three compounds were screened as internal standard. Promethazine and tamoxifen had retention times of less than 2 minutes and were liable to early interfering peaks. 4,4'-Methylenebis(2,6-diisopropyl-N,N-dimethylaniline) had a retention time of 7.5 minutes, 2 minutes longer than the retention time of amiodarone, and was examined as internal standard during further assay validation.

\section{Optimization of Extraction}

After deproteinization, interference was present in chromatograms of reconstituted acetonitrile residues. This could be minimized by addition of glycine buffer $(\mathrm{pH}$ 10.5) to acetonitrile residues and subsequent extraction with a mixture of heptane and dichloromethane. Although recovery decreased, the resulting chromatograms allowed for increased sensitivity and specificity. Representative chromatograms after extraction of plasma samples and tissue homogenates are shown in Figures 2 and 3. 
A

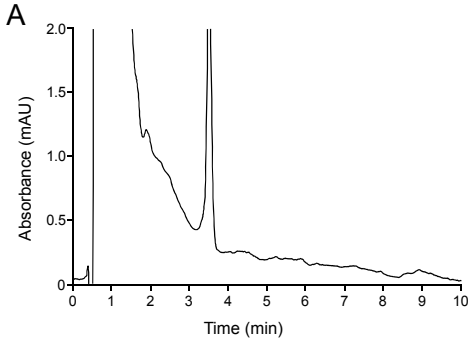

C

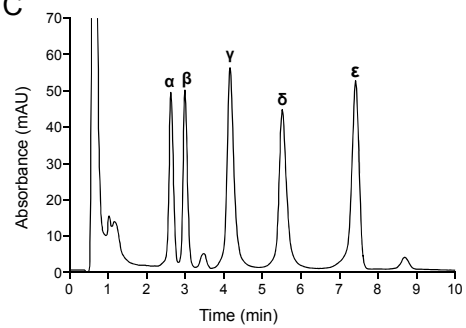

B

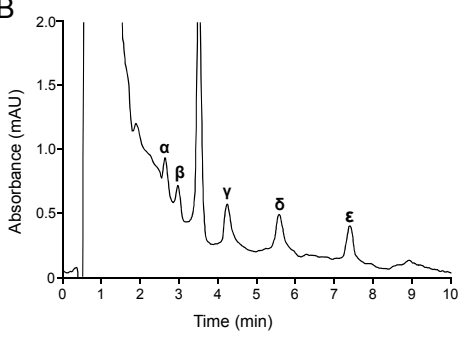

Figure 2. Representative chromatograms after extraction of (A) blank human plasma, (B) blank human plasma spiked with $0.04 \mu \mathrm{g} / \mathrm{ml}$ (lower limit of quantification) and (C) blank human plasma spiked with $5 \mu \mathrm{g} / \mathrm{ml}$ of $(\alpha)$ dronedarone, $(\beta)$ debutyldronedarone, $(\gamma)$ desethylamiodarone and $(\delta)$ amiodarone. Spiked concentrations of $(\varepsilon)$ internal standard were five times higher than analyte concentrations.
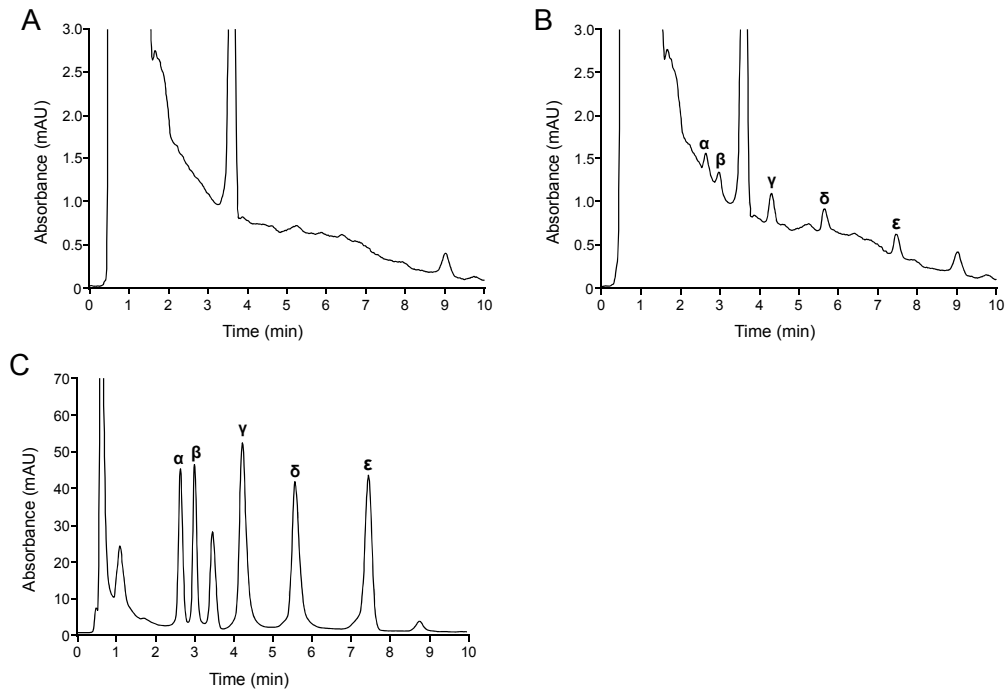

Figure 3. Representative chromatograms after extraction of (A) blank goat myocardial tissue homogenate, (B) blank goat myocardial tissue homogenate spiked for corresponding wet tissue concentrations of $0.08 \mu \mathrm{g} / \mathrm{g}$ (lower limit of quantification) and (C) blank goat myocardial tissue homogenate spiked for corresponding wet tissue concentrations of $20 \mu \mathrm{g} / \mathrm{g}$ of $(\alpha)$ dronedarone, $(\beta)$ debutyldronedarone, $(\gamma)$ desethylamiodarone and $(\delta)$ amiodarone. Spiked concentrations of $(\varepsilon)$ internal standard were five times higher than analyte concentrations. 
Table 1. Slope and intercept of calibration curves in spiked plasma (human and goat) and myocardial tissue (goat)

\begin{tabular}{lrrrr}
\hline & Dronedarone & Debutyldronedarone & Amiodarone & Desethylamiodarone \\
\hline Plasma (human) & & & & \\
$\quad$ Slope \pm SD & $0.0314 \pm 0.0007$ & $0.0329 \pm 0.0007$ & $0.0487 \pm 0.0009$ & $0.0541 \pm 0.0012$ \\
Intercept \pm SD & $-0.0019 \pm 0.0010$ & $-0.0009 \pm 0.0008$ & $-0.0010 \pm 0.0004$ & $-0.0025 \pm 0.0018$ \\
& & & & \\
Plasma (goat) & & & & \\
Slope \pm SD & $0.0312 \pm 0.0007$ & $0.0330 \pm 0.0008$ & $0.0486 \pm 0.0011$ & $0.0540 \pm 0.0014$ \\
Intercept \pm SD & $-0.0016 \pm 0.0008$ & $-0.0011 \pm 0.0007$ & $-0.0013 \pm 0.0006$ & $-0.0022 \pm 0.0019$ \\
Myocardium (goat) & & & & \\
$\quad$ & & & & \\
Slope \pm SD & $0.0321 \pm 0.0002$ & $0.0343 \pm 0.0001$ & $0.0506 \pm 0.0011$ & $0.0565 \pm 0.0010$ \\
Intercept \pm SD & $-0.0013 \pm 0.0008$ & $-0.0016 \pm 0.0003$ & $-0.0017 \pm 0.0016$ & $-0.0019 \pm 0.0007$ \\
\hline
\end{tabular}

\section{Method Validation}

Twelve-point calibration curves, prepared in triplicate, showed a good linearity (regression coefficients $>0.999$ ) for all analytes in human and goat plasma samples (range $0.01-5 \mu \mathrm{g} / \mathrm{ml}$ ) and tissue homogenates (corresponding wet tissue concentration range of $0.02-500 \mu \mathrm{g} / \mathrm{g}$ ) (Table 1). Within- and between-day precision (expressed as relative standard deviation) was $<18 \%$, within- and between-day accuracy (expressed as relative error) ranged from -2.5 to $9.7 \%$, and recovery ranged from 67.6 to $79.9 \%$ (Table 2). The limit of detection (LOD) and lower limit of quantification (LLOQ) for all analytes in plasma samples were 0.01 and $0.04 \mu \mathrm{g} / \mathrm{ml}$, respectively. For myocardial tissue samples, LOD and LLOQ were 0.02 and $0.08 \mu \mathrm{g} / \mathrm{g}$, respectively. 
Table 2. Precision, accuracy and recovery (C, concentration; RSD, relative standard deviation; RE, relative error; Rec, recovery)

\begin{tabular}{|c|c|c|c|c|c|c|c|c|}
\hline & \multirow[b]{2}{*}{ Spiked C } & \multicolumn{3}{|c|}{ Within-day } & \multicolumn{3}{|c|}{ Between-day } & \multirow[b]{2}{*}{$\operatorname{Rec}(\%)$} \\
\hline & & Found C & $\operatorname{RSD}(\%)$ & $\mathrm{RE}(\%)$ & Found C & $\mathrm{RSD}(\%)$ & $\mathrm{RE}(\%)$ & \\
\hline Plasma (human) & $(\mu \mathrm{g} / \mathrm{ml})$ & $(\mu \mathrm{g} / \mathrm{ml})$ & & & $(\mu \mathrm{g} / \mathrm{ml})$ & & & \\
\hline \multirow[t]{3}{*}{ Dronedarone } & 0.04 & 0.043 & 13.9 & 6.1 & 0.043 & 16.3 & 6.9 & 74.5 \\
\hline & 1 & 0.979 & 2.6 & -2.1 & 0.989 & 3.3 & -1.1 & 76.6 \\
\hline & 5 & 5.053 & 3.2 & 1.1 & 4.979 & 2.0 & -0.4 & 78.2 \\
\hline \multirow[t]{3}{*}{ Debutyldronedarone } & 0.04 & 0.043 & 12.8 & 7.1 & 0.043 & 17.2 & 8.1 & 75.6 \\
\hline & 1 & 0.986 & 2.6 & -1.4 & 0.993 & 3.5 & -0.7 & 76.9 \\
\hline & 5 & 5.065 & 2.8 & 1.3 & 5.015 & 1.2 & 0.3 & 79.0 \\
\hline \multirow[t]{3}{*}{ Amiodarone } & 0.04 & 0.043 & 16.4 & 7.0 & 0.044 & 15.7 & 9.4 & 67.8 \\
\hline & 1 & 1.011 & 4.3 & 1.1 & 1.015 & 4.0 & 1.5 & 70.7 \\
\hline & 5 & 5.107 & 3.3 & 2.1 & 4.979 & 1.3 & -0.4 & 71.8 \\
\hline \multirow{3}{*}{ Desethylamiodarone } & 0.04 & 0.043 & 15.6 & 6.5 & 0.043 & 16.7 & 8.0 & 72.0 \\
\hline & 1 & 0.986 & 3.6 & -1.4 & 1.008 & 3.3 & 0.8 & 74.7 \\
\hline & 5 & 4.964 & 2.4 & -0.7 & 5.105 & 2.7 & 2.1 & 73.7 \\
\hline \multicolumn{9}{|l|}{ Plasma (goat) } \\
\hline \multirow[t]{3}{*}{ Dronedarone } & 0.04 & 0.042 & 11.5 & 5.6 & 0.041 & 15.0 & 6.4 & 73.9 \\
\hline & 1 & 0.975 & 2.2 & -2.5 & 0.985 & 3.4 & -1.5 & 75.5 \\
\hline & 5 & 5.031 & 2.9 & 0.6 & 4.961 & 2.5 & -0.8 & 77.7 \\
\hline \multirow[t]{3}{*}{ Debutyldronedarone } & 0.04 & 0.043 & 13.1 & 6.4 & 0.043 & 17.6 & 7.0 & 74.6 \\
\hline & 1 & 0.981 & 2.4 & -1.9 & 0.989 & 3.6 & -1.1 & 76.1 \\
\hline & 5 & 5.043 & 2.5 & 0.9 & 4.945 & 1.7 & -1.1 & 78.3 \\
\hline \multirow[t]{3}{*}{ Amiodarone } & 0.04 & 0.043 & 15.7 & 6.4 & 0.044 & 14.7 & 8.8 & 67.6 \\
\hline & 1 & 1.007 & 4.7 & 0.7 & 1.026 & 3.7 & 2.6 & 70.3 \\
\hline & 5 & 5.086 & 3.6 & 1.7 & 4.958 & 1.2 & -0.8 & 71.5 \\
\hline \multirow[t]{3}{*}{ Desethylamiodarone } & 0.04 & 0.042 & 15.1 & 5.9 & 0.043 & 16.3 & 7.6 & 72.1 \\
\hline & 1 & 0.982 & 3.2 & -1.8 & 1.003 & 3.7 & 0.3 & 74.2 \\
\hline & 5 & 4.943 & 2.2 & -1.1 & 5.088 & 3.3 & 1.8 & 73.4 \\
\hline Myocardium (goat) & $(\mu \mathrm{g} / \mathrm{g})$ & $(\mu \mathrm{g} / \mathrm{g})$ & & & $(\mu \mathrm{g} / \mathrm{g})$ & & & \\
\hline \multirow[t]{3}{*}{ Dronedarone } & 0.08 & 0.087 & 9.7 & 9.0 & 0.088 & 12.0 & 9.7 & 69.9 \\
\hline & 1 & 0.992 & 3.7 & -0.8 & 1.007 & 4.6 & 0.7 & 74.7 \\
\hline & 100 & 101.687 & 2.1 & 1.7 & 101.033 & 3.1 & 1.0 & 79.1 \\
\hline \multirow[t]{3}{*}{ Debutyldronedarone } & 0.08 & 0.086 & 10.1 & 7.5 & 0.086 & 10.2 & 7.7 & 69.9 \\
\hline & 1 & 0.997 & 2.9 & -0.3 & 1.012 & 4.9 & 1.2 & 72.2 \\
\hline & 100 & 101.239 & 2.8 & 1.2 & 101.385 & 3.5 & 1.4 & 79.9 \\
\hline \multirow[t]{3}{*}{ Amiodarone } & 0.08 & 0.084 & 11.5 & 5.3 & 0.087 & 11.1 & 8.3 & 69.5 \\
\hline & 1 & 0.987 & 4.3 & -1.3 & 1.000 & 2.5 & 0.0 & 68.6 \\
\hline & 100 & 99.425 & 3.0 & -0.6 & 100.668 & 2.0 & 0.7 & 72.0 \\
\hline \multirow[t]{3}{*}{ Desethylamiodarone } & 0.08 & 0.087 & 9.7 & 6.2 & 0.079 & 13.3 & 7.8 & 68.7 \\
\hline & 1 & 1.014 & 2.7 & 1.4 & 0.980 & 5.2 & -2.0 & 71.2 \\
\hline & 100 & 99.518 & 3.7 & -0.5 & 100.437 & 2.5 & 0.4 & 75.5 \\
\hline
\end{tabular}




\section{CONCLUSION}

The proposed HPLC-UV method enables detection of dronedarone and debutyldronedarone in plasma and myocardial tissue, and has been validated with respect to linearity, accuracy, precision, recovery, limit of detection and lower limit of quantification. This method may be used for pharmacokinetic studies or for measuring dronedarone levels in patients for whom under- or overdosage is suspected.

In addition, the present assay has been validated for determination of amiodarone and desethylamiodarone with limits of detection for plasma samples comparable to the lowest limits of detection reported in previous HPLC-UV assays.,12-14 Moreover, this paper reports the lowest limits of detection for tissue samples, as other studies mainly showed validation data for plasma samples, but showed only limited data for tissue samples.

A 10-minute chromatographic runtime was used to validate the current method for both dronedarone and amiodarone (and principal metabolites). However, for selective determination of dronedarone and debutyldronedarone one may use desethylamiodarone as internal standard to achieve a runtime of less than 5 minutes. 


\section{REFERENCES}

1. Singh BN. Amiodarone as paradigm for developing new drugs for atrial fibrillation. J Cardiovasc Pharmacol. 2008;52(4):300-305.

2. Kodama I, Kamiya K, Toyama J. Cellular electropharmacology of amiodarone. Cardiovasc Res. 1997;35(1):13-29.

3. Zimetbaum P. Amiodarone for atrial fibrillation. N Engl J Med. 2007;356(9):935-941.

4. Laughlin JC, Kowey PR. Dronedarone: a new treatment for atrial fibrillation. J Cardiovasc Electrophysiol. 2008;19(11):1220-6

5. Hohnloser SH, Crijns HJ, van Eickels M, et al. Effect of dronedarone on cardiovascular events in atrial fibrillation. $N$ Engl J Med. 2009;360(7):668-678.

6. Pollak PT. A systematic review and critical comparison of internal standards for the routine liquid chromatographic assay of amiodarone and desethylamiodarone. Ther Drug Monit. 1996;18(2):168-178.

7. Kollroser M, Schober C. Determination of amiodarone and desethylamiodarone in human plasma by high-performance liquid chromatography-electrospray ionization tandem mass spectrometry with an ion trap detector. J Chromatogr B Analyt Technol Biomed Life Sci. 2002;766(2):219-226.

8. Ha HR, Bigler L, Wendt B, et al. Identification and quantitation of novel metabolites of amiodarone in plasma of treated patients. Eur J Pharm Sci. 2005;24(4):271-279.

9. Maes A, Baert K, Croubels S, et al. Determination of amiodarone and desethylamiodarone in horse plasma and urine by high-performance liquid chromatography combined with UV detection and electrospray ionization mass spectrometry. J Chromatogr B Analyt Technol Biomed Life Sci. 2006;836(1-2):47-56.

10. Li S, Liu G, Jia J, et al. Simultaneous determination of ten antiarrhythic drugs and a metabolite in human plasma by liquid chromatography-tandem mass spectrometry. J Chromatogr B Analyt Technol Biomed Life Sci. 2007;847(2):174-181.

11. Perez-Ruiz T, Martinez-Lozano C, Garcia-Martinez MD. Simultaneous determination of amiodarone and its metabolite desethylamiodarone by high-performance liquid chromatography with chemiluminescent detection. Anal Chim Acta. 2008;623(1):89-95.

12. Plomp TA, Engels $M$, Robles de Medina EO, et al. Simultaneous determination of amiodarone and its major metabolite desethylamiodarone in plasma, urine and tissues by highperformance liquid chromatography. J Chromatogr. 1983;273(2):379-392.

13. Kannan R, Miller S, Perez V, et al. Sensitive method for the measurement of amiodarone and desethylamiodarone in serum and tissue and its application to disposition studies. J Chromatogr. 1987;385:225-232.

14. Moor MJ, Wyss PA, Bickel MH. New procedure for the high-performance liquid chromatographic determination of amiodarone and desethylamiodarone with solid-phase extraction of rat plasma and tissue samples. J Chromatogr. 1988;431(2):455-460. 


\section{CHAPTER 3}

\section{Intrapericardial delivery of amiodarone and sotalol: atrial transmural drug distribution and electrophysiological effects}

Robert W. Bolderman, J.J. Rob Hermans, Leonard M.F. Rademakers, Tim S. Jansen, Sander Verheule, Frederik H. van der Veen, Jos G. Maessen. Intrapericardial delivery of amiodarone and sotalol: atrial transmural drug distribution and electrophysiological effects.

J Cardiovasc Pharmacol 2009;54:355-363. 


\begin{abstract}
Amiodarone and sotalol are frequently used in the treatment of atrial fibrillation. However, oral and intravenous (IV) therapy with these drugs has suboptimal efficacy and is associated with serious extracardiac side effects. We hypothesized that intrapericardial (IPC) delivery produces antiarrhythmic effects at lower plasma drug concentrations than IV delivery. Goats $(n=27)$ were randomised into 5 groups receiving either IPC vehicle, amiodarone (IV or IPC) or DL-sotalol (IV or IPC). Epicardial and endocardial atrial effective refractory period and atrial response to burst pacing (rapid atrial response, RAR) were assessed before and after 3 hours of drug infusion at $2 \mathrm{mg} / \mathrm{kg} / \mathrm{h}$. IPC delivery produced steeply decreasing drug concentrations from epicardium to endocardium in both atria and ventricles. Plasma drug concentrations were significantly lower in IPC than in IV groups. IPC amiodarone and sotalol reduced epicardial RAR inducibility $(-74 \pm 20$ and $-66 \pm 30 \%$, respectively) compared with IV delivery $(-11 \pm 17$ and $-17 \pm 28 \%$, respectively; $P<0.05)$. Endocardial RAR inducibility was only reduced in the IPC amiodarone group $(-70 \pm 17 \%, P<0.05)$. In conclusion, IPC delivery of amiodarone and sotalol increases atrial drug concentration and antiarrhythmic effects at reduced plasma drug concentrations. These potential benefits are particularly prominent for IPC delivered amiodarone.
\end{abstract}




\section{INTRODUCTION}

Amiodarone and sotalol, both class III antiarrhythmic agents, are frequently used in the treatment of atrial fibrillation (AF). However, oral and intravenous (IV) therapy with these drugs has suboptimal efficacy and is associated with serious extracardiac side effects. ${ }^{1-3}$ Local drug delivery may overcome these problems by producing therapeutic atrial drug concentrations at low plasma drug concentrations. Because the atria are thin structures with a relatively large epicardial surface area, intrapericardial (IPC) drug delivery could be an effective way to target the atrial myocardium.

With recent developments in minimally invasive access to the pericardial cavity and long-term controlled drug release systems, IPC drug delivery has become clinically feasible. ${ }^{4-11}$ However, important questions regarding myocardial drug distribution and electrophysiological effects during IPC drug delivery remain unanswered. Especially, the presence of transmural drug concentration gradients during IPC drug delivery complicates assessment of antiarrhythmic effects. ${ }^{12-16}$ Transmural drug concentration gradients may even enhance arrhythmogenic electrophysiological transmural dispersion, although previous IPC drug delivery studies did not show increased proarrhythmia.

In a recent study in our laboratory, we showed that transmural drug concentration gradients during IPC drug delivery are not only present in the ventricles but also in the atria and this produces dissimilar effects on epicardial and endocardial atrial electrophysiology. ${ }^{16}$ The study compared efficacy of IPC and IV delivered sotalol and flecainide to reduce the number of cardioversions in goats with pacinginduced sustained AF, but a benefit of IPC drug delivery to terminate AF could not be demonstrated. Regarding amiodarone, IPC infusion has been shown to suppress electrically induced AF. ${ }^{12}$ However, only mean atrial drug concentrations and only epicardial electrophysiological effects were measured. Furthermore, efficacy of IPC amiodarone delivery has never been compared with IV delivery.

To the best of our knowledge, atrial transmural concentration gradients and atrial transmural electrophysiology during IPC delivery of amiodarone have not been studied so far. In addition, IPC delivery of amiodarone and sotalol, the antiarrhythmic agents most frequently used for sinus rhythm maintenance, has never been compared. Because both drugs differ in molecular weight, lipophilicity, and electrical charge, one would expect differences in transmural drug distribution during IPC delivery, resulting in differences between epicardial and endocardial electrophysiological effects and differences between plasma drug levels.

We hypothesized that IPC delivery produces antiarrhythmic effects at lower plasma drug concentrations than IV delivery. Our study aimed to compare transmural myocardial and plasma drug concentrations after IPC and IV delivery of 
amiodarone and sotalol. In addition, epicardial and endocardial electrophysiological parameters were studied in the right atrium and right ventricle of goats.

\section{METHODS}

All procedures conformed to the Guide for the Care and Use of Laboratory Animals published by the National Institutes of Health (NIH publication No. 85-23, revised 1996). In addition, the Animal Studies Committee of the University of Maastricht approved this study protocol.

Domestic goats ( $\mathrm{n}=27$, weight $46-75 \mathrm{~kg}$ ) were randomized into 5 groups: control $(\mathrm{n}=5)$, IV amiodarone (IV AM, n=5), IV sotalol (IV SO, n=5), IPC amiodarone (IPC AM, n=6), and IPC sotalol (IPC SO, n=6).

\section{Surgical Procedure}

All animals were anesthetized by thiopental $20 \mathrm{mg} / \mathrm{kg}$ IV and maintained on isoflurane $(2 \%-3 \%)$ with a $1: 1$ mixture of oxygen and air. Analgesia consisted of buprenorphine $(10 \mu \mathrm{g} / \mathrm{kg} \mathrm{IV})$. Limb-lead electrocardiogram, end-expiratory $\mathrm{CO}_{2}$, and oxygen saturation (pulse oximeter) were monitored throughout the procedure. An external heating pad was used to maintain a rectal temperature of $38-39{ }^{\circ} \mathrm{C}$. Saline $(0.9 \%)$ was administered via a peripheral venous catheter at $5-8 \mathrm{mg} / \mathrm{kg} / \mathrm{h}$.

The right carotid artery was cannulated with a catheter attached to a pressure transducer for measuring systolic and diastolic arterial pressures. A right jugular venotomy was performed to insert 2 quadripolar steerable ablation catheters (2.5$\mathrm{mm}$ interelectrode distance) and 2 single-lumen catheters for IV drug delivery and coronary sinus sampling.

After a right intercostal thoracotomy, short incisions $(2 \mathrm{~mm})$ were made in the pericardium above the free walls of right atrium and ventricle. A single-lumen catheter for IPC drug delivery and 2 quadripolar steerable ablation catheters (2.5$\mathrm{mm}$ interelectrode distance) were inserted into the pericardial cavity. Catheter insertion sites were closed with a purse-string suture to prevent pericardial fluid leakage. The tip of the IPC infusion catheter was positioned above the lateral free wall of the right atrium. Under fluoroscopic guidance, endocardial and epicardial quadripolar catheters were positioned opposite to each other on the lateral free walls of the right atrium and ventricle.

\section{Electrophysiological Measurements}

Electrocardiogram limb leads and quadripolar catheters were connected to an electrophysiology monitoring system with integrated amplifier/stimulator (EP-Tracer 
38; CardioTek, Maastricht-Airport, the Netherlands). RR, PQ, QT, and maximal $\mathrm{T}_{\text {peak }}-\mathrm{T}_{\text {end }}$ intervals and $\mathrm{P}$ and QRS widths were measured during normal sinus rhythm. QT interval was calculated using the Bazett correction formula. Eight bipolar electrograms were recorded simultaneously at 4 sites: epicardial and endocardial right atrial free wall and epicardial and endocardial right ventricular free wall. All animals underwent electrophysiological testing at baseline and after 60, 120, and 180 minutes of infusion with drug solutions and vehicle.

Atrial and ventricular effective refractory periods (AERP and VERP) were measured during bipolar pacing at 4 times the threshold. Ten basic stimuli (S1) with a 400-millisecond interval were followed by an extrastimulus (S2). The S1-S2 interval was incremented in 2-millisecond steps and the longest S1-S2 interval failing to capture defined the effective refractory period (ERP). Atrial and ventricular transmural dispersion of refractoriness (TDR) were calculated by subtracting epicardial from endocardial ERP measured by oppositely positioned epicardial and endocardial catheters.

Epicardial and endocardial atrial and ventricular conduction times were measured during 400-millisecond interval pacing with the distal electrode of each catheter. Conduction time was defined as time between stimulus artifact and corresponding atrial electrogram in the proximal electrode.

To assess atrial vulnerability to arrhythmia, 10 basic stimuli (S1) with 400millisecond intervals were applied, followed by 1 second of $50-\mathrm{Hz}$ burst pacing (S2). A rapid atrial response (RAR) was considered inducible if a rapid irregular rhythm lasting $>1$ second occurred after S2. Epicardial and endocardial RAR inducibility were quantified by the percentage of positive inductions after 20 attempts. Mean and median RAR durations were calculated from induced RAR episodes.

\section{Drug Infusion Protocol}

To a standard IV amiodarone solution (Sanofi-Synthélabo, Maassluis, the Netherlands), containing $50 \mathrm{mg} / \mathrm{ml}$ amiodarone, $100 \mathrm{mg} / \mathrm{ml}$ polysorbate 80 , and $20.2 \mathrm{mg} / \mathrm{ml}$ of benzyl alcohol, we added $20 \% \mathrm{vol} / \mathrm{vol}$ polysorbate 80 and $4 \% \mathrm{vol} / \mathrm{vol}$ benzyl alcohol to improve amiodarone solubility. This was diluted in $5 \%$ dextrose and $4.2 \%$ sodium bicarbonate to obtain a drug concentration of $6 \mathrm{mg} / \mathrm{ml}$ at $\mathrm{pH}$ 7.4. A standard $10 \mathrm{mg} / \mathrm{ml}$ IV DL-sotalol solution (Bristol-Myers Squibb, Woerden, the Netherlands) was similarly diluted to obtain a drug concentration of $6 \mathrm{mg} / \mathrm{ml}, \mathrm{pH} \mathrm{7.4}$, and equal concentrations of polysorbate 80 and benzyl alcohol to the amiodarone solution. Control animals received vehicle, containing equal concentrations of polysorbate 80 and benzyl alcohol to the drug solutions.

The drug solutions were infused for 3 hours at a constant rate of $2 \mathrm{mg} / \mathrm{kg} / \mathrm{h}$ (infusion volume $0.33 \mathrm{mg} / \mathrm{kg} / \mathrm{h}$ ). This drug infusion protocol was selected based on 
a previous study on IPC SO delivery, which demonstrated an increase in AF cycle length during a similar infusion protocol. ${ }^{16}$ To determine an equipotent dose of amiodarone, pilot experiments were performed that showed similar increases in endocardial AERP for equal amiodarone and sotalol doses of $2 \mathrm{mg} / \mathrm{kg} / \mathrm{h}$ for 3 hours. These amiodarone and sotalol doses are in the range of clinically applied IV doses. ${ }^{17-19}$ To compensate for the electrophysiological and hemodynamic effects of the solvents and IPC volume expansion, IV drug-infused animals received IPC vehicle infusion, whereas IPC drug-infused animals received IV vehicle infusion. Control animals received both IPC and IV vehicle infusion.

\section{Drug Concentration Measurements}

Blood samples were drawn from a peripheral vein of the hind limb and from the coronary sinus before infusion and after 60, 120, and 180 minutes of infusion with drug solutions. The blood was centrifuged, and the plasma was stored at $-80{ }^{\circ} \mathrm{C}$. After completion of the electrophysiological measurements, the heart was excised. Of every heart, 4 free-wall sections of $4 \mathrm{~cm}^{2}$ were dissected from both atria and both ventricles. These were frozen in liquid nitrogen and stored at $-80{ }^{\circ} \mathrm{C}$. To assess transmural drug concentration gradients, free-wall sections were freeze microtomed parallel to the epicardial surface in slices of $500 \mu \mathrm{m}$. Drug concentrations in plasma and tissue were determined by high-performance liquid chromatography coupled with fluorescence and UV detection for sotalol and amiodarone, respectively. ${ }^{20,21}$

\section{Statistical Analysis}

Data are expressed as mean \pm SD. Multigroup comparisons were made by a KruskalWallis test. For individual comparisons, between- and within-group effects were calculated by Mann-Whitney $U$ and Wilcoxon signed rank tests, respectively. A 2tailed $P$ value of $<0.05$ was considered statistically significant.

\section{RESULTS}

All animals were in normal sinus rhythm at the beginning of the study and were hemodynamically stable during the entire procedure. Epicardial and endocardial atrial and ventricular pacing thresholds did not significantly change in any group during the course of the experiments. 


\section{Drug Distribution}

IV drug delivery resulted in homogeneous transmural drug distribution with overall mean atrial amiodarone and sotalol concentrations of $12 \pm 4$ and $18 \pm 4 \mu \mathrm{g} / \mathrm{g}$, respectively (Figure 1). In contrast, IPC AM and IPC SO delivery produced a steep transmural drug concentration gradient ranging from $341 \pm 61$ and $245 \pm 52 \mu \mathrm{g} / \mathrm{g}$ at the epicardial side of the atrium to $17 \pm 10$ and $21 \pm 10 \mu \mathrm{g} / \mathrm{g}$ at the endocardial side of the atrium for amiodarone and sotalol, respectively. Ventricular drug concentrations were similar to atrial concentrations in all groups, except for IPC AM. IPC AM produced significantly lower ventricular drug concentrations at $1.5 \mathrm{~mm}$ and farther from the epicardium compared with IV delivery.

Plasma concentrations of both amiodarone and sotalol were significantly lower during IPC drug infusion than during IV drug infusion (Table 1). Differences in plasma levels were particularly prominent for the amiodarone groups, with $53 \pm 17$ times lower peripheral plasma levels in the IPC group after 3 hours of drug infusion. In the IV drug groups, plasma levels appeared to be already constant after 1 hour of drug infusion, whereas after 3 hours of IPC drug delivery, plasma levels were still climbing. Although the coronary sinus in goats drains blood not only from the myocardial tissue but also from the left azygos vein, plasma drug concentrations in the coronary sinus were significantly higher than peripheral plasma concentrations even after 3 hours of IPC delivery of both amiodarone and sotalol.

Table 1. Plasma drug distribution

\begin{tabular}{|c|c|c|c|c|c|c|c|c|}
\hline \multirow{2}{*}{$\begin{array}{l}\text { Minutes after start } \\
\text { of drug infusion }\end{array}$} & \multicolumn{2}{|c|}{ IV Amiodarone } & \multicolumn{2}{|c|}{ IPC Amiodarone } & \multicolumn{2}{|c|}{ IV Sotalol } & \multicolumn{2}{|c|}{ IPC Sotalol } \\
\hline & PV & CS & PV & CS & PV & CS & $\mathrm{PV}$ & $\mathrm{CS}$ \\
\hline $60 \mathrm{~min}$ & $1.3 \pm 0.2$ & $1.3 \pm 0.2$ & $0.015 \pm 0.005^{*}$ & $0.034 \pm 0.006^{*} \dagger$ & $8.3 \pm 0.8$ & $7.9 \pm 0.5$ & $1.2 \pm 0.3^{*}$ & $3.8 \pm 0.6^{*}+$ \\
\hline $120 \mathrm{~min}$ & $1.4 \pm 0.2$ & $1.5 \pm 0.2$ & $0.019 \pm 0.004^{*}$ & $0.056 \pm 0.014^{*} \dagger$ & $7.4 \pm 0.9$ & $7.2 \pm 0.5$ & $3.1 \pm 0.3^{*}$ & $5.1 \pm 0.5^{*}+$ \\
\hline $180 \mathrm{~min}$ & $1.4 \pm 0.2$ & $1.5 \pm 0.2$ & $0.027 \pm 0.004^{*}$ & $0.066 \pm 0.009^{*} \dagger$ & $8.5 \pm 0.9$ & $8.1 \pm 0.6$ & $3.9 \pm 0.1^{*}$ & $5.5 \pm 0.3^{*}+$ \\
\hline
\end{tabular}

PV indicates peripheral vein; SC, coronary sinus. Plasma drug concentrations in $\mu g / m l$. Values are mean \pm SD. $n=5$ for intravenous drug groups and $n=6$ for intrapericardial drug groups. ${ }^{*} \mathrm{P}<0.05$ vs $I V$; $+\mathrm{P}<0.05$ vs $\mathrm{PV}$. 

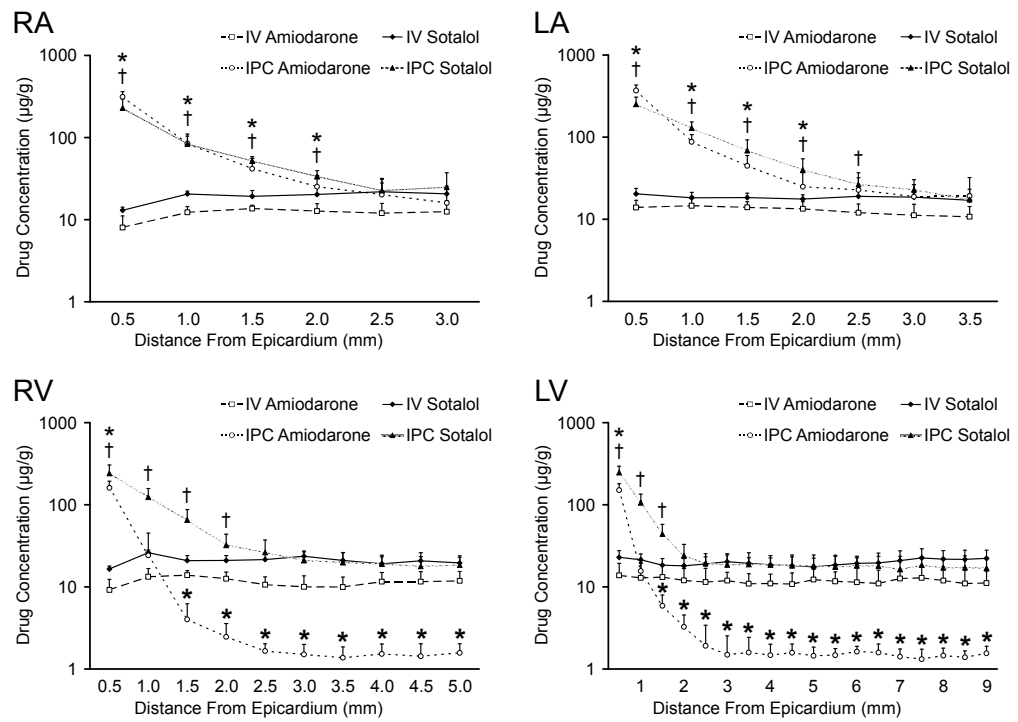

Figure 1. Transmural myocardial drug distribution after 3 hours of drug infusion. RA indicates right atrium; LA, left atrium; RV, right ventricle; $L V$, left ventricle. Values are mean \pm SD. $n=5$ for intravenous drug groups and $n=6$ for intrapericardial drug groups. ${ }^{*} \mathrm{P}<0.05$ IPC vs IV Amiodarone. $+\mathrm{P}<0.05$ IPC vs IV Sotalol.

\section{Electrophysiological Findings}

\section{Atrial Refractoriness and Conduction}

Effects of IPC and IV drug infusion on atrial epicardial and endocardial ERP are shown in Figure 2. The very high epicardial drug concentrations during IPC infusion produced a marked prolongation of epicardial ERP in the IPC SO group, whereas IPC infused amiodarone produced a smaller increase in epicardial ERP. Endocardial drug concentrations did not differ between IPC and IV groups, which was reflected by ERP increases of similar magnitude. In IV drug groups, the equal increases in epicardial and endocardial ERP caused no change in atrial TDR. However, because of the relatively larger increase in epicardial ERP, IPC drug infusion produced a decrease in atrial TDR, which, in case of IPC SO, even led to an inversion of atrial TDR.

No significant differences were found between epicardial and endocardial atrial conduction times in any group at a given time point. Mean conduction times increased in all groups, ranging from $4.7 \%$ increase in control group to $9.3 \%-10.5 \%$ increase in drug groups compared with baseline values, without significant differences between drug groups. 

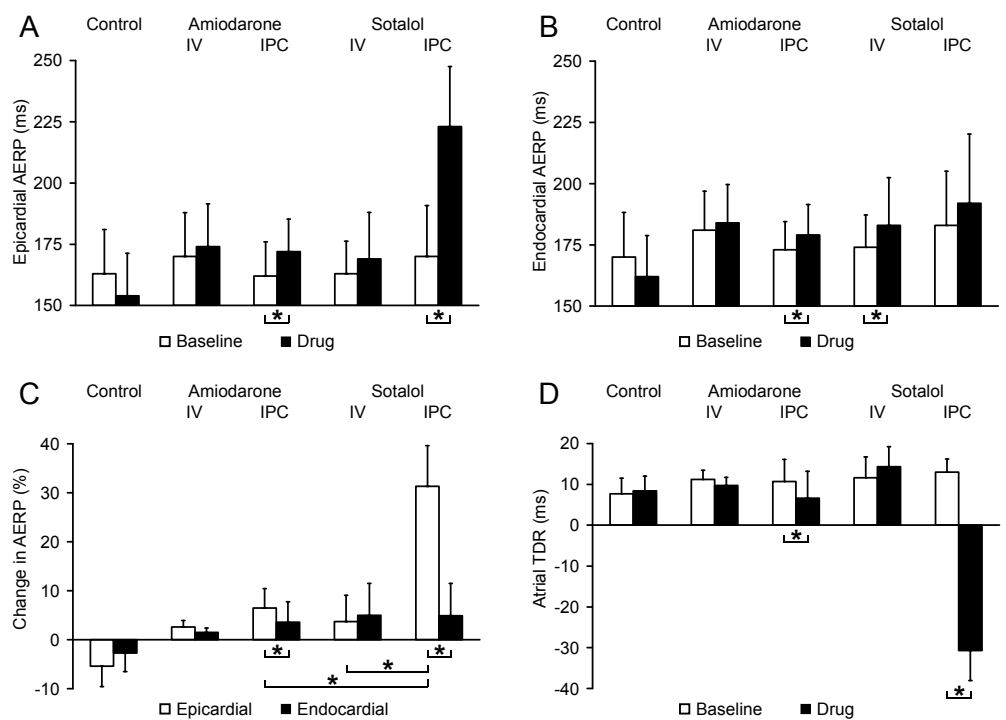

Figure 2. Atrial refractoriness at baseline and after 3 hours of drug infusion. A, Epicardial AERP. B, Endocardial AERP. C, Change in epicardial and endocardial AERP after 3 hours of drug infusion relative to baseline measurements. D, Atrial TDR. Values are mean \pm SD. $n=5$ for control and intravenous drug groups and $n=6$ for intrapericardial drug groups. ${ }^{*} P<0.05$.
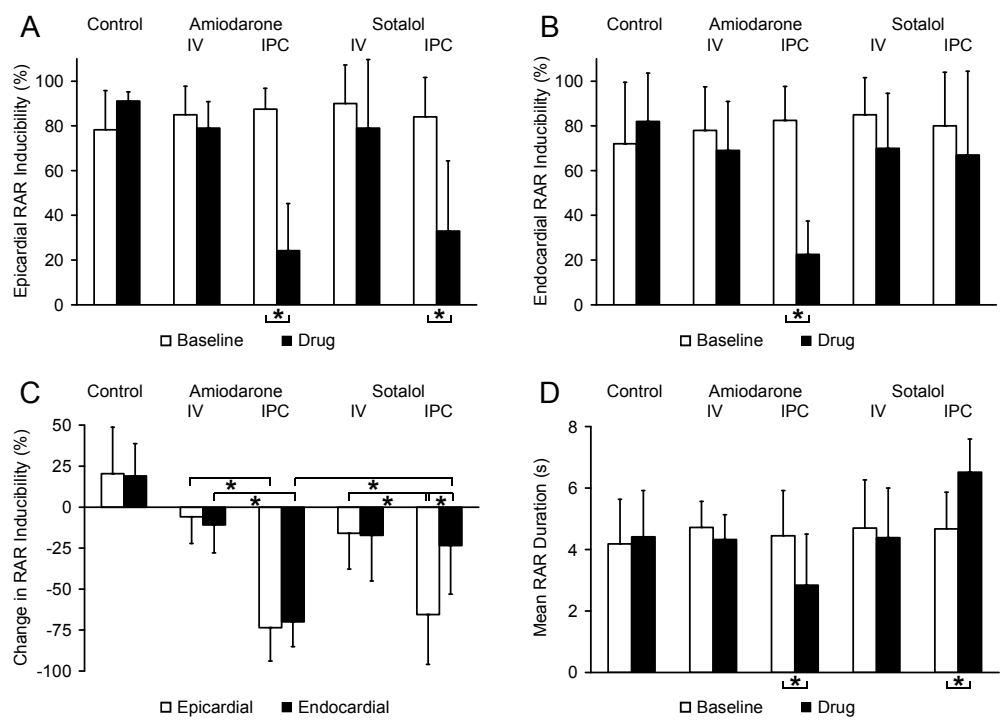

Figure 3. Vulnerability to atrial arrhythmia at baseline and after 3 hours of drug infusion. A, Epicardial rapid atrial response (RAR) inducibility. B, Endocardial RAR inducibility. C, Change in epicardial and endocardial RAR inducibility after 3 hours of drug infusion relative to baseline measurements. D, Mean RAR duration. Values are mean \pm SD. $n=5$ for control and intravenous drug groups and $n=6$ for intrapericardial drug groups. ${ }^{*} P<0.05$. 


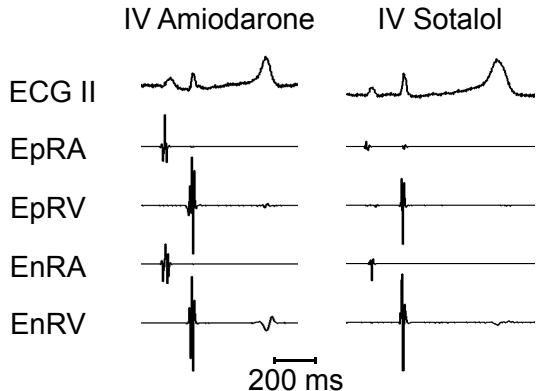

IPC Amiodarone IPC Sotalol

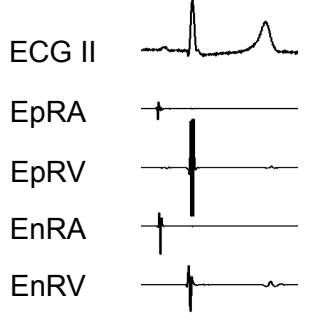

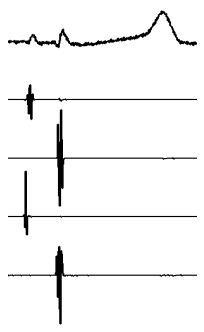

Figure 4. Typical electrograms after 3 hours of drug infusion. Ep indicates epicardial; En, endocardial; RA, right atrial; RV, right ventricular distal electrode.

Table 2. Hemodynamic and ECG parameters before and after 3 hours of drug infusion

\begin{tabular}{|c|c|c|c|c|c|c|c|c|c|c|}
\hline & \multicolumn{2}{|c|}{ Control } & \multicolumn{2}{|c|}{ IV Amiodarone } & \multicolumn{2}{|c|}{ IPC Amiodarone } & \multicolumn{2}{|c|}{ IV Sotalol } & \multicolumn{2}{|c|}{ IPC Sotalol } \\
\hline & Baseline & $\overline{\text { Vehicle }}$ & Baseline & Drug & Baseline & Drug & Baseline & Drug & Baseline & Drug \\
\hline $\mathrm{MABP}, \mathrm{mmHg}$ & $74 \pm 13$ & $62 \pm 17^{*}$ & $72 \pm 11$ & $56 \pm 11^{*}$ & $75 \pm 6$ & $64 \pm 11^{*}$ & $68 \pm 9$ & $46 \pm 11^{*}$ & $74 \pm 11$ & $49 \pm 9^{*}$ \\
\hline RR interval, ms & $698 \pm 147$ & $720 \pm 161$ & $701 \pm 132$ & $769 \pm 88$ & $651 \pm 94$ & $762 \pm 83^{*}$ & $664 \pm 54$ & $925 \pm 105^{*}$ & $632 \pm 91$ & $1146 \pm 44^{*}$ \\
\hline PQ interval, ms & $132 \pm 19$ & $134 \pm 15$ & $122 \pm 24$ & $130 \pm 22$ & $128 \pm 23$ & $136 \pm 24$ & $129 \pm 28$ & $156 \pm 25^{*}$ & $128 \pm 19$ & $154 \pm 24^{*}$ \\
\hline QT interval, ms & $401 \pm 40$ & $416 \pm 35$ & $400 \pm 42$ & $430 \pm 42$ & $389 \pm 34$ & $431 \pm 46^{*}$ & $409 \pm 27$ & $503 \pm 42^{*}$ & $386 \pm 39$ & $575 \pm 32^{*}$ \\
\hline $\mathrm{QT}_{\mathrm{c}}$ interval, $\mathrm{ms}$ & $483 \pm 21$ & $494 \pm 26$ & $479 \pm 28$ & $491 \pm 26$ & $484 \pm 26$ & $495 \pm 36$ & $503 \pm 45$ & $523 \pm 26$ & $486 \pm 26$ & $538 \pm 34^{*}$ \\
\hline $\max \mathrm{T}_{\text {peak-end, }} \mathrm{ms}$ & $50 \pm 10$ & $56 \pm 6$ & $53 \pm 10$ & $66 \pm 11^{*}$ & $54 \pm 9$ & $64 \pm 8^{*}$ & $51 \pm 11$ & $69 \pm 21^{*}$ & $54 \pm 16$ & $81 \pm 20^{*}$ \\
\hline P width, ms & $52 \pm 9$ & $54 \pm 11$ & $57 \pm 13$ & $58 \pm 11$ & $55 \pm 13$ & $56 \pm 13$ & $60 \pm 5$ & $65 \pm 7$ & $57 \pm 8$ & $62 \pm 8$ \\
\hline QRS width, ms & $58 \pm 7$ & $60 \pm 7$ & $60 \pm 6$ & $61 \pm 4$ & $57 \pm 9$ & $60 \pm 11$ & $58 \pm 8$ & $60 \pm 7$ & $57 \pm 8$ & $58 \pm 7$ \\
\hline
\end{tabular}

MABP indicates mean arterial blood pressure; max $\mathrm{T}_{\text {peak-end, }}$ maximal $\mathrm{T}_{\text {peak }}-\mathrm{T}_{\text {end }}$ interval. Presented data were obtained from ECG recordings during normal sinus rhythm. Values are mean $\pm S D$. $n=5$ for control and intravenous drug groups and $n=6$ for intrapericardial drug groups. ${ }^{*} P<0.05$ vs baseline.

Vulnerability to Atrial Arrhythmia

Before drug infusion, RAR episodes of more than 1 second could readily be induced in all animals by burst pacing at both the right atrial epicardium and endocardium (Figure 3). After 3 hours of IPC drug infusion, the very high epicardial drug concen- 
trations produced a significant decrease in epicardial RAR inducibility for both drugs. On the endocardial side, a similar decrease in RAR inducibility was only observed in the IPC AM group.

As there were no differences in duration between epicardially and endocardially induced RAR episodes, irrespective of treatment or treatment duration, RAR duration is given as mean of epicardially and endocardially induced RAR episodes (Figure 3D). In line with a reduction in epicardial and endocardial RAR inducibility, IPC AM produced a shortening of mean RAR duration. Although IPC SO decreased epicardial RAR inducibility, mean RAR duration increased.

\section{Ventricular Electrophysiology}

Changes in right ventricular refractoriness and conduction time after drug delivery were comparable to those observed in the right atrium. In the IPC SO group, endocardial VERP was $6 \pm 3$ millisecond longer than epicardial VERP at baseline, but after 3 hours of drug infusion, epicardial VERP was $29 \pm 20$ millisecond longer than endocardial VERP. IPC delivery of amiodarone produced a more homogeneous prolongation of epicardial and endocardial VERP, reducing ventricular TDR from $7 \pm 5$ millisecond at baseline to $3 \pm 3$ millisecond after 3 hours of drug infusion. No ST segment changes, T-wave inversions, or ventricular arrhythmias were observed. However, IPC SO produced larger increases in RR, QT, QT $c_{c}$, and maximal $\mathrm{T}_{\text {peak }}-\mathrm{T}_{\text {end }}$ intervals than IV sotalol (Table 2 and Figure 4).

\section{Discussion}

The rationale of IPC drug delivery is that therapeutic myocardial concentrations can be achieved, while systemic drug concentrations can be reduced. This has been demonstrated for various antiarrhythmic substances, including lidocaine, ${ }^{22,23}$ digoxin, ${ }^{22,24}$ amiodarone, ${ }^{12,13}$ l-arginine, ${ }^{25}$ esmolol, ${ }^{26}$ procainamide, ${ }^{14,24}$ nitroglycerin, ${ }^{27}$ ibutilide,,$^{15}$ sotalol, ${ }^{16,20}$ atenolol, ${ }^{20}$ docosahexaenoic acid, ${ }^{28}$ and flecainide. ${ }^{16}$ However, until recently, it was unknown that IPC delivery of antiarrhythmic drugs produces transmural concentration gradients in the atria, resulting in differences between epicardial and endocardial atrial electrophysiology. ${ }^{16}$

The present study, for the first time, compared atrial transmural concentration gradients and transmural electrophysiology after IPC delivery of 2 antiarrhythmic drugs with distinct differences in physicochemical properties; amiodarone has a higher molecular weight, is highly lipophilic, and, at $\mathrm{pH}$ 7.4, is less positively charged than sotalol. Previous studies on IPC AM showed high atrial drug concentrations, but only mean concentrations were measured.12,13 A recent study in our laboratory demonstrated that IPC SO produces very high epicardial atrial drug 
concentrations and lower endocardial drug concentrations, ${ }^{16}$ which is consistent with results of the present study.

Interestingly, the present study additionally shows that amiodarone is similarly distributed across both atrial walls after IPC delivery. Whether this is due to the fact that the diffusion characteristics in the atria are similar for both amiodarone and sotalol, despite their physicochemical differences, or whether the drugs may also migrate through the myocardium by a different mechanism than plain diffusion, for example, convection via microcirculation or lymphatics, is unclear. A second interesting finding is that IPC delivery of amiodarone produced much steeper ventricular transmural drug concentration gradients than IPC SO delivery. IPC AM even produced lower ventricular drug concentrations compared with IV delivery at 1.5 $\mathrm{mm}$ and farther from the epicardium. This may be due to different ventricular diffusion or perfusion characteristics between amiodarone and sotalol.

Despite similar atrial transmural drug concentration gradients, IPC delivery of amiodarone and sotalol produced dissimilar changes in atrial transmural electrophysiology. For sotalol, atrial tissue concentrations and electrophysiological effects showed a fairly consistent relationship. However, during IPC delivery of amiodarone, the increase in endocardial AERP was approximately $50 \%$ of the epicardial AERP increase, despite much lower endocardial amiodarone concentrations. Because epicardial concentrations of both amiodarone and sotalol were very high, it can be assumed that effects on epicardial AERP were maximal for both drugs. Therefore, it can be concluded that endocardial amiodarone concentrations produced already half-maximal effects on endocardial AERP. This is consistent with a previous study on IPC delivery of amiodarone, which demonstrated similar effects on epicardial and endocardial ERP in the right ventricle, despite 10-fold drug concentration differences between epicardium and endocardium. ${ }^{12}$

Another contributing factor for the inconsistent relationship between transmural amiodarone concentrations and effects on epicardial and endocardial electrophysiology might be transmural heterogeneity in ionic currents. Both amiodarone and sotalol possess $I_{\mathrm{Kr}}$ and $\beta$-blocking properties, but in addition, amiodarone blocks other potassium currents, including $I_{\mathrm{Ks}}, I_{\mathrm{K} 1}, I_{\mathrm{Kur}}, I_{\mathrm{to}}$, and $I_{\mathrm{KAch}}$, and sodium and calcium currents. ${ }^{29}$ Recent studies have shown that transmural heterogeneity in ionic currents is not only present in ventricles but also in atria. ${ }^{30-33}$ Therefore, the net epicardial or endocardial atrial effect of the drug does not only depend on the local drug concentration but also on the local density of ionic currents that are blocked by the drug. This may explain the reduction in atrial TDR that was observed during IPC AM delivery, despite a steep atrial transmural drug concentration gradient. In turn, a reduction in atrial TDR may explain the superior efficacy of IPC AM in reducing atrial vulnerability to arrhythmia. This is consistent with results of a previous study on IPC AM that demonstrated a reduction in epicardial AF inducibility. ${ }^{12}$ 
Efficacy has also been reported for IPC procainamide; a low dose produced changes in endocardial AERP and AF inducibility similar to a 10 times higher IV dose. ${ }^{14}$ The significant inversion of atrial TDR by IPC SO observed in the present study may explain the increase in arrhythmia duration. This is in line with the disappointing antiarrhythmic efficacy of IPC SO previously reported ${ }^{16}$ and could also explain similar AF termination rates between IPC ibutilide and control in dogs. ${ }^{15}$

Although previous studies on IPC AM showed low plasma levels after IPC delivery of amiodarone, this was compared with IV delivery up to 60 minutes after bolus injection. ${ }^{12,13}$ The present study demonstrates a significant pharmacokinetic advantage for IPC AM up to 3 hours of drug infusion at a constant rate. In addition, results show that the pharmacokinetic advantage is far greater for IPC AM than for IPC SO. The very low plasma concentrations after IPC delivery of amiodarone, in contrast to sotalol, are likely a result of slow diffusion of amiodarone to the bloodstream, followed by rapid distribution from the bloodstream into other tissues, which is consistent with the very large volume of distribution of amiodarone. ${ }^{34}$ Plasma levels of amiodarone and sotalol were still climbing after 3 hours of IPC drug delivery, whereas plasma levels appeared to be already constant after 1 hour of IV infusion. Therefore, pharmacokinetic advantages of IPC delivery would be expected to be lower at true steady state. However, a previous study demonstrated a similar 50\% reduction of sotalol plasma levels during IPC delivery compared with IV delivery at steady state. ${ }^{20}$ Pharmacokinetic advantages of IPC delivery during steady state conditions were also demonstrated for steroids and macromolecules. ${ }^{35}$ This was shown to be a result of low pericardial fluid clearances compared with relatively high plasma clearances. Although no data exist on steady state conditions during IPC delivery of amiodarone, the low amiodarone plasma concentrations after 3 hours suggest low pericardial fluid clearances. Therefore, it would take much longer to reach steady state plasma drug concentrations than after IPC delivery of sotalol. For future applications, one could make use of this property of amiodarone by performing intermittent IPC infusions to maintain the greatest advantage of local application.

IPC SO, in contrast to amiodarone, produced a markedly greater increase in QT interval than IV delivery. This is a point of concern because QT-interval prolongation may indicate an increased risk of ventricular arrhythmias. In a previous study in our laboratory, ${ }^{16}$ IV and IPC SO did not produce significant increases in QT interval in goats but QT-interval measurements were performed during a drug infusion protocol with lower sotalol dose and shorter infusion duration. IPC delivery of ibutilide did not change QT interval, ${ }^{15}$ whereas IPC procainamide and digoxin increased and decreased $\mathrm{QT}_{\mathrm{c}}$ interval, respectively. ${ }^{24}$ IPC AM in a previous study affected epicardial and endocardial electrophysiology of the right ventricle, whereas for the left ventricle only epicardial electrophysiology was affected. ${ }^{12}$ IPC procaina- 
mide produced no changes in global or endocardial ventricular electrophysiology, nor was it associated with ventricular proarrhythmia. ${ }^{14}$ The larger increase in QT interval by IPC SO observed in the present study can be explained by 2 factors. First, sotalol concentrations were very high in the epicardial layers of both ventricles. This is consistent with the large increase in epicardial ERP measured at the right ventricle in IPC SO treated goats. In contrast, transmural drug concentration gradients were much steeper and epicardial VERP increases were less pronounced after IPC delivery of amiodarone. Second, the larger increase in QT interval by IPC SO can be explained by the greater increase in RR interval due to higher sotalol concentrations in the superficial sinus node. The $T_{\text {peak }}-T_{\text {end }}$ interval divided by the QT interval may be a more accurate predictor for increased risk of ventricular arrhythmias. ${ }^{36}$ For IPC and IV delivery of both amiodarone and sotalol, these indices did not significantly differ, suggesting that risk of ventricular arrhythmias is not markedly higher for IPC delivery. However, because inducibility of ventricular fibrillation was not assessed, definite conclusions cannot be drawn.

Potential clinical applications of IPC delivered amiodarone and sotalol could involve prevention of AF after cardiac surgery or treatment of paroxysmal AF. Regarding the latter application, minimally invasive access to the normal pericardial cavity is required for clinical feasibility. Several promising concepts, including subxiphoid and transatrial approaches, ${ }^{4,5}$ were developed. Minimally invasive pericardial access may also facilitate a combination with other therapeutic approaches, such as epicardial mapping and catheter ablation of arrhythmias, and epicardial pacing. ${ }^{6}$ For prolonged IPC drug therapy, appropriate drug delivery systems with optimal biocompatibility and controlled drug release, for example, long-term IPC catheterization and drug releasing matrices, ${ }^{7,9-11}$ are needed. However, safety and efficacy of chronic IPC drug delivery should be the focus of further research.

\section{Limitations}

Nonremodeled atria of healthy goats were studied because sotalol has only moderate effects on electrically remodeled atria in chronic $\mathrm{AF}^{37}$ and drug diffusion characteristics would likely be affected in a sterile pericarditis model. Because the goats of this study did not have a substrate for AF, the duration of pacing-induced arrhythmia episodes were short, generally lasting several seconds. Arrhythmia episodes lasting more than 1 second were evaluated in this study, whereas studies with diseased atria use longer cutoff values. Only 1 common dose for amiodarone and sotalol was used in the present study. Although a change in dose would probably not alter relative drug distributions, it could alter pharmacodynamic profiles. Furthermore, transmural drug distributions and electrophysiological effects were compared at only 1 time point, before steady state was reached in IPC drug groups. 
Although a pharmacokinetic advantage would still be expected at true steady state, it would probably be less pronounced. In addition, antiarrhythmic effects of acute amiodarone therapy differ from chronic amiodarone therapy. A reduction in blood pressure was observed in all groups, which may have been caused by IV-infused vehicle-induced vasodilatation, ${ }^{38}$ IPC volume expansion, or decreased cardiac contractility. However, these parameters were not evaluated in the present study.

\section{Conclusions}

IPC delivery of amiodarone and sotalol increases atrial drug concentration and antiarrhythmic effects at reduced plasma drug concentrations. These potential benefits are particularly prominent for IPC delivered amiodarone, offering a promising concept to reduce systemic side effects during treatment of AF. 


\section{REFERENCES}

1. Singh BN, Singh SN, Reda DJ, Tang XC, Lopez B, Harris CL, Fletcher RD, Sharma SC, Atwood JE, Jacobson AK, Lewis HD, Raisch DW, Ezekowitz MD. Amiodarone versus sotalol for atrial fibrillation. N Engl J Med. 2005;352(18):1861-72.

2. Lafuente-Lafuente C, Mouly S, Longas-Tejero MA, Bergmann JF. Antiarrhythmics for maintaining sinus rhythm after cardioversion of atrial fibrillation. Cochrane Database Syst Rev. 2007;(4):CD005049.

3. Camm AJ. Safety considerations in the pharmacological management of atrial fibrillation. Int J Cardiol. 2008;127(3):299-306.

4. Verrier RL, Waxman S, Lovett EG, Moreno R. Transatrial access to the normal pericardial space: a novel approach for diagnostic sampling, pericardiocentesis, and therapeutic interventions. Circulation. 1998;98(21):2331-3.

5. Maisch B, Ristic AD, Rupp H, Spodick DH. Pericardial access using the PerDUCER and flexible percutaneous pericardioscopy. Am J Cardiol. 2001;88(11):1323-6.

6. D'Avila A, Scanavacca M, Sosa E, Ruskin JN, Reddy VY. Pericardial anatomy for the interventional electrophysiologist. J Cardiovasc Electrophysiol. 2003;14(4):422-30.

7. Bartoli CR, Akiyama I, Godleski JJ, Verrier RL. Long-term pericardial catheterization is associated with minimum foreign-body response. Catheter Cardiovasc Interv. 2007;70(2):221-7.

8. Ota T, Patronik NA, Schwartzman D, Riviere CN, Zenati MA. Minimally invasive epicardial injections using a novel semiautonomous robotic device. Circulation. 2008;118(14 Suppl):S11520.

9. Avitall B, Hare J, Zander G, Bockoff C, Tchou P, Jazayeri M, Akhtar M. Iontophoretic transmyocardial drug delivery. A novel approach to antiarrhythmic drug therapy. Circulation. 1992;85(4):1582-93.

10. Labhasetwar V, Underwood T, Gallagher M, Murphy G, Langberg J, Levy RJ. Sotalol controlled-release systems for arrhythmias: in vitro characterization, in vivo drug disposition, and electrophysiologic effects. J Pharm Sci. 1994;83(2):156-64.

11. Labhasetwar V, Strickberger SA, Underwood T, Davis J, Levy RJ. Prevention of acute inducible atrial flutter in dogs by using an ibutilide-polymer-coated pacing electrode. J Cardiovasc Pharmacol. 1998;31(3):449-55.

12. Ayers GM, Rho TH, Ben-David J, Besch HR, Zipes DP. Amiodarone instilled into the canine pericardial sac migrates transmurally to produce electrophysiologic effects and suppress atrial fibrillation. J Cardiovasc Electrophysiol. 1996;7(8):713-21.

13. Darsinos JT, Karli JN, Samouilidou EC, Krumbholz B, Pistevos AC, Levis GM. Distribution of amiodarone in heart tissues following intrapericardial administration. Int J Clin Pharmacol Ther. 1999;37(6):301-6.

14. Ujhelyi MR, Hadsall KZ, Euler DE, Mehra R. Intrapericardial therapeutics: a pharmacodynamic and pharmacokinetic comparison between pericardial and intravenous procainamide delivery. J Cardiovasc Electrophysiol. 2002;13(6):605-11.

15. Vereckei A, Gorski JC, Ujhelyi M, Mehra R, Zipes DP. Intrapericardial ibutilide administration fails to terminate pacing-induced sustained atrial fibrillation in dogs. Cardiovasc Drugs Ther. 2004;18(4):269-77.

16. Van Brakel TJ, Hermans JJ, Accord RE, Schotten U, Smits JF, Allessie MA, Maessen JG. Effects of intrapericardial sotalol and flecainide on transmural atrial electrophysiology and atrial fibrillation. J Cardiovasc Electrophysiol. 2009;20(2):207-15. 
17. Hohnloser SH, Meinertz T, Dammbacher T, Steiert K, Jahnchen E, Zehender M, Fraedrich G, Just $\mathrm{H}$. Electrocardiographic and antiarrhythmic effects of intravenous amiodarone: results of a prospective, placebo-controlled study. Am Heart J. 1991;121(1 Pt 1):89-95.

18. Watt AH, Hutchings A, Stephens MR, Routledge PA. Plasma amiodarone concentrations during intravenous infusion. Br J Clin Pharmacol. 1986;21(5):525-8.

19. Dorian P, Newman D, Sheahan R, Tang A, Green M, Mitchell J. d-Sotalol decreases defibrillation energy requirements in humans: a novel indication for drug therapy. J Cardiovasc Electrophysiol. 1996;7(10):952-61.

20. Van Brakel TJ, Hermans JJ, Janssen BJ, van Essen H, Botterhuis N, Smits JF, Maessen JG. Intrapericardial delivery enhances cardiac effects of sotalol and atenolol. J Cardiovasc Pharmacol. 2004;44(1):50-6.

21. Bolderman RW, Hermans JJ, Maessen JG. Determination of the class III antiarrhythmic drugs dronedarone and amiodarone, and their principal metabolites in plasma and myocardium by high-performance liquid chromatography and UV-detection. J Chromatogr B Analyt Technol Biomed Life Sci. 2009;877(18-19):1727-31.

22. Darsinos JT, Samouilidou EC, Krumholz B, Kontoyanni M, Pistevos AK, Karli JN, Theodorakis MG, Levis GM, Moulopoulos SD. Distribution of lidocaine and digoxin in heart tissues and aorta following intrapericardial administration. Int $J$ Clin Pharmacol Ther Toxicol. 1993;31(12):611-5.

23. Takada M, Dohi S, Akamatsu S, Suzuki A. Effects of pericardial lidocaine on hemodynamic parameters and responses in dogs anesthetized with midazolam and fentanyl. J Cardiothorac Vasc Anesth. 2007;21(3):393-9.

24. Kolettis TM, Kazakos N, Katsouras CS, Niokou D, Pappa L, Koulouras V, Stefanou P, Seferiadis C, Malamou-Mitsi V, Michalis LK, Marselos M, Sideris DA. Intrapericardial drug delivery: pharmacologic properties and long-term safety in swine. Int J Cardiol. 2005;99(3):415-21.

25. Fei L, Baron AD, Henry DP, Zipes DP. Intrapericardial delivery of L-arginine reduces the increased severity of ventricular arrhythmias during sympathetic stimulation in dogs with acute coronary occlusion: nitric oxide modulates sympathetic effects on ventricular electrophysiological properties. Circulation. 1997;96(11):4044-9.

26. Moreno R, Waxman $S$, Rowe K, Verrier RL. Intrapericardial beta-adrenergic blockade with esmolol exerts a potent antitachycardic effect without depressing contractility. J Cardiovasc Pharmacol. 2000;36(6):722-7.

27. Kumar K, Nguyen K, Waxman S, Nearing BD, Wellenius GA, Zhao SX, Verrier RL. Potent antifibrillatory effects of intrapericardial nitroglycerin in the ischemic porcine heart. J Am Coll Cardiol. 2003;41(10):1831-7.

28. Xiao YF, Sigg DC, Ujhelyi MR, Wilhelm JJ, Richardson ES, Iaizzo PA. Pericardial delivery of omega-3 fatty acid: a novel approach to reducing myocardial infarct sizes and arrhythmias. Am J Physiol Heart Circ Physiol. 2008;294(5):H2212-8.

29. Kodama I, Kamiya K, Toyama J. Cellular electropharmacology of amiodarone. Cardiovasc Res. 1997;35(1):13-29.

30. Feng J, Yue L, Wang Z, Nattel S. Ionic mechanisms of regional action potential heterogeneity in the canine right atrium. Circ Res. 1998;83(5):541-51.

31. Anyukhovsky EP, Rosenshtraukh LV. Electrophysiological responses of canine atrial endocardium and epicardium to acetylcholine and 4-aminopyridine. Cardiovasc Res. 1999;43(2):364-70.

32. Burashnikov A, Mannava S, Antzelevitch C. Transmembrane action potential heterogeneity in the canine isolated arterially perfused right atrium: effect of $I_{\mathrm{Kr}}$ and $I_{\mathrm{Kur}} / I_{\mathrm{to}}$ block. Am J Physiol Heart Circ Physiol. 2004;286(6):H2393-400. 
33. Antzelevitch C. Role of spatial dispersion of repolarization in inherited and acquired sudden cardiac death syndromes. Am J Physiol Heart Circ Physiol. 2007;293(4):H2024-38.

34. Holt DW, Tucker GT, Jackson PR, Storey GC. Amiodarone pharmacokinetics. Am Heart J. 1983;106(4 Pt 2):840-7.

35. Hermans JJ, van Essen H, Struijker-Boudier HA, Johnson RM, Theeuwes F, Smits JF. Pharmacokinetic advantage of intrapericardially applied substances in the rat. J Pharmacol Exp Ther. 2002;301(2):672-8.

36. Gupta P, Patel C, Patel H, Narayanaswamy S, Malhotra B, Green JT, Yan GX. T(p-e)/QT ratio as an index of arrhythmogenesis. J Electrocardiol. 2008;41(6):567-74.

37. Tse HF, Lau CP. Electrophysiologic actions of dl-sotalol in patients with persistent atrial fibrillation. J Am Coll Cardiol. 2002;40(12):2150-5.

38. Munoz A, Karila P, Gallay P, Zettelmeier F, Messner P, Mery M, Grolleau R. A randomized hemodynamic comparison of intravenous amiodarone with and without Tween 80. Eur Heart J. 1988;9(2):142-8. 


\section{CHAPTER 4}

\section{Epicardial application of an amiodarone- releasing hydrogel to suppress atrial tachyarrhythmias}




\section{ABSTRACT}

Background: Amiodarone is currently the most effective antiarrhythmic drug for sinus rhythm maintenance. However, due to serious extracardiac adverse effects, prophylactic amiodarone therapy is only appropriate for patients at high risk for postoperative atrial fibrillation (AF). We hypothesized that epicardial application of an amiodarone-releasing hydrogel would produce therapeutic myocardial drug concentrations, while systemic levels would remain low.

Methods: Goats were fitted with right atrial epicardial patch electrodes. A poly(ethylene glycol)-based hydrogel with amiodarone (1 $\mathrm{mg} / \mathrm{kg}$ bodyweight) $(n=10)$ or without drug $(n=6)$ was applied to the right atrial epicardium. Atrial effective refractory period (AERP), conduction time and atrial response to burst pacing (rapid atrial response, RAR) were assessed up to 28 days in awake goats. Myocardial, plasma and extracardiac tissue amiodarone concentrations were analysed by high-performance liquid chromatography.

Results: The amiodarone-loaded hydrogel produced therapeutic drug concentrations in the right atrium up to 21 days after application. In this period, AERP and conduction time were prolonged, while RAR inducibility was reduced $(P<0.05)$ compared to animals treated with drug-free hydrogel. Mean amiodarone concentrations in the right atrium were 1 order of magnitude higher than in other heart chambers and 2 orders of magnitude higher than in extracardiac tissues. Plasma amiodarone levels remained below the detection limit $(<10 \mathrm{ng} / \mathrm{ml})$ during the 28 day follow-up.

Conclusions: Epicardial application of an amiodarone-releasing hydrogel reduces atrial vulnerability to tachyarrhythmias up to 3 weeks, while extracardiac drug levels remain low. Therefore, amiodarone-releasing hydrogel could be applied during cardiac surgery to prevent postoperative AF at minimal risk for extracardiac adverse side effects. 


\section{INTRODUCTION}

Amiodarone is currently the most effective drug for sinus rhythm maintenance. ${ }^{1-3}$ Originally categorized as a class III antiarrhythmic agent, amiodarone also displays class I, II, and IV effects, which differ between short-term and long-term therapy. ${ }^{4}$ In spite of its superior efficacy, amiodarone therapy has several disadvantages, including requirement of a loading period, interactions with many other drugs, and, most importantly, serious extracardiac side effects such as thyroid dysfunction, pulmonary toxicity and hepatic toxicity. 5,6 These are in part attributed to the relatively high drug concentrations required with systemic administration. Local drug delivery could minimize amiodarone's disadvantages, given that direct drug diffusion into the target tissue would eliminate the need for a loading period, while low systemic drug levels would reduce drug interactions and systemic adverse effects.

The concept of local amiodarone delivery to the myocardium has been investigated in several animal studies by short-term $(\leq 3 \mathrm{~h})$ intrapericardial infusion of amiodarone solutions. ${ }^{7-9}$ Although results are encouraging, most arrhythmias require a longer duration of treatment, but long-term catheter-based intrapericardial infusion may increase risks of infections and fibrosis. ${ }^{10,11}$ An interesting alternative for catheter-based approaches is the use of drug-releasing biomaterials. Previous studies demonstrated successful sustained drug delivery to the ventricular myocardium via intrapericardial infusion of microspheres or application of matrices to the ventricular epicardium. ${ }^{12,13}$ However, epicardial application of a drug-releasing matrix to the atrium in order to promote atrial selectivity has not been previously investigated.

The prevention of atrial tachyarrhythmias after cardiac surgery offers an ideal clinical entity to evaluate the concept of long-term epicardial drug delivery. A twocomponent surgical sealant (CoSeal ${ }^{\circledR}$, Angiotech Pharmaceuticals, Vancouver, Canada), based on biodegradable poly(ethylene glycol) (PEG) polymers that form a hydrogel, is already used for prevention of pericardial adhesions after cardiac surgery. ${ }^{14,15}$ We reasoned that addition of amiodarone to this hydrogel would produce a drug-releasing matrix, capable of sustained ( $>2$ weeks) suppression of atrial tachyarrhythmias, while systemic drug levels remain low. To test this hypothesis, we investigated drug distribution and electrophysiological effects after epicardial application of amiodarone-loaded hydrogels to the atria of goats.

\section{METHODS}

Animal handling was in accordance with the Guide for the Care and Use of Laboratory Animals published by the National Institutes of Health (NIH publication No. 85-23, 
revised 1996). In addition, this study protocol was approved by the Animal Studies Committee of the University of Maastricht.

Sixteen domestic goats, weighing 49 to $75 \mathrm{~kg}$, were randomised to 2 groups: drug-free hydrogel (DFH, n=6) and amiodarone-loaded hydrogel (ALH, n=10). All animals of the DFH group and 6 animals of the ALH group were chronically instrumented with epicardial electrodes, while 4 other animals of the ALH group were only used for pharmacokinetic studies.

\section{Hydrogel Preparation}

A sprayable polymeric matrix, CoSeal ${ }^{\circledR}$ Surgical Sealant (trademark of Angiotech Pharmaceuticals, Vancouver, Canada), was obtained via Baxter Healthcare (Fremont, CA, USA). The kit is composed of two synthetic PEGs, a dilute hydrogen chloride solution and a sodium phosphate/sodium carbonate solution. When mixed and sprayed over the epicardium, these components form a hydrogel that adheres to the epicardium. ${ }^{16}$

For goats of the DFH group, CoSeal was prepared according to the instructions for use. For goats of the ALH group, amiodarone hydrochloride powder (SigmaAldrich, St. Louis, MO, USA) was first added to the PEG powder, and the combined powder and solutions were mixed according to the CoSeal instructions for use, to produce a hydrogel with an amiodarone concentration of $25 \mathrm{mg} / \mathrm{ml}$.

Immediately after preparation, hydrogels were sprayed over the right atrial lateral wall. The volume of hydrogel applied in both groups corresponded to 0.04 $\mathrm{ml} / \mathrm{kg}$ bodyweight. Hence, goats of the ALH group received $1 \mathrm{mg}$ amiodarone per $\mathrm{kg}$ bodyweight. This dose was selected based on pilot experiments which demonstrated an increase in atrial effective refractory periods during three weeks after application of hydrogel.

\section{Surgical Procedure}

Anesthesia was induced by thiopental $20 \mathrm{mg} / \mathrm{kg}$ IV, and maintained with 2-3\% isoflurane in a 1:1 mixture of oxygen and air. Buprenorfine $(10 \mu \mathrm{g} / \mathrm{kg} \mathrm{IV})$ was used for analgesia. Throughout the procedure, limb-lead ECG, arterial blood pressure, endexpiratory $\mathrm{CO}_{2}$ and oxygen saturation (pulse oximeter) were monitored. A rectal temperature of 38 to $39{ }^{\circ} \mathrm{C}$ was maintained by an external heating pad. Fluid loss was compensated with saline ( $0.9 \%$ ) at 5 to $8 \mathrm{ml} / \mathrm{kg} / \mathrm{h}$ via a peripheral venous catheter.

Following a right intercostal thoracotomy, the pericardium above the right atrial lateral wall was opened. In the animals planned for electrophysiological studies, 3 silicone patches of $10 \times 10 \mathrm{~mm}$, each containing 4 silver unipolar electrodes $(1.5$ $\mathrm{mm}$ diameter, $5 \mathrm{~mm}$ interelectrode distance), were sutured in line to the right atrial 
lateral wall, between the cranial and caudal caval veins. Following baseline measurements, hydrogel (loaded with or without amiodarone) was sprayed over the right atrial lateral wall and measurements were repeated after $1 \mathrm{~h}$. Then the pericardium was approximated and sutured, and the thorax was closed, while the electrode leads were tunneled subcutaneously to the neck and exteriorized. Animals received twice-daily buprenorphine $(10 \mu \mathrm{g} / \mathrm{kg}$ IM) postoperatively for 3 days. Prophylactic ampicillin (20 mg/kg IM) was given before and directly after surgery.

\section{Electrophysiological Protocols}

Measurements were performed during the surgical procedure and 1, 2, 3, 4, 7, 9, 11, $14,16,18,21,23,25$ and 28 days after the surgical procedure. An electrophysiology monitoring system with an integrated amplifier/stimulator (EPTracer 38, CardioTek, Maastricht-Airport, The Netherlands) was used to simultaneously record limb-lead ECG and six bipolar electrograms from the 3 silicone patches sutured to the right atrial lateral wall. ECG parameters (RR, PQ, QT, and maximal $\mathrm{T}_{\text {peak }}-\mathrm{T}_{\text {end }}$ intervals, and $\mathrm{P}$ and QRS widths) were measured during normal sinus rhythm.

Atrial effective refractory periods (ERP) were measured during bipolar pacing at 4 times the threshold. After 10 basic stimuli (S1) with a 400-ms interval, an extrastimulus (S2) was applied. Starting from well within the refractory period, the S1-S2 interval was increased in steps of $2 \mathrm{~ms}$. The longest S1-S2 interval that failed to capture was defined as the ERP.

Atrial conduction times were recorded during 400-ms interval pacing at the silicone patch closest to the cranial caval vein. The time between stimulus artifact and the corresponding atrial deflection of the electrogram at the silicone patch closest to the caudal caval vein (i.e. the conduction delay across the crista terminalis) was defined as the conduction time.

Vulnerability to atrial tachyarrhythmias was evaluated by applying $50-\mathrm{Hz}$ burst pacing during $1 \mathrm{~s}$. A rapid atrial response (RAR) was considered inducible if a rapid irregular rhythm lasting $>1$ s occurred after the burst stimulus. For quantification of RAR inducibility, the percentages of RAR inductions out of 20 attempts were calculated. Duration of RAR episodes was measured and the medians were calculated.

\section{Pharmacokinetic Studies}

At days 1, 2, 3, 4, 7, 9, 11, 14, 16, 18, 21, 23, 25 and 28 after hydrogel application, blood samples were drawn from a peripheral vein of the hind limb via a venflon cannula and collected in EDTA-containing vacutainer tubes (Becton-Dickinson, Franklin Lakes, New Jersey, USA). After centrifugation, the plasma was stored at $-80{ }^{\circ} \mathrm{C}$. Six goats of the ALH group were euthanized with intravenous sodium pen- 
tobarbitone at $3 \mathrm{~h}$, and 1, 7, 14, 21 and 28 days after hydrogel application. Immediately after euthanasia, the heart was excised and the lateral right atrium and freewall sections of $4 \mathrm{~cm}^{2}$ from the left atrium and both ventricles were dissected. Samples were also taken from the pericardium overlying the right atrium, pericardial fluid, the medial part of the cranial lobe of the right lung, the skeletal muscle of the hind limb, liver and abdominal fat. Tissue samples were frozen in liquid nitrogen and stored at $-80{ }^{\circ} \mathrm{C}$. Myocardial samples were freeze-microtomed parallel to the epicardial surface in slices of $500 \mu \mathrm{m}$ for measurement of transmural drug concentration gradients. Amiodarone and desethylamiodarone concentrations in plasma, pericardial fluid and tissue samples were determined by high-performance liquid chromatography (HPLC) coupled with UV detection. ${ }^{17}$

\section{Statistical Analysis}

Data are presented as mean \pm SD. A Kruskal-Wallis test was used for multiple comparisons. Post-hoc tests included a Mann-Whitney $U$ test for calculating between group effects, a Wilcoxon signed rank test for calculating within-group effects, and a Bonferroni correction. Differences were defined statistically significant at a twotailed $P$ value of $<0.05$.

\section{RESULTS}

\section{Tolerability of Epicardial Application of Hydrogel}

All animals were in normal sinus rhythm at the beginning of the study and were hemodynamically stable during the surgical procedures. No complications occurred during follow-up (maximal 28 days) after hydrogel application in ALH and DFH groups. Application of amiodarone-loaded hydrogel did not produce significant changes in ECG rhythm and morphology compared to drug-free hydrogel (Table 1). However, a progressive increase in RR interval was observed in both groups during follow-up. This is probably a result of animal habituation, because similar ECG changes were seen in animals in which no hydrogel was applied (data not shown). At necropsy, neither pericardial adhesions nor signs of pericardial and myocardial damage were observed. 
Table 1. ECG parameters

\begin{tabular}{lcccccccc}
\hline & & \multicolumn{7}{c}{ Time after hydrogel application (days) } \\
\cline { 3 - 8 } & & 0 & 1 & 3 & 7 & 14 & 21 & 28 \\
\hline RR interval (ms) & ALH & $450 \pm 48$ & $492 \pm 52$ & $494 \pm 53$ & $507 \pm 40$ & $619 \pm 60$ & $635 \pm 54$ & $641 \pm 47$ \\
& DFH & $466 \pm 46$ & $480 \pm 43$ & $461 \pm 46$ & $477 \pm 48$ & $585 \pm 78$ & $628 \pm 52$ & $639 \pm 60$ \\
P width (ms) & ALH & $62 \pm 6$ & $68 \pm 5$ & $69 \pm 6$ & $71 \pm 4$ & $72 \pm 5$ & $72 \pm 5$ & $72 \pm 7$ \\
& DFH & $60 \pm 5$ & $62 \pm 5$ & $64 \pm 5$ & $67 \pm 6$ & $69 \pm 4$ & $71 \pm 5$ & $73 \pm 4$ \\
PQ interval (ms) & ALH & $110 \pm 13$ & $114 \pm 14$ & $128 \pm 16$ & $136 \pm 11$ & $136 \pm 11$ & $139 \pm 9$ & $139 \pm 9$ \\
& DFH & $108 \pm 11$ & $107 \pm 12$ & $118 \pm 13$ & $129 \pm 12$ & $133 \pm 9$ & $136 \pm 14$ & $136 \pm 11$ \\
QRS width (ms) & ALH & $63 \pm 4$ & $65 \pm 5$ & $65 \pm 4$ & $65 \pm 5$ & $64 \pm 6$ & $65 \pm 6$ & $65 \pm 5$ \\
& DFH & $62 \pm 5$ & $62 \pm 5$ & $62 \pm 4$ & $63 \pm 4$ & $63 \pm 5$ & $64 \pm 5$ & $64 \pm 5$ \\
QT interval (ms) & ALH & $271 \pm 20$ & $281 \pm 15$ & $282 \pm 23$ & $286 \pm 20$ & $314 \pm 22$ & $318 \pm 21$ & $319 \pm 22$ \\
& DFH & $269 \pm 17$ & $274 \pm 26$ & $267 \pm 19$ & $273 \pm 20$ & $301 \pm 21$ & $312 \pm 21$ & $315 \pm 28$ \\
& ALH & $35 \pm 6$ & $39 \pm 5$ & $37 \pm 8$ & $40 \pm 8$ & $40 \pm 7$ & $42 \pm 5$ & $44 \pm 6$ \\
& DFH & $37 \pm 6$ & $40 \pm 5$ & $37 \pm 5$ & $39 \pm 5$ & $42 \pm 6$ & $45 \pm 7$ & $46 \pm 7$ \\
\hline
\end{tabular}

Presented data were obtained from ECG recordings during normal sinus rhythm. Values are mean \pm SD. $\mathrm{n}=6$ for both amiodarone-loaded hydrogel (ALH) and drug-free hydrogel (DFH) groups. None of the differences between groups were statistically significant.

\section{Amiodarone and Desethylamiodarone Distribution}

Epicardial, endocardial, pericardial and peripheral tissue amiodarone concentrations at various times after hydrogel application are plotted in Figure 1. To study initial drug release from amiodarone-loaded hydrogel, one animal was euthanized 3 $\mathrm{h}$ after hydrogel application. HPLC-analysis revealed rapid distribution to the right atrium with very high tissue drug concentrations in the right atrial epicardium $(2226 \mu \mathrm{g} / \mathrm{g})$ and high concentrations in the right atrial endocardium $(228 \mu \mathrm{g} / \mathrm{g})$. Amiodarone levels in left atrium and both ventricles were low $(0.53$ to $4.00 \mu \mathrm{g} / \mathrm{g})$, while even lower levels were observed in extracardiac tissues (0.19 to $0.25 \mu \mathrm{g} / \mathrm{g}$ ).

After $24 \mathrm{~h}$ amiodarone concentrations were still highest in the right atrial epicardium, but high levels were also found in the epicardium of the left atrium and both ventricles, the endocardium of both atria, and in pericardial tissue. In the following 27 days cardiac amiodarone levels decreased seemingly mono-exponentially ( $\mathrm{t}_{1 / 2}$ approximately 3.5 days) at similar rates for all heart chambers. Mean amiodarone concentrations in the right atria from days 1 to 28 after hydrogel application were 3.4, 8.8 and 11.9 times higher than in the left atria, right ventricles and left ventricles, respectively. Regarding extracardiac tissues, peak amiodarone concentrations were low (1.14 to $2.68 \mu \mathrm{g} / \mathrm{g}$ at $24 \mathrm{~h}$ after hydrogel application) and decreased slower ( $\mathrm{t}_{1 / 2}$ approximately 7.5 days) than in cardiac tissues. 
Concentrations of desethylamiodarone (the primary active metabolite of amiodarone) in tissues peaked at $24 \mathrm{~h}$ after hydrogel application, ranging from $0.99 \mu \mathrm{g} / \mathrm{g}$ in abdominal fat to $7.29 \mu \mathrm{g} / \mathrm{g}$ in the epicardium of the right atrium, and decreased similarly to amiodarone concentrations in the following 27 days (data not shown). The higher cardiac than extracardiac desethylamiodarone levels suggest that a fraction of amiodarone is deethylated in the cardiac tissue itself.

Plasma concentrations of both amiodarone and desethylamiodarone remained below detection limits $(<10 \mathrm{ng} / \mathrm{ml})$ during 28 days of follow-up. Very low drug plasma levels are consistent with the low drug concentrations in extracardiac tissues and the extremely large volumes of distribution of both amiodarone and desethylamiodarone. ${ }^{18}$ Large volumes of distribution due to amiodarone's tissue affinity were also reflected by the high tissue/fluid drug concentration ratios of $>100$ observed for pericardial tissue and fluid (Figure 1C).

\section{Atrial Electrophysiology}

\section{Effective Refractory Period}

Before hydrogel application, ERPs measured at the right atrial epicardium did not significantly differ between ALH and DFH groups (Figure 2A). Drug-free hydrogels did not produce ERP changes, whereas application of amiodarone-loaded hydrogels produced a significant increase in right atrial ERP from $164 \pm 14 \mathrm{~ms}$ before hydrogel application to $191 \pm 14 \mathrm{~ms}$ at $24 \mathrm{~h}$ after hydrogel application. ERPs remained significantly higher in the ALH group than in the DFH group up to 18 days after hydrogel application.

\section{Conduction Time}

Figure $2 \mathrm{~B}$ shows changes in conduction times relative to baseline measurements (conduction times did not differ between ALH and DFH groups at baseline). Conduction times tended to shorten after application of drug-free hydrogels (mean changes ranging from -6.2 to $-10.1 \%$ ), but prolonged after application of amiodarone-loaded hydrogels (mean changes ranging from +2.1 to $+13.1 \%$ ). Changes in conduction times significantly differed between $\mathrm{ALH}$ and $\mathrm{DFH}$ groups from day 1 to day 25 after hydrogel applications. 
A

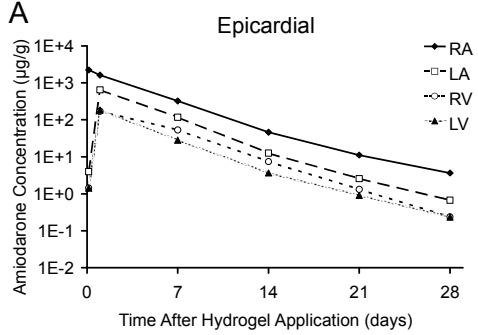

C

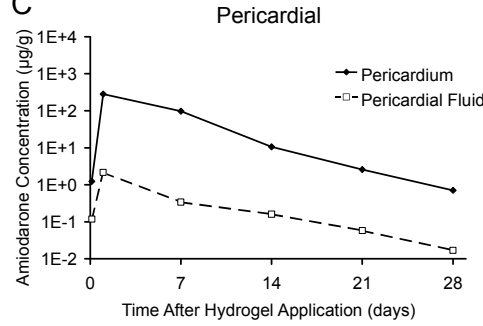

B

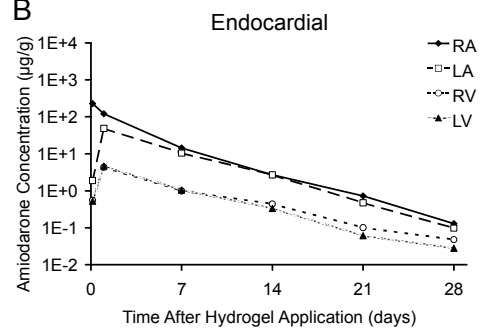

D

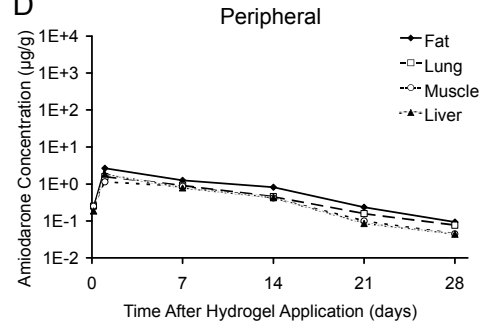

Figure 1. Amiodarone distribution. A, Epicardial amiodarone concentrations in right atrium (RA), left atrium (LA), right ventricle (RV) and left ventricle (LV). B, Endocardial amiodarone concentrations in RA, LA, RV and LV. C, Amiodarone concentrations in pericardium and pericardial fluid. D, Amiodarone concentrations in abdominal fat, the medial part of the cranial lobe of the right lung, skeletal muscle of the hind limb, and liver. Data were obtained 3 hours, and 1, 7, 14, 21 and 28 days after hydrogel application; $\mathrm{n}=1$ for each time point.

A

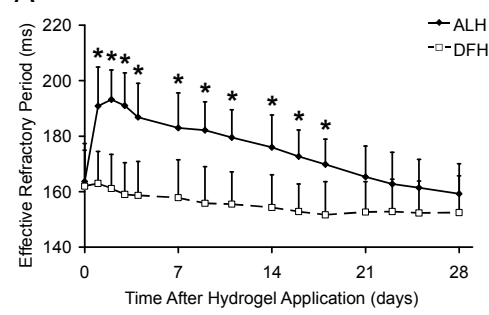

C

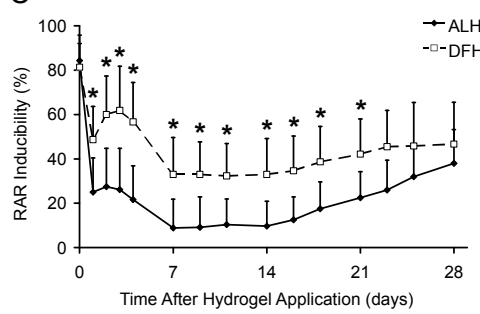

B

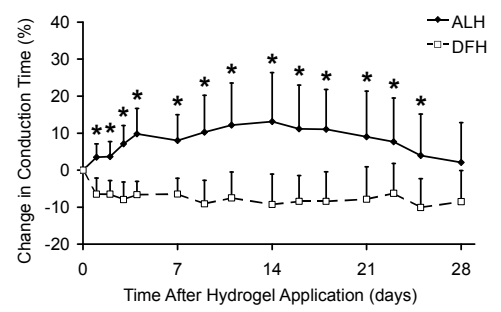

D

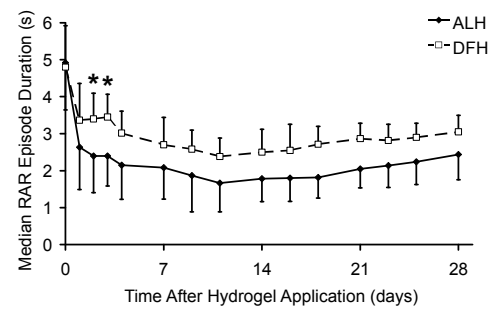

Figure 2. Atrial electrophysiology. A, Effective refractory period. B, Change in conduction time relative to baseline measurements. C, Rapid atrial response (RAR) inducibility. D, Median RAR duration. $n=6$ for both amiodarone-loaded hydrogel (ALH) and drug-free hydrogel (DFH) groups. ${ }^{*} P<0.05$. 
Response to Burst Pacing

Representative electrograms of rapid atrial responses to burst pacing at 14 days after hydrogel application are depicted in Figure 3. During baseline measurements, which were performed under anesthesia, burst pacing produced RARs in $84 \pm 11 \%$ and $81 \pm 11 \%$ of attempts in ALH and DFH groups, respectively (Figure 2C). RAR inducibility measured in awake animals during 28 days of follow-up showed a distinct pattern in both groups; following a decrease at $24 \mathrm{~h}$ after hydrogel application, RAR inducibility peaked at days 2 and 3, then remained relatively low and constant from days 7 to 14 , and finally increased from days 14 to 28. Absolute RAR inducibility was significantly lower in the ALH group than in the DFH group up to 21 days after hydrogel application. RAR episodes generally lasted several seconds and tended to be shorter in the ALH group, although statistical significance was only reached at days 2 and 3 after hydrogel application (Figure 2D).

Amiodarone-loaded hydrogel

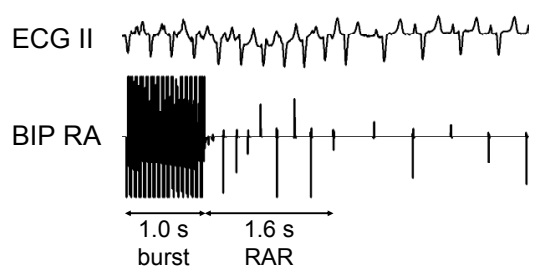

Drug-free hydrogel

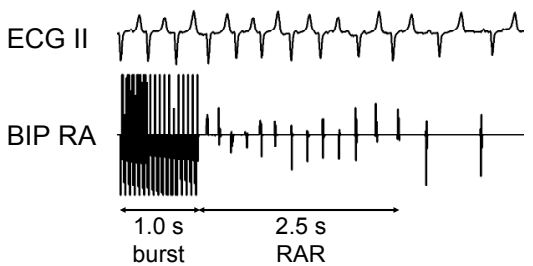

Figure 3. Typical responses to burst pacing 14 days after hydrogel application. BIP RA indicates right atrial bipolar electrode; RAR, rapid atrial response. 


\section{DISCUSSION}

The present study demonstrates that epicardial application of an amiodaroneloaded hydrogel produces therapeutic atrial drug concentrations for a prolonged period, while systemic drug concentrations remain low. In ALH-treated goats, atrial ERP and conduction time increased, and atrial vulnerability to burst-pacing induced tachyarrhythmias decreased.

Previous studies in dogs and pigs showed that short-term $(\leq 3 \mathrm{~h})$ local amiodarone delivery to the myocardium can be achieved via intrapericardial infusion of amiodarone solutions. ${ }^{78}$ High amiodarone concentrations were observed in atria, sinoatrial node and ventricular subepicardium, and lower concentrations in interventricular septum and ventricular subendocardium. Plasma amiodarone levels were in the range of $10-100 \mathrm{ng} / \mathrm{ml}$, i.e. 1-2 orders of magnitude lower than levels normally present during intravenous or chronic oral amiodarone therapy. In a recent study in our laboratory, we showed that transmural drug concentration gradients during intrapericardial delivery of amiodarone were not only present in ventricles, but also in atria. ${ }^{9}$ Although epicardial and endocardial atrial ERPs were both prolonged, the absolute difference between them (transmural dispersion of refractoriness) did not increase. The same held true for epicardial and endocardial conduction times. Intrapericardially delivered amiodarone significantly reduced epicardial and endocardial inducibility of atrial tachyarrhythmias compared to an equal intravenous dose of amiodarone.

In the present report we show drug distribution and electrophysiological effects during sustained local amiodarone delivery. The high amiodarone concentrations in the right atrium already after $3 \mathrm{~h}$ of hydrogel application point to an initial burst release phase. Drug levels decreased mono-exponentially over the next 4 weeks. Since the hydrogel degrades within 4 weeks, the latter phase is likely a combination of diffusion- and degradation-controlled drug release. Although amiodarone distribution to other heart chambers via diffusion into the pericardial fluid, subepicardial lymphatics and blood vessels was expected, highest drug concentrations were still observed in the right atrium. Therefore, application of a tissue-adherent drugreleasing hydrogel allows for more localized drug delivery than intrapericardial infusion of drug solutions. However, endocardial amiodarone concentrations in the right atrium were lower than epicardial concentrations in both atria and ventricles. This can be a result of slower transmural (transversal) than epicardial (longitudinal) drug distribution, and/or lower epicardial than endocardial drug clearance. Given the fact that the epicardial drug distribution may be facilitated by drug uptake and subsequent release via the pericardial fluid, a relatively rapid longitudinal versus transversal distribution seems likely. Transmural drug concentration gradients persisted in all heart chambers. Despite the long treatment duration compared to 
studies that used intrapericardial amiodarone delivery, extracardiac tissue drug levels remained low, while plasma drug levels never surpassed the detection limit $(10 \mathrm{ng} / \mathrm{ml})$. This indicates slow diffusion to plasma and relatively rapid redistribution from plasma to extracardiac tissues, as can be expected of a drug with a large volume of distribution due to high tissue affinity. ${ }^{18}$

In animals treated with drug-free hydrogel, atrial vulnerability to burst-pacing induced tachyarrhythmias showed a peak on days 2 and 3. Although sustained episodes of atrial fibrillation were not observed, this peak coincides with the highest incidence of atrial fibrillation following cardiac surgery observed in patients on postoperative days 2 and $3 .{ }^{19}$ Application of amiodarone-loaded hydrogel significantly reduced peak occurrence in the present study, which suggest that this method may also be efficacious in preventing postoperative atrial fibrillation. The potent antiarrhythmic effect in the first days following hydrogel application can be mainly attributed to amiodarone's class III action, given the substantial prolongation of atrial ERP. Atrial amiodarone tissue concentrations and effects on ERP showed a good correlation, whereas a less consistent relationship was observed for changes in conduction time. Electrophysiological effects of amiodarone are not only dependent on tissue drug concentrations, but also on treatment duration, which may explain these findings. ${ }^{4}$

Previous methods of sustained local drug delivery to the myocardium relied on chronic intrapericardial catheterization or the use of non-biodegradable polymeric drug delivery systems. ${ }^{10,11,20-23}$ However, these methods may increase risks of infections and fibrosis. Furthermore in non-biodegradable systems such complications may necessitate surgical removal of the drug delivery matrix. In one study biodegradable poly(lactic-co-glycolic acid) (PLGA) microspheres loaded with sotalol were injected intrapericardially, producing sustained (30 days) intrapericardial drug delivery. ${ }^{12}$ The disadvantage of this system is that the drug-loaded microspheres are highly mobile and do not provide further localised delivery when compared to an adhesive hydrogel. The PEG-based hydrogel used in the present study displays both biodegradability and immediate tissue-adherence during in situ forming, allowing more localized drug delivery. Moreover, clinical safety of epicardial application of this hydrogel has already been demonstrated. ${ }^{14,15}$ Amiodarone loading did not affect hydrogel tolerability in the goats currently studied. QRS width, QT interval and maximal $\mathrm{T}_{\text {peak }}-\mathrm{T}_{\text {end }}$ interval remained similar in both experimental groups, indicating that local amiodarone delivery had minimal effects on global ventricular electrophysiology. This may be explained by low ventricular midmyocardial and endocardial amiodarone concentrations in combination with the intrinsic atrial predominant electrophysiological effects of amiodarone. ${ }^{24}$

In addition to prevention of postoperative $\mathrm{AF}$, other potential clinical applications of amiodarone-releasing hydrogels include treatment of paroxysmal atrial 
fibrillation and prevention of epicardial focus-induced ventricular arrhythmias. Development of hydrogels capable of even longer periods of sustained drug release, and minimally-invasive percutaneous techniques for hydrogel delivery may promote clinical applicability. Regarding the latter, promising approaches, such as subxiphoid and transatrial access, have already been evaluated. ${ }^{25,26}$ In patients with paroxysmal atrial fibrillation the pulmonary veins are important in arrhythmogenesis. ${ }^{27}$ It would be interesting to study effects of amiodarone-releasing hydrogel application around the ostia of the pulmonary veins. While the present study focused on local delivery of drugs, epicardially applied matrices can also be used for delivery of proteins, gene vectors and cells. ${ }^{13,28-31}$ Particularly PEG-based hydrogels are highly biocompatible and offer great flexibility in tailoring structural and functional matrix characteristics. ${ }^{31}$ Therefore, these polymeric delivery systems may facilitate a broad spectrum of cardiac therapeutic applications, including treatment of arrhythmias, ischemic heart disease and heart failure.

\section{Limitations}

The canine sterile pericarditis model is the best known animal model to study postoperative atrial flutter and AF. ${ }^{32,33}$ The hydrogel used in the present study reduces epicardial inflammation, which explains its efficacy in preventing pericardial adhesions after cardiac surgery. ${ }^{14,15}$ Because epicardial inflammation is the causal factor of AF in the sterile pericarditis model, hydrogel application would make this model ineffective. Furthermore, epicardial and pericardial inflammation in regions not covered by hydrogel would produce fibrosis, thereby altering transmural amiodarone diffusion. Therefore, the canine sterile pericarditis model was not used in the present study. In chronic AF models, such as pacing-induced atrial tachycardia, electrophysiological mechanisms differ from underlying mechanisms in postoperative AF, which may reduce efficacy of local drug delivery. ${ }^{34,35}$ Sustained episodes of atrial fibrillation did not occur in the present study with healthy goats, but RAR inducibility followed a pattern similar to incidence of clinical postoperative AF showing a peak on days 2 and 3. Although amiodarone-loaded hydrogel significantly increased conduction time, as well as the atrial effective refractory period, effects on RAR inducibility were less prominent. However, this is an inherent limitation of the experimental model.

Only one dose of amiodarone hydrochloride powder and one type of hydrogel was used to prepare drug-loaded hydrogels in this study. Other hydrogel formulations, by changing polymer chemistry or drug dose, or using dissolved amiodarone, may feature different drug release characteristics.

Histologic studies of the pericardium, atria and ventricles were not performed in the present study. Although no macroscopic adverse effects were observed, high 
local amiodarone concentrations might produce toxic effects, such as necrosis or inflammation. Thus, further research is needed to determine the safety of this method.

\section{Conclusions}

Epicardial application of anamiodarone-releasing adhesive hydrogel is well tolerated and allows antiarrhythmic efficacy at very low systemic drug levels, which minimizes the risk for extracardiac adverse effects. In addition, a loading period is not required. Amiodarone-releasing hydrogel could be applied during cardiac surgery to prevent postoperative AF. 


\section{REFERENCES}

1. Zimetbaum P. Amiodarone for atrial fibrillation. N Engl J Med 2007;356:935-41.

2. Lafuente-Lafuente C, Mouly S, Longas-Tejero MA, Bergmann JF. Antiarrhythmics for maintaining sinus rhythm after cardioversion of atrial fibrillation. Cochrane Database Syst Rev 2007:CD005049.

3. Singh BN. Amiodarone as paradigm for developing new drugs for atrial fibrillation. J Cardiovasc Pharmacol 2008;52:300-5.

4. Kodama I, Kamiya K, Toyama J. Amiodarone: ionic and cellular mechanisms of action of the most promising class III agent. Am J Cardiol 1999;84:20R-8R.

5. Vassallo P, Trohman RG. Prescribing amiodarone: an evidence-based review of clinical indications. JAMA 2007;298:1312-22.

6. Camm AJ. Safety considerations in the pharmacological management of atrial fibrillation. Int $J$ Cardiol 2008;127:299-306.

7. Ayers GM, Rho TH, Ben-David J, Besch HR, Zipes DP. Amiodarone instilled into the canine pericardial sac migrates transmurally to produce electrophysiologic effects and suppress atrial fibrillation. J Cardiovasc Electrophysiol 1996;7:713-21.

8. Darsinos JT, Karli JN, Samouilidou EC, Krumbholz B, Pistevos AC, Levis GM. Distribution of amiodarone in heart tissues following intrapericardial administration. Int J Clin Pharmacol Ther 1999;37:301-6.

9. Bolderman RW, Hermans JJ, Rademakers LM, et al. Intrapericardial delivery of amiodarone and sotalol: atrial transmural drug distribution and electrophysiological effects. J Cardiovasc Pharmacol 2009;54:355-63.

10. Kolettis TM, Kazakos N, Katsouras CS, et al. Intrapericardial drug delivery: pharmacologic properties and long-term safety in swine. Int J Cardiol 2005;99: 415-21.

11. Bartoli CR, Akiyama I, Godleski JJ, Verrier RL. Long-term pericardial catheterization is associated with minimum foreign-body response. Catheter Cardiovasc Interv 2007;70:221-7.

12. Labhasetwar V, Underwood T, Gallagher M, Murphy G, Langberg J, Levy RJ. Sotalol controlled-release systems for arrhythmias: in vitro characterization, in vivo drug disposition, and electrophysiologic effects. J Pharm Sci 1994;83:156-64.

13. Zhang G, Nakamura $Y$, Wang $X, H u$ Q, Suggs LJ, Zhang J. Controlled release of stromal cellderived factor-1 alpha in situ increases c-kit+cell homing to the infarcted heart. Tissue Eng 2007;13:2063-71.

14. Hendrikx M, Mees U, Hill AC, Egbert B, Coker GT, Estridge TD. Evaluation of a novel synthetic sealant for inhibition of cardiac adhesions and clinical experience in cardiac surgery procedures. Heart Surg Forum 2001;4:204-10.

15. Konertz WF, Kostelka M, Mohr FW, et al. Reducing the incidence and severity of pericardial adhesions with a sprayable polymeric matrix. Ann Thorac Surg 2003;76:1270-4.

16. Wallace DG, Cruise GM, Rhee WM, et al. A tissue sealant based on reactive multifunctional polyethylene glycol. J Biomed Mater Res 2001;58:545-55.

17. Bolderman RW, Hermans JJ, Maessen JG. Determination of the class III antiarrhythmic drugs dronedarone and amiodarone, and their principal metabolites in plasma and myocardium by high-performance liquid chromatography and UV-detection. J Chromatogr B Analyt Technol Biomed Life Sci 2009;877:1727-31.

18. Holt DW, Tucker GT, Jackson PR, Storey GC. Amiodarone pharmacokinetics. Am Heart J 1983;106:840-7. 
19. Mathew JP, Fontes ML, Tudor IC, et al. A multicenter risk index for atrial fibrillation after cardiac surgery. JAMA 2004;291:1720-9.

20. Sintov A, Scott W, Dick M, Levy RJ. Cardiac controlled release for arrhythmia therapy: lidocaine-polyurethane matrix studies. J Control Release 1988;8:157-65.

21. Siden R, Flowers WE, Levy RJ. Epicardial propranolol administration for ventricular arrhythmias in dogs:matrix formulation and characterization. Biomaterials 1992;13:764-70.

22. Siden R, Kadish A, Flowers W, et al. Epicardial controlled-release verapamil prevents ventricular tachycardia episodes induced by acute ischemia in a canine model. J Cardiovasc Pharmacol 1992;19:798-809.

23. Labhasetwar V, Underwood T, Heil Jr RW, Gallagher M, Langberg J, Levy RJ. Epicardial administration of ibutilide from polyurethane matrices: effects on defibrillation threshold and electrophysiologic parameters. J Cardiovasc Pharmacol 1994;24:826-40.

24. Burashnikov A, Di Diego JM, Sicouri S, Ferreiro M, Carlsson L, Antzelevitch C. Atrialselective effects of chronic amiodarone in the management of atrial fibrillation. Heart Rhythm 2008;5:1735-42.

25. Verrier RL, Waxman S, Lovett EG, Moreno R. Transatrial access to the normal pericardial space: a novel approach for diagnostic sampling, pericardiocentesis, and therapeutic interventions. Circulation 1998;98:2331-3.

26. Maisch B, Ristic AD, Rupp H, Spodick DH. Pericardial access using the PerDUCER and flexible percutaneous pericardioscopy. Am J Cardiol 2001;88:1323-6.

27. Haissaguerre $\mathrm{M}$, Jais $\mathrm{P}$, Shah $\mathrm{DC}$, et al. Spontaneous initiation of atrial fibrillation by ectopic beats originating in the pulmonary veins. N Engl J Med 1998;339:659-66.

28. Kikuchi K, McDonald AD, Sasano T, Donahue JK. Targeted modification of atrial electrophysiology by homogeneous transmural atrial gene transfer. Circulation 2005;111:264-70.

29. Mei L, Jin X, Song C,WangM, Levy RJ. Immobilization of gene vectors on polyurethane surfaces using amonoclonal antibody for localized gene delivery. J GeneMed 2006;8:690-8.

30. Kutschka I, Chen IY, Kofidis T, et al. Collagen matrices enhance survival of transplanted cardiomyoblasts and contribute to functional improvement of ischemic rat hearts. Circulation 2006;114:I167-73.

31. Lin CC, Anseth KS. PEG hydrogels for the controlled release of biomolecules in regenerative medicine. Pharm Res 2009;26:631-43.

32. Page PL, Plumb VJ, Okumura K, Waldo AL. A new animal model of atrial flutter. J Am Coll Cardiol 1986;8:872-9.

33. Kumagai K, Khrestian C, Waldo AL. Simultaneous multisite mapping studies during induced atrial fibrillation in the sterile pericarditis model. Insights into the mechanism of its maintenance. Circulation 1997;95:511-21.

34. Vereckei A, Gorski JC, Ujhelyi M, Mehra R, Zipes DP. Intrapericardial ibutilide administration fails to terminate pacing-induced sustained atrial fibrillation in dogs. Cardiovasc Drugs Ther 2004;18:269-77.

35. Van Brakel TJ, Hermans JJ, Accord RE, et al. Effects of intrapericardial sotalol and flecainide on transmural atrial electrophysiology and atrial fibrillation. J Cardiovasc Electrophysiol 2009;20:207-15. 


\section{CHAPTER 5}

\section{Atrium-targeted drug delivery through an amiodarone-eluting bilayered patch}




\section{Abstract}

Objective: Clinical studies have demonstrated the efficacy of oral and intravenous amiodarone therapy to prevent postoperative atrial fibrillation. However, because of significant extracardiac side effects, only high-risk patients are eligible for prophylactic amiodarone therapy. This study addressed the hypothesis that atriumspecific drug delivery through an amiodarone-eluting epicardial patch reduces vulnerability to atrial tachyarrhythmias, whereas ventricular and plasma drug concentrations are minimized.

Methods: Right atrial epicardiums of goats were fitted with electrodes and a bilayered patch (poly[ethylene glycol]-based matrix and poly[lactide-co-caprolactone] backing layer) loaded with amiodarone (10 mg per patch, $\mathrm{n}=10$ ) or without drug $(n=6)$. Electrophysiologic parameters (atrial effective refractory period, conduction time, and rapid atrial response to burst pacing) and amiodarone levels in plasma and tissue were measured during 1 month's follow-up.

Results: Epicardial application of amiodarone-eluting patches produced persistently higher drug concentrations in the right atrium than in the left atrium, ventricles, and extracardiac tissues by 2 to 4 orders of magnitude. Atrial effective refractory period and conduction time increased, whereas rapid atrial response inducibility decreased significantly $(P<0.05)$ during the 1 -month follow-up compared with that seen in animals treated with drug free patches. Amiodarone concentrations in plasma remained undetectably low ( $<10 \mathrm{ng} / \mathrm{ml})$.

Conclusions: Atrium-specific drug delivery through an amiodarone-eluting patch produces therapeutic atrial drug concentrations, whereas ventricular and systemic drug levels are minimized. This study demonstrates that sustained targeted drug delivery to a specific heart chamber is feasible and might reduce the risk for ventricular and extracardiac adverse effects. Epicardial application of amiodarone eluting patches is a promising strategy to prevent postoperative atrial fibrillation. 


\section{INTRODUCTION}

Postoperative atrial fibrillation (POAF) is the most frequent complication of cardiac surgery, with incidence rates varying between $20 \%$ and $60 \%$ depending on the type of procedure and the criteria for diagnosis. ${ }^{1}$ POAF is associated with an increased risk of morbidity and mortality caused by stroke, heart failure, ventricular arrhythmias, thromboembolism, and bleeding from anticoagulation. ${ }^{2}$ Furthermore, POAF increases length of hospital stay, hospital readmission rate, and hospital costs.

According to the American College of Cardiology/American Heart Association/European Society of Cardiology 2006 joint guidelines, POAF should be prevented by treatment with an oral $\beta$-blocker or, in patients are at high risk, preoperative administration of amiodarone. ${ }^{3}$ Although amiodarone is probably the most effective drug, it is reserved as a second-line agent because of its associated risk of potentially severe extracardiac adverse effects, such as thyroid dysfunction, pulmonary toxicity, and hepatic toxicity. ${ }^{4}$ These effects are in part attributable to the relatively high drug concentrations required with systemic administration. Another disadvantage of amiodarone is the need for a loading period. ${ }^{5}$

Local drug delivery could minimize the ventricular and extracardiac adverse effects of amiodarone and could make a loading period superfluous. Several studies investigated intrapericardial infusion of amiodarone solutions to achieve local drug delivery. ${ }^{6-8}$ However, this method does not allow for atrium-specific drug targeting because the drug equally diffuses to the ventricular epicardium. Epicardial application of drug-releasing biomaterials might offer the advantage of a more localized (heart chamber-targeted) drug delivery, although diffusion to other heart chambers, partly through leakage to the pericardial fluid, is still possible. ${ }^{9}$

We reasoned that atrium-specific amiodarone therapy might be achieved if a bilayered patch would be applied epicardially in such a way that an amiodaroneloaded matrix faces the epicardium and an impermeable backing layer faces the pericardium. This study addressed the hypothesis that amiodarone-eluting bilayered patches reduce vulnerability to atrial tachyarrhythmias in chronically instrumented goats while ventricular and systemic drug concentrations are minimized. 


\section{METHODS}

All procedures were approved by the Animal Studies Committee of the University of Maastricht and performed according to the principles of "The guide for the care and use of laboratory animals" published by the National Institutes of Health (National Institutes of Health publication no. 85-23, revised 1996).

Domestic goats ( $\mathrm{n}=16$; weight, 47-67 $\mathrm{kg}$ ) were randomly assigned to a drug-free patch group (DFP group, $n=6$ ) or an amiodarone-loaded patch group (ALP group, $\mathrm{n}=10$ ). Four animals of the ALP group were only used for pharmacokinetic studies, whereas all other animals were chronically instrumented with epicardial electrodes.

\section{Patch Synthesis}

Preparation of Poly(ethylene glycol) 10,000 tetra(4-pentenoate)

Under a nitrogen atmosphere, $20 \mathrm{~g}$ of poly(ethylene glycol) (PEG) 10,000 4-arm hydroxyl (Nektar Therapeutics, San Carlos, Calif) was dissolved in $200 \mathrm{ml}$ of toluene and $50 \mathrm{ml}$ of dichloromethane and cooled with an ice bath. Triethylamine $(2.8$ $\mathrm{ml}$ ) was added to the clear solution. Then $2.4 \mathrm{ml}$ of 4 -pentenoylchloride (SigmaAldrich, St Louis, Mo) was added dropwise to the ice-cooled solution. The reaction was continued overnight at room temperature. Triethylamine salts were then filtered off in vacuo, and the clear filtrate was precipitated into 21 of ice-cold diethylether. The solid precipitate was collected and redissolved in $100 \mathrm{ml}$ of chloroform. This solution was reprecipitated into excess of hexane. The precipitate was collected and dried in vacuo. This yielded approximately $15 \mathrm{~g}$ (97.8\%) of starshaped PEG 10,000 tetra(4-pentenoate) as a white powder. The polymer was characterized by using ${ }^{1} \mathrm{H}$-nuclear magnetic resonance spectroscopy (at $300 \mathrm{MHz}, 22{ }^{\circ} \mathrm{C}$ with deuterated chloroform as a solvent and tetramethylsilane as an internal standard): $\delta(\mathrm{ppm})=5.79\left(\mathrm{~m}, 4 \mathrm{H},-\mathrm{CH}=\mathrm{CH}_{2}\right) ; 5.05\left(\mathrm{~m}, 8 \mathrm{H},-\mathrm{CH}=\mathrm{CH}_{2}\right) ; 4.22(\mathrm{t}, 8 \mathrm{H},-$ $\left.\mathrm{OCH}_{2} \mathrm{CH}_{2} \mathrm{O}(\mathrm{C}=\mathrm{O})-\right)$; 3.89-3.35 (m, 938H, $-\mathrm{OCH}_{2} \mathrm{CH}_{2} \mathrm{O}(\mathrm{C}=\mathrm{O})-$ and $\left.-\left(\mathrm{OCH}_{2} \mathrm{CH}_{2}\right)_{\mathrm{n}} \mathrm{O}-\right)$ and $\left.\mathrm{C}\left(\mathrm{CH}_{2} \mathrm{O}\right)_{4-}^{-}\right)$.

\section{Fabrication of Bilayered Patches}

Two grams of PEG 10,000 tetra(4-pentenoate) and $0.1 \mathrm{~g}$ adipic thioic acid (1,6hexane dithioic acid) were dissolved in $2 \mathrm{ml}$ of tetrahydrofuran. ${ }^{10}$ Photoinitiator Darocur 1173 (0.05 g; Ciba, Basel, Switzerland) and 10\% wt/wt (relative to PEG plus cross-linker) amiodarone powder (Sigma-Aldrich) were added to this solution. The suspension was stirred vigorously and transferred into circular Teflon molds (14$\mathrm{mm}$ diameter) on top of a $50-\mu \mathrm{m}$-thick elastic film composed of a poly(lactide-cocaprolactone) (PLCL) copolymer. The suspensions were exposed to UV light (5x1 $\mathrm{J} / \mathrm{cm}^{2}$ ) and dried in vacuo for several days, resulting in the formation of biodegrad- 
able bilayered discoidal patches composed of a amiodarone-loaded PEG matrix and a PLCL backing layer. Patches were $300 \mu \mathrm{m}$ thick, had a diameter of $13 \mathrm{~mm}$, weighed $101 \pm 4 \mathrm{mg}$, and contained $9.9 \pm 0.4 \mathrm{mg}$ of amiodarone. The amiodarone dose was selected based on pilot experiments, which demonstrated an increase in atrial effective refractory periods (ERPs) 28 days after patch application. Drug-free patches were synthesized according to the same procedure without the addition of amiodarone.

\section{Patch Application}

Goats were anesthetized with isoflurane (2\% to $3 \%$ ) with a 1:1 mixture of oxygen and air after induction of anesthesia with thiopental, $20 \mathrm{mg} / \mathrm{kg}$ administered intravenously. During surgical intervention, limb-lead electrocardiography (ECG), arterial blood pressure, temperature, end-expiratory $\mathrm{CO}_{2}$, and oxygen saturation (pulse oximetry) were monitored. Saline $(0.9 \%, 5-8 \mathrm{ml} / \mathrm{kg}$ per hour) was infused through a peripheral venous catheter. Buprenorphine $(10 \mu \mathrm{g} / \mathrm{kg}$ administered intravenously) was used as an analgesic.

A right intercostal thoracotomy was performed, and the right atrial lateral wall was exposed. In goats scheduled for chronic electrophysiologic studies, 2 silicone strips of $10 \times 10 \mathrm{~mm}$, each containing 4 silver unipolar electrodes (1.5-mm diameter and 5-mm interelectrode distance), were sutured to the right atrial lateral wall, one close to the superior caval vein and the other close to the inferior caval vein. After baseline measurements, a patch (loaded with or without amiodarone) was sutured to the right atrial lateral wall (between the 2 electrode-containing silicone strips), and electrophysiologic measurements were repeated after 1 hour. The pericardium and thorax were subsequently closed, and the electrode leads were tunneled subcutaneously to the neck and exteriorized. Animals were injected with prophylactic ampicillin (20 mg/kg administered intramuscularly) before and directly after surgical intervention. Buprenorphine $(10 \mu \mathrm{g} / \mathrm{kg}$ administered intramuscularly twice daily) was administered postoperatively for 3 days.

\section{Electrophysiologic Studies}

Electrophysiologic parameters were measured during surgical intervention and at days $1,2,3,4,7,9,11,14,16,18,21,23,25$, and 28 after surgical intervention. ECG limb leads and electrodes were connected to an electrophysiologic monitoring system with an integrated amplifier/stimulator (EP-Tracer 38; CardioTek, MaastrichtAirport, The Netherlands). From the ECG results obtained during sinus rhythm, R$\mathrm{R}, \mathrm{PQ}, \mathrm{QT}$, and maximal $\mathrm{T}_{\text {peak }}-\mathrm{T}_{\text {end }}$ intervals and P and QRS widths were measured. 
Four bipolar electrograms were recorded simultaneously from the 2 silicone strips flanking the patch.

For measurement of atrial ERP, 10 basic stimuli (S1, 400-ms interval) were applied by means of bipolar pacing at 4 times the threshold. After S1, an extra stimulus (S2) was applied starting from well within the refractory period. The S1-S2 interval was increased with 2-ms steps, and atrial ERP was defined as the longest S1S2 interval that failed to capture.

For measurement of conduction times, the atrium was paced (400-ms interval) at the silicone strip close to the superior caval vein. Conduction time was defined as the time between stimulus artifact and corresponding atrial deflection of the electrogram recorded at the silicone patch close to the inferior caval vein, representing the conduction delay across the crista terminalis.

Inducibility of a rapid atrial response (RAR) was measured by applying $50-\mathrm{Hz}$ burst pacing during 1 second. If the burst stimulus produced a rapid irregular rhythm lasting more than 1 second, RAR was considered inducible. RAR inducibility was quantified by the percentage of RAR inductions after 20 attempts, and median RAR episode durations were calculated.

\section{Drug Distribution Analysis}

Blood samples were regularly drawn from a peripheral vein of the hind limb through a Venflon cannula (Becton Dickinson, Franklin Lakes, NJ) up to 28 days after patch application. The blood was collected in ethylenediamine tetraacetic acidcontaining Vacutainer tubes (Becton Dickinson) and centrifuged to obtain plasma that was stored at $-80{ }^{\circ} \mathrm{C}$. At 3 hours and 1, 7, 14, 21, and 28 days after patch application, goats of the ALP group ( $\mathrm{n}=1$ for each time point) were killed with intravenous sodium pentobarbital, and the heart was excised. The lateral right atrium (RA) and free-wall sections $\left(4 \mathrm{~cm}^{2}\right)$ of the left atrium (LA) and both ventricles were dissected. In addition, samples of pericardium overlying the RA, pericardial fluid, the medial part of the cranial lobe of the right lung, skeletal muscle of the hind limb, and liver and abdominal fat were obtained. After immersion in liquid nitrogen for rapid freezing, tissue samples were stored at $-80{ }^{\circ} \mathrm{C}$. For measurement of transmural drug concentration gradients, myocardial samples were freeze-microtomed parallel to the epicardial surface in slices of $500 \mu \mathrm{m}$. High-performance liquid chromatography coupled with UV detection was used to determine amiodarone and desethylamiodarone concentrations in plasma, pericardial fluid, and tissue samples. ${ }^{11}$ 


\section{Statistics}

Data are expressed as means \pm standard deviations. Differences between means were calculated with a Kruskal-Wallis test for multiple comparisons. To determine the significance of differences of individual comparisons, Mann-Whitney U and Wilcoxon signed rank tests were used for between-group and within-group comparisons, respectively, with post hoc Bonferroni correction.

\section{RESUlts}

\section{Feasibility and Tolerability of Patch Application}

After baseline measurements, suturing of patches to the atria was achieved within 5 minutes in each animal, without significant bleeding or changes in blood pressure. Hemodynamic and ECG parameters remained stable during the remainder of the surgical procedures. No signs of discomfort, adverse effects, or complications were observed up to 28 days after patch application. Except for a 70-ms longer R-R interval in the ALP group than in the DFP group at day 3 after patch application, ECG parameters did not significantly differ between groups over the time period evaluated in the present study (Table 1). Gradual prolongations of R-R intervals observed in both groups during follow-up can be explained by animal habituation because similar ECG changes were observed in animals in which no patch was applied (data not shown). In all animals killed after 7 or more days, electrode strips and patches were covered by a thin $(<1 \mathrm{~mm})$ fibrous layer, without signs of pericarditis or myocarditis.

\section{Pharmacokinetic Feasibility of Atrium-Targeted Drug Delivery}

Epicardial application of amiodarone-eluting patches produced persistently higher drug concentrations in the RA than in the LA, right ventricle (RV), and left ventricle (LV; Figure 1). RA/LA, RA/RV, and RA/LV mean amiodarone concentration ratios at 3 hours after patch application were 40,67, and 76, respectively. These ratios increased to 1145, 2247, and 2167, respectively, at 28 days after patch application. Amiodarone concentrations reached peaks after 24 hours of patch application in all tissues studied. Cardiac tissue concentrations decreased in the next 27 days, with overall half-lives of 4 to 5 days (assuming monoexponential decrease). Peak amiodarone concentrations in extracardiac tissues were 3 to 4 orders of magnitude lower than right atrial peak drug concentrations and decreased to less than $10 \mathrm{ng} / \mathrm{ml}$ (detection limit) at 21 days after patch application. For all blood samples drawn during the 28-day follow-up period, plasma amiodarone levels were less than the detection 
limit. Because amiodarone has a high tissue affinity, lower drug levels in plasma than in tissues are expected. These findings are also in line with the lower drug levels measured in pericardial fluid than in pericardial tissue (Figure 1, C).

The primary active metabolite of amiodarone is desethylamiodarone. Desethylamiodarone levels in the RA were 3 orders of magnitude lower than amiodarone levels, whereas metabolite concentrations in the other heart chambers were 1 order of magnitude lower than parent drug concentrations (data not shown). Distribution of desethylamiodarone in extracardiac tissues was similar to amiodarone distribution. Plasma metabolite levels remained undetectably low during the 28-day followup period.

Table 1. ECG parameters

\begin{tabular}{lcccccccc}
\hline & & \multicolumn{7}{c}{ Time after patch application (days) } \\
\cline { 3 - 8 } & & 0 & 1 & 3 & 7 & 14 & 21 & 28 \\
\hline RR interval (ms) & ALP & $463 \pm 46$ & $473 \pm 44$ & $509 \pm 47^{*}$ & $546 \pm 51$ & $616 \pm 84$ & $656 \pm 82$ & $672 \pm 83$ \\
& DFP & $435 \pm 55$ & $452 \pm 51$ & $439 \pm 47$ & $488 \pm 58$ & $565 \pm 56$ & $626 \pm 66$ & $648 \pm 70$ \\
P width (ms) & ALP & $64 \pm 7$ & $68 \pm 6$ & $68 \pm 7$ & $71 \pm 6$ & $73 \pm 5$ & $74 \pm 5$ & $76 \pm 5$ \\
& DFP & $62 \pm 6$ & $62 \pm 7$ & $64 \pm 5$ & $66 \pm 7$ & $69 \pm 5$ & $72 \pm 4$ & $73 \pm 6$ \\
PQ interval (ms) & ALP & $109 \pm 9$ & $111 \pm 10$ & $124 \pm 11$ & $136 \pm 15$ & $140 \pm 10$ & $140 \pm 8$ & $139 \pm 9$ \\
& DFP & $106 \pm 13$ & $107 \pm 12$ & $113 \pm 14$ & $126 \pm 15$ & $132 \pm 10$ & $135 \pm 14$ & $136 \pm 10$ \\
QRS width (ms) & ALP & $62 \pm 6$ & $64 \pm 6$ & $63 \pm 4$ & $63 \pm 5$ & $62 \pm 6$ & $62 \pm 6$ & $62 \pm 6$ \\
& DFP & $62 \pm 5$ & $62 \pm 4$ & $62 \pm 4$ & $62 \pm 4$ & $63 \pm 5$ & $64 \pm 5$ & $63 \pm 5$ \\
QT interval (ms) & ALP & $277 \pm 16$ & $279 \pm 18$ & $282 \pm 20$ & $290 \pm 19$ & $309 \pm 22$ & $316 \pm 20$ & $325 \pm 28$ \\
& DFP & $271 \pm 22$ & $274 \pm 26$ & $262 \pm 20$ & $273 \pm 17$ & $298 \pm 19$ & $313 \pm 25$ & $322 \pm 29$ \\
& ALP & $35 \pm 4$ & $36 \pm 6$ & $37 \pm 4$ & $40 \pm 5$ & $42 \pm 6$ & $44 \pm 6$ & $47 \pm 4$ \\
& DFP & $33 \pm 4$ & $34 \pm 5$ & $33 \pm 5$ & $37 \pm 6$ & $40 \pm 7$ & $44 \pm 8$ & $46 \pm 8$ \\
\hline
\end{tabular}

Presented data were obtained from ECG recordings during normal sinus rhythm. Values are mean \pm SD. $\mathrm{n}=6$ for both amiodarone-loaded patch (ALP) and drug-free patch (DFP) groups. ${ }^{*} \mathrm{P}<0.05$ ALP vs DFP. 

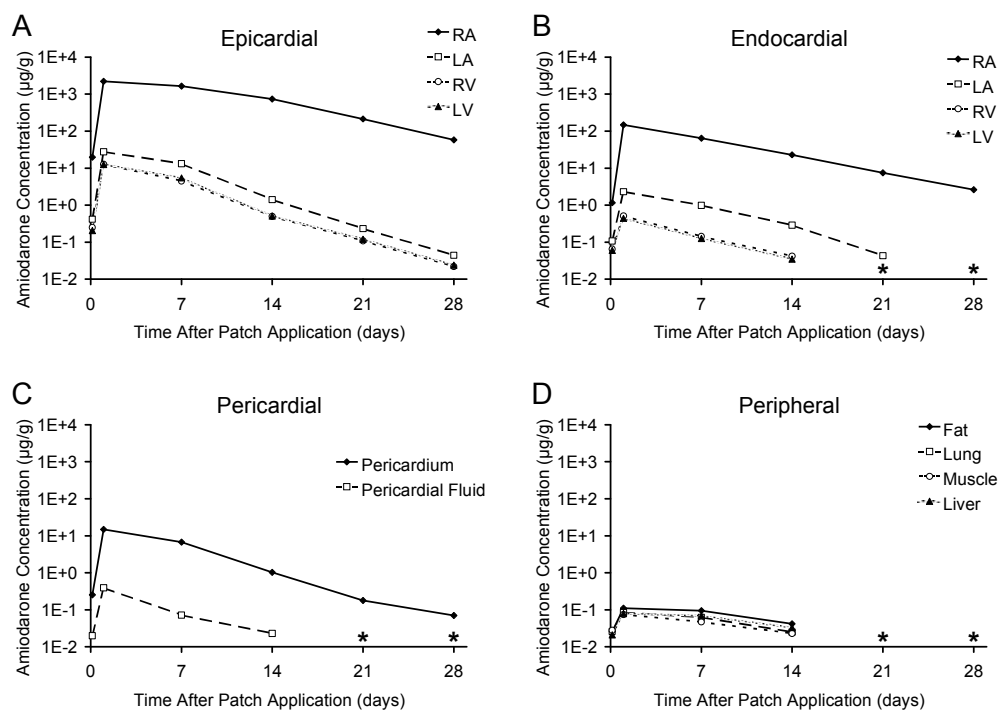

Figure 1. Amiodarone distribution. A, Epicardial amiodarone concentrations in right atrium (RA), left atrium (LA), right ventricle (RV) and left ventricle (LV). B, Endocardial amiodarone concentrations in RA, LA, RV and LV. C, Amiodarone concentrations in pericardium and pericardial fluid. D, Amiodarone concentrations in abdominal fat, the medial part of the cranial lobe of the right lung, skeletal muscle of the hind limb, and liver. Data were obtained 3 hours, and 1, 7, 14, 21 and 28 days after patch application; n=1 for each point in time. ${ }^{*}$ Amiodarone concentration below detection limit $(<10 \mathrm{ng} / \mathrm{ml})$.
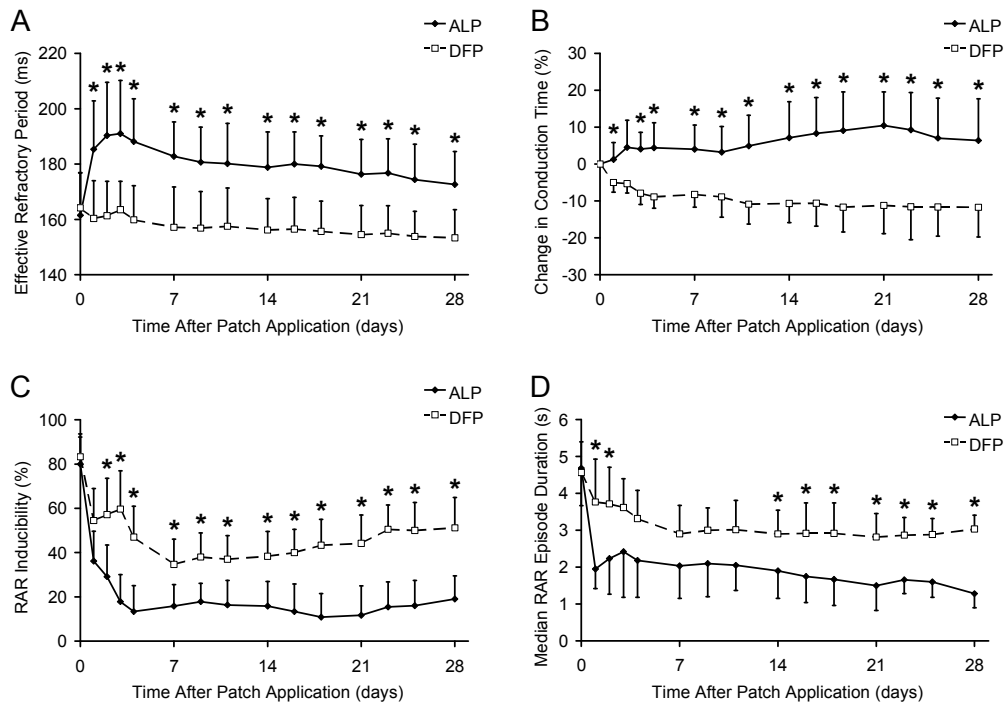

Figure 2. Atrial electrophysiology. A, Effective refractory period. B, Change in conduction time relative to baseline measurements. C, Rapid atrial response (RAR) inducibility. D, Median RAR duration. $n=6$ for both amiodarone-loaded patch (ALP) and drug-free patch (DFP) groups. ${ }^{*} P<0.05$. 


\section{Pharmacodynamic Feasibility: Antiarrhythmic Efficacy}

Effects of patch application on atrial electrophysiology are shown in Figure 2. Application of amiodarone-loaded patches produced significant increases in right atrial ERPs and conduction times over the time period evaluated in the present study. The ERP in the ALP group was maximal at $192 \pm 19 \mathrm{~ms}$ at 3 days after patch application compared with $163 \pm 10 \mathrm{~ms}$ in the DFP group. At 28 days after patch application, the ERP was still $20 \mathrm{~ms}$ higher in the ALP group. With respect to conduction times, no significant differences between groups were observed during baseline measurements. After application of amiodarone-loaded patches, conduction times tended to prolong, with mean changes ranging from $+1.3 \%$ to $+10.5 \%$, whereas a shortening was observed after application of drug-free patches, with mean changes ranging from $-5.0 \%$ to $-11.7 \%$, producing significant differences between the ALP and DFP groups (except for day 2 after patch application).

Atrial burst pacing in both groups did not produce sustained (ie, $>60$ seconds) episodes of atrial fibrillation either at baseline measurements or follow-up. Instead, RARs, typically lasting several seconds, were observed (Figure 3). RAR inducibility was significantly decreased in the ALP group compared with that seen in the DFP group from days 2 to 28 after patch application (Figure 2, C). Durations of RAR episodes tended to be longer in the DFP group, but this only reached significance in the 2 days after application of drug-free patches and during the last 2 weeks of follow-up (Figure 2, D).

Amiodarone-loaded patch

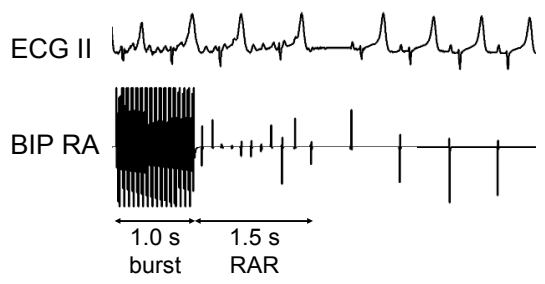

Drug-free patch

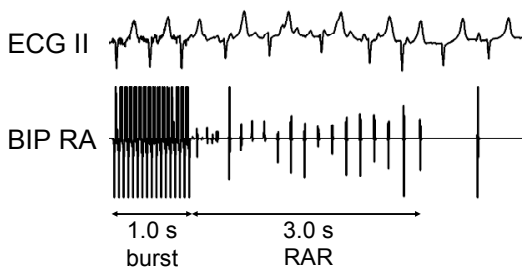

Figure 3. Typical responses to burst pacing 28 days after patch application. BIP RA indicates right atrial bipolar electrode; RAR, rapid atrial response. 


\section{DISCUSSION}

The main findings of this study are that epicardial application of bilayered amiodarone-eluting patches (1) is well tolerated, (2) yields persistently higher drug concentrations in the RA than in other cardiac and extracardiac tissues (by $2-4$ orders of magnitude), (3) increases ERPs and conduction times, and (4) decreases vulnerability to burst pacing-induced atrial tachyarrhythmias for at least 28 days.

Two decades ago, lidocaine-releasing polyurethane matrices were studied for the treatment of ventricular fibrillation..$^{12}$ The aim of developing these drug-delivery systems was to achieve sustained therapeutic myocardial concentrations at reduced systemic drug concentrations, minimizing the risk for unwanted extracardiac effects. Polyurethane matrices were subsequently loaded with propanolol, verapamil, and ibutilide, all demonstrating successful local drug delivery for several hours in animal studies. ${ }^{13-15}$ However, polyurethane is nonbiodegradable, which limits its clinical use as a matrix for epicardial drug delivery. One study investigated the long-term effects of sotalol controlled release implants, including polyurethane and silicone matrices applied to the ventricular epicardium and intrapericardially infused biodegradable poly(lactic-co-glycolic acid) microspheres. ${ }^{9}$ Thirty days after poly(lactic-co-glycolic acid) microsphere delivery, atrial drug concentrations were similar to ventricular drug concentrations, whereas 60 days after silicone matrix application, sotalol levels between matrix-covered and noncovered ventricular myocardium were equal. These results indicate that the use of drug delivery systems that only consist of drug-loaded matrices do not allow targeting of one specific myocardial region. The most likely mechanism is drug release from the matrix into the pericardial fluid, which facilitates rapid drug distribution to other heart chambers. However, other transport mechanisms (eg, through subepicardial lymphatics or vascular plexi) might also contribute to pan-cardiac drug distribution.

In the present study a biodegradable bilayered patch was used, consisting of an amiodarone-loaded matrix facing the epicardium and an impermeable backing layer facing the pericardium. The latter minimizes drug release into the pericardial fluid, thereby slowing distribution to other cardiac regions and enabling longerlasting site-specific drug delivery. Indeed, this seems to be reflected by the very high amiodarone concentrations in the epicardium of the RA compared with epicardial levels in other heart chambers up to 28 days after patch application. Regarding endocardial amiodarone concentrations, which were lower than epicardial drug concentrations, these regional differences appeared to be somewhat smaller, probably because of drug distribution through the lymphatics and blood vessels, different diffusion characteristics of the cardiac tissue layers, or both. After burst release in the first 24 hours after application, patches yielded monoexponentially decreasing amiodarone concentrations over the next 4 weeks. Epicardially applied 
PEG-based matrices with thioester bonds degrade within several weeks, indicating that the monoexponential decrease of drug levels was probably both diffusion and degradation mediated. ${ }^{16}$ A PLCL matrix was selected as the backing layer because this copolymer degrades much slower, on the order of 6 to 12 months, ensuring preserved impermeability during amiodarone release from the PEG-based matrix. ${ }^{17}$

Previous studies used the pericardial sac as a drug reservoir to achieve local amiodarone delivery for several hours. Intrapericardial infusion in dogs resulted in high tissue amiodarone concentrations in atria and the ventricular epicardium, whereas plasma only contained trace drug concentrations. ${ }^{6}$ In addition, atrial vulnerability to electrically induced tachyarrhythmias was decreased. In pigs it was shown that the higher atrial uptake of amiodarone, in contrast to epicardial ventricular uptake, was positively related to the intrapericardially delivered dose. ${ }^{7}$ In a recent study in goats, we observed transmural amiodarone concentration gradients with high epicardial drug levels in both atria after intrapericardial infusion that nevertheless produced similar epicardial and endocardial antiarrhythmic efficacy. ${ }^{8}$ Intravenous infusion of an equal amiodarone dose resulted in significantly lower antiarrhythmic efficacy both epicardially and endocardially. The present study demonstrates atrium-specific amiodarone delivery during a much longer period than seen in previous intrapericardial infusion studies. Amiodarone-loaded patches produced significant antiarrhythmic effects up to 28 days after application, whereas plasma drug levels remained less than $10 \mathrm{ng} / \mathrm{ml}$ (detection limit), which is more than 100 times lower than therapeutic plasma levels during intravenous or chronic oral amiodarone administration. Peak amiodarone concentrations in extracardiac tissues, such as fat, lung, skeletal muscle, and liver, were 2 to 4 orders of magnitude lower than levels observed during long-term oral amiodarone therapy. ${ }^{18}$

Amiodarone has multiple electrophysiologic effects, including class I, II, III, and IV antiarrhythmic actions. ${ }^{19}$ In the present study atrial ERPs were maximally prolonged in the first days after patch application, whereas effects on conduction times were maximal 2 weeks later, despite decreasing atrial amiodarone concentrations. The latter can be explained by treatment duration-dependent changes in amiodarone's ionic current blocking profile or by postoperative factors simultaneously affecting conduction time. The presence of surgical intervention-related influences on atrial electrophysiology was apparent in animals treated with drug-free patches, showing a peak of atrial arrhythmic vulnerability on days 2 and 3 after thoracotomy, which coincides with the peak incidence of POAF. ${ }^{20}$ Treatment with amiodarone-loaded patches significantly attenuated atrial vulnerability to burst pacinginduced tachyarrhythmias. Therefore efficacy of this new technique in preventing POAF is anticipated. QT intervals and maximal $\mathrm{T}_{\text {peak- }} \mathrm{T}_{\text {end }}$ intervals did not differ between the ALP and DFP groups, indicating low risk of drug-induced ventricular arrhythmias. 
In addition to POAF, epicardial polymeric release systems have also been investigated for other clinical applications, requiring delivery of substances with a higher molecular weight than amiodarone. A PEGylated fibrin patch was used to deliver the chemokine stromal cell-derived factor-1 alpha to ventricular infarct areas of mice. ${ }^{21}$ Transmural atrial gene transfer in pigs was achieved by means of epicardial release of adenoviral vectors from a poloxamer gel..$^{22}$ Growth factor-supplemented collagen matrices enhanced survival of transplanted cardiomyoblasts in ischemic rat hearts. ${ }^{23}$ These examples show the versatility of polymeric release systems, enabling advances in cardiovascular drug therapy, gene therapy, and cell therapy. ${ }^{24}$

\section{Limitations}

One important difference with human subjects is that the caprine atrial action potential is shorter. ${ }^{25}$ In addition, the atrial structure of patients with cardiovascular disease is likely to be different from the atrial structure of healthy goats. Therefore extrapolating the results of the present study to human subjects must be done with caution.

The follow-up period for this study lasted 28 days after patch application. Although this period is sufficiently long lasting for prevention of POAF, it would be interesting to study drug distribution and electrophysiologic effects for even longer periods. Patches capable of sustained delivery of amiodarone for several months might also be used in the treatment of paroxysmal atrial fibrillation. Minimally invasive access to the normal pericardial cavity might further promote the clinical applicability of epicardial polymeric release systems.

Animals in the present study did not experience sustained episodes of atrial fibrillation. Additional studies in representative animal models are required to evaluate the efficacy of local amiodarone delivery in the treatment of chronic atrial fibrillation. Epicardial and endocardial electrophysiologic mapping might provide further insight into antiarrhythmic mechanisms of epicardial amiodarone delivery.

Patches were applied only to the RA to evaluate the feasibility of sustained targeted drug delivery to a specific heart chamber. The RA was chosen to determine the negative chronotropic effects of patch application near the sinoatrial node. R-R intervals briefly increased after patch application, but no significant sinus bradycardia was observed. In future clinical application, patches should be applied to both the RA and LA.

Epicardially placed PEG-based biomaterials are currently used for prevention of pericardial adhesions after cardiac surgery. ${ }^{16}$ However, individual hypersensitivity to amiodarone, iodine, or patch degradation products might cause local inflammation and fibrosis after patch application. Further studies are needed to evaluate the safety of this method. 
The drug-loaded matrix showed minimal adhesive properties, necessitating suturing of the patch to the atrial epicardium. Future development of a self-adhesive patch by tailoring PEG matrix characteristics could ease application and prevent needle-induced microtrauma to the atrium.

\section{Conclusions}

Atrium-targeted delivery of amiodarone is feasible through epicardial application of a bilayered patchwith anamiodarone-loaded matrix facing the epicardium and an impermeable backing layer facing the pericardium. Sustained atrial antiarrhythmic effects up to 1 month after patch application can be achieved, whereas tissue drug concentrations in ventricles and extracardiac tissues remain several orders of magnitude lower than atrial drug levels. These results indicate that this method might be promising to prevent POAF with a lower risk for ventricular and systemic side effects compared with intravenous and oral amiodarone administration. 


\section{REFERENCES}

1. Hogue CW, Jr., Creswell LL, Gutterman DD, et al. Epidemiology, mechanisms, and risks: American College of Chest Physicians guidelines for the prevention and management of postoperative atrial fibrillation after cardiac surgery. Chest. 2005;128(2 Suppl):9S-16S.

2. Echahidi N, Pibarot P, O'Hara G, et al. Mechanisms, prevention, and treatment of atrial fibrillation after cardiac surgery. J Am Coll Cardiol. 2008;51(8):793-801.

3. Fuster V, Ryden LE, Cannom DS, et al. ACC/AHA/ESC 2006 Guidelines for the Management of Patients with Atrial Fibrillation: a report of the American College of Cardiology/American Heart Association Task Force on Practice Guidelines and the European Society of Cardiology Committee for Practice Guidelines (Writing Committee to Revise the 2001 Guidelines for the Management of Patients With Atrial Fibrillation): developed in collaboration with the European Heart Rhythm Association and the Heart Rhythm Society. Circulation. 2006;114(7):e257354.

4. Camm AJ. Safety considerations in the pharmacological management of atrial fibrillation. Int J Cardiol. 2008;127(3):299-306.

5. Mitchell LB, Exner DV, Wyse DG, et al. Prophylactic oral amiodarone for the prevention of arrhythmias that begin early after revascularization, valve replacement, or repair: PAPABEAR: a randomized controlled trial. JAMA. 2005;294(24):3093-3100.

6. Ayers GM, Rho TH, Ben-David J, et al. Amiodarone instilled into the canine pericardial sac migrates transmurally to produce electrophysiologic effects and suppress atrial fibrillation. J Cardiovasc Electrophysiol. 1996;7(8):713-721.

7. Darsinos JT, Karli JN, Samouilidou EC, et al. Distribution of amiodarone in heart tissues following intrapericardial administration. Int J Clin Pharmacol Ther. 1999;37(6):301-306.

8. Bolderman RW, Hermans JJ, Rademakers LM, et al. Intrapericardial delivery of amiodarone and sotalol: atrial transmural drug distribution and electrophysiological effects. J Cardiovasc Pharmacol. 2009;54(4):355-363.

9. Labhasetwar V, Underwood T, Gallagher M, et al. Sotalol controlled-release systems for arrhythmias: in vitro characterization, in vivo drug disposition, and electrophysiologic effects. $J$ Pharm Sci. 1994;83(2):156-164.

10. Dias AA, Boerakker M, Nijenhuis AJ. Polymers comprising thioester bonds. PCT/EP.2006/008730.

11. Bolderman RW, Hermans JJ, Maessen JG. Determination of the class III antiarrhythmic drugs dronedarone and amiodarone, and their principal metabolites in plasma and myocardium by high-performance liquid chromatography and UV-detection. J Chromatogr B Analyt Technol Biomed Life Sci. 2009;877(18-19):1727-1731.

12. Sintov A, Scott W, Dick M, et al. Cardiac controlled release for arrhythmia therapy: Lidocainepolyurethane matrix studies. Journal of Controlled Release. 1988;8(2):157-165.

13. Siden R, Flowers WE, Levy RJ. Epicardial propranolol administration for ventricular arrhythmias in dogs: matrix formulation and characterization. Biomaterials. 1992;13(11):764-770.

14. Siden R, Kadish A, Flowers W, et al. Epicardial controlled-release verapamil prevents ventricular tachycardia episodes induced by acute ischemia in a canine model. J Cardiovasc Pharmacol. 1992;19(5):798-809.

15. Labhasetwar V, Underwood $T$, Heil RW, Jr., et al. Epicardial administration of ibutilide from polyurethane matrices: effects on defibrillation threshold and electrophysiologic parameters. $J$ Cardiovasc Pharmacol. 1994;24(5):826-840. 
16. Hendrikx M, Mees U, Hill AC, et al. Evaluation of a novel synthetic sealant for inhibition of cardiac adhesions and clinical experience in cardiac surgery procedures. Heart Surg Forum. 2001;4(3):204-210.

17. Pitt CG, Gratzl MM, Kimmel GL, et al. Aliphatic polyesters II. The degradation of poly (DLlactide), poly (epsilon-caprolactone), and their copolymers in vivo. Biomaterials. 1981;2(4):215220.

18. Adams PC, Holt DW, Storey GC, et al. Amiodarone and its desethyl metabolite: tissue distribution and morphologic changes during long-term therapy. Circulation. 1985;72(5):1064-1075.

19. Kodama I, Kamiya K, Toyama J. Cellular electropharmacology of amiodarone. Cardiovasc Res. 1997;35(1):13-29.

20. Mathew JP, Fontes ML, Tudor IC, et al. A multicenter risk index for atrial fibrillation after cardiac surgery. JAMA. 2004;291(14):1720-1729.

21. Zhang G, Nakamura $Y$, Wang $X$, et al. Controlled release of stromal cell-derived factor- 1 alpha in situ increases c-kit+ cell homing to the infarcted heart. Tissue Eng. 2007;13(8):2063-2071.

22. Kikuchi K, McDonald AD, Sasano T, et al. Targeted modification of atrial electrophysiology by homogeneous transmural atrial gene transfer. Circulation. 2005;111(3):264-270.

23. Kutschka I, Chen IY, Kofidis T, et al. Collagen matrices enhance survival of transplanted cardiomyoblasts and contribute to functional improvement of ischemic rat hearts. Circulation. 2006;114(1 Suppl):I167-173.

24. Spadaccio $C$, Chello $M$, Trombetta $M$, et al. Drug releasing systems in cardiovascular tissue engineering. J Cell Mol Med. 2009;13(3):422-439.

25. Franz MR, Karasik PL, Li C, et al. Electrical remodeling of the human atrium: similar effects in patients with chronic atrial fibrillation and atrial flutter. J Am Coll Cardiol. 1997;30(7):1785-1792. 


\section{CHAPTER 6}

Effects of epicardial amiodarone delivery on transmural atrial electrophysiology and sustained atrial fibrillation 


\section{Abstract}

Background: Epicardial delivery of amiodarone through polymeric release systems has been shown to produce therapeutic myocardial drug concentrations, while systemic drug levels are minimized. The aim of this pilot study was to determine the effects of epicardial application of an amiodarone-releasing hydrogel on transmural atrial electrophysiology in the goat model of sustained atrial fibrillation (AF).

Methods: Eight goats were chronically instrumented with right atrial endocardial electrodes. AF was maintained for four weeks by repetitive burst pacing. Simultaneous endo-epicardial high-density mapping (electrode spacing $1.6 \mathrm{~mm}$ ) was performed in left atrial free walls before and after epicardial application of a poly(ethylene glycol)-based hydrogel loaded with amiodarone (1 mg/kg bodyweight) $(n=5)$ or without drug $(n=3)$.

Results: Amiodarone-loaded hydrogels produced a non-significant prolongation of both epicardial and endocardial atrial effective refractory periods. Epicardial drug application tended to decrease epi- and endocardial atrial conduction velocities. Stability of AF (expressed as the ratio of AF-paroxysm-duration [s] devided by the number of AF-induction attempts [a]) was reduced in amiodarone-treated animals (from 296 to 172 s/a, $P=0.068$ ), whereas an increase was observed in the control group (from 184 to 404 s/a, n=3).

Conclusions: Epicardial delivery of amiodarone via application of a hydrogel release system produces antiarrhythmic effects in both epicardium and endocardium. The results of this pilot study suggest that epicardial amiodarone administration may reduce AF stability in the goat model of sustained AF. 


\section{INTRODUCTION}

Pharmacotherapy of cardiac disease is often accompagnied by systemic side effects. This also holds true for antiarrhythmic treatment of atrial fibrillation (AF), the most common cardiac arrhythmia requiring medical attention. Management principles of AF involve prevention of thromboembolism, and either a rate or rhythm control strategy. ${ }^{1-3}$ The choice between the latter two strategies has been the subject of extensive debate, which is in part due to the moderate effectiveness and proarrhythmic characteristics of currently available drugs for rhythm control.

In order to improve drug efficacy and minimize adverse effects, an alternative approach may be adopted, namely local drug delivery. The heart is surrounded by the pericardial cavity, which is ideally suited for local cardiac pharmacotherapy, because it provides a natural drug reservoir and space for epicardial application of drug delivery systems. In the past two decades both intrapericardial infusion of drug solutions and application of polymeric release systems have been studied to achieve local antiarrhythmic therapy.-18

Two previous studies investigated efficacy of epicardial pharmacotherapy in animal models of sustained AF. Intrapericardial ibutilide administration in dogs with pacing-induced AF did not result in higher AF termination rates compared with intrapericardial saline, despite significant atrial electrophysiological effects. ${ }^{13}$ In the goat model of persistent $\mathrm{AF}$, intrapericardial sotalol and flecainide infusion predominantly affected atrial electrophysiology in the epicardium. ${ }^{15}$ However, intrapericardial administration did not terminate AF to a greater extent than intravenous infusion, possibly due to subtherapeutic endocardial drug levels or enhancement of potentially arrhythmogenic heterogeneity in transmural electrophysiology.

In a recent study we showed that intrapericardial delivery of the multi-ion channel-blocker amiodarone may influence atrial transmural electrophysiology more homogeneously, resulting in a reduction of AF-inducibility in atria of healthy goats. ${ }^{16}$ In addition, feasibility of long-term atrium-targeted delivery of amiodarone was demonstrated using biodegradable polymeric release formulations. ${ }^{17,18}$ No data are currently available on effects of epicardial amiodarone delivery on sustained AF. Furthermore, to the best of our knowledge, simultaneous endo-epicardial highdensity mapping during epicardial delivery of antiarrhythmic drugs has not been performed so far.

The first aim of the present study was to investigate effects of epicardial application of an amiodarone-releasing hydrogel on epicardial and endocardial atrial electrophysiology. Second, this study aimed to evaluate efficacy of epicardial amiodarone treatment in the goat model of sustained AF. 


\section{Methods}

All procedures conformed to the "Guidelines for the Care and Use of Laboratory Animals" published by the National Institutes of Health. In addition, the local ethical Animal Studies Committee of the University of Maastricht approved this study protocol. Eight domestic goats (weight $40-65 \mathrm{~kg}$ ) were randomly allocated to a drugfree hydrogel group (DFH group, $n=3$ ) or an amiodarone-loaded hydrogel group (ALH group, $\mathrm{n}=5$ ).

\section{Atrial Fibrillation Model}

Sustained AF was induced in all animals using the previously-reported goat model of artificially-maintained AF. ${ }^{19}$ During general anesthesia (induction: thiopental 20 $\mathrm{mg} / \mathrm{kg}$; maintenance: sufentanyl $6 \mu \mathrm{g} / \mathrm{kg} / \mathrm{h}$, midazolam $0.8 \mathrm{mg} / \mathrm{kg} / \mathrm{h}$ ) the jugular vein was dissected and a bipolar pacing electrode (Medtronic, Capsurefix ${ }^{\circledR}$ ) was placed in the body of the right atrium under fluoroscopic guidance. The distal end of the pacing lead was connected to a neurostimulator (Medtronic, Itrel1 ${ }^{\circledR}$ ). After 2 weeks of recovery from implantation, AF was maintained for 4 weeks by repetitive $50-\mathrm{Hz}$ burst pacing at three times threshold every other second.

\section{Experimental Procedure}

Anesthesia was induced by thiopental $20 \mathrm{mg} / \mathrm{kg}$ and maintained by a combination of sufentanyl $(6 \mu \mathrm{g} / \mathrm{kg} / \mathrm{h})$, midazolam $(0.8 \mathrm{mg} / \mathrm{kg} / \mathrm{h})$ and pancuronium $(0.3$ $\mathrm{mg} / \mathrm{kg} / \mathrm{h}$ ), during ventilation with a 1:1 mixture of oxygen and room air. Limb-lead electrocardiogram, arterial blood pressure, temperature, end-expiratory $\mathrm{CO}_{2}$, and oxygen saturation (pulse oximeter) were monitored throughout the procedure. Saline $(0.9 \%, 5-8 \mathrm{ml} / \mathrm{kg} / \mathrm{h})$ was infused via a peripheral venous catheter to compensate for fluid loss.

A left intercostal thoracotomy was performed, and the left side of the heart was exposed. Two silicone patches of $5 \times 10 \mathrm{~mm}$, each containing 2 silver unipolar electrodes (1.5 mm diameter, $5 \mathrm{~mm}$ interelectrode distance), were sutured to the medial and lateral borders of the left atrial free wall. A third silicone electrode patch was fixed to the the anterior wall of the left ventricle, while a fourth (indifferent) electrode was placed in the thoracic cavity. A short incision was made in the left atrial free wall close to the atrio-ventricular groove. Then a high-density mapping device (spacing $1.6 \mathrm{~mm}$ ) for simultaneous endocardial and epicardial measurements was positioned in the left atrium (between to two atrial silicone electrode patches), using a purse-string suture to prevent blood leakage along the endocardial part of the device. $^{20}$ A custom-made 256-channel mapping amplifier (filtering bandwidth 0.1- 
$400 \mathrm{~Hz}$, sampling rate $1 \mathrm{kHz}, \mathrm{A} / \mathrm{D}$ resolution $16 \mathrm{bits}$ ) was used to record unipolar signals. Following baseline measurements, the epicardial part of the mapping device was held up, and the hydrogel (loaded with or without amiodarone) was sprayed over both the left and right atrial free walls (and over the left atrial silicone electrodes). After 5 minutes the device was closed again to make contact with the epicardial surface, and electrophysiological measurements were repeated after 1, 2, and 3 hours.

\section{Hydrogel Preparation}

CoSeal $^{\circledR}$ (Angiotech Pharmaceuticals, Vancouver, Canada), a two-component surgical sealant based on biodegradable poly(ethylene glycol) polymers, was obtained from Baxter Healthcare (Fremont, CA, USA). After mixing the poly(ethylene glycol) powder with both solutions, a hydrogel is formed that attaches to tissues. All hydrogels were made immediately before application. Drug-free hydrogels were prepared according to the instructions for use. Amiodarone-loaded hydrogels were obtained by adding amiodarone hydrochloride powder to the poly(ethylene glycol) powder before mixing with the solutions. The hydrogel end product contained an amiodarone concentration of $25 \mathrm{mg} / \mathrm{ml}$. A total of $1 \mathrm{mg}$ amiodarone per $\mathrm{kg}$ bodyweight was applied to both atria (corresponding to a hydrogel volume of $40 \mu \mathrm{l}$ per kg bodyweight).

\section{Electrophysiological Measurements}

Cardioversion to sinus rhythm was achieved by applying internal DC shocks with two 6 F defibrillation catheters (Elecath, Rahway, NJ, USA) positioned in the coronary sinus and in the posterior right atrium, and connected to a defibrillator (Medtronic Lifepak $9 \mathrm{~B}^{\circledR}$ ). Atrial effective refractory periods (AERP) were measured during bipolar pacing at 4 times the threshold. Measurements were performed at the centres of the endocardial and epicardial electrode high-density arrays, and with both atrial silicone electrodes. Basic stimuli with cycle lengths of 250 and $400 \mathrm{~ms}$ (S1) were interpolated by single premature stimuli (S2). The S1-S2 interval was incremented in 2-millisecond steps and the longest S1-S2 interval that failed to capture was defined as the AERP. ${ }^{21}$

Epicardial and endocardial high-density electrogram maps were computed with custom made software. Local activation times were marked in each unipolar electrogram at the maximum negative $\mathrm{d} V / \mathrm{d} t$. Conduction times were calculated by taking the differences between electrode activation times. Epicardial and endocardial atrial conduction velocities were measured during regular pacing with intervals ranging from 200 to $400 \mathrm{~ms}$ either from the medial or the lateral atrial silicone elec- 
trode. The conduction velocities were calculated from the conduction times recorded between the medial and lateral borders of both epicardial and endocardial high-density arrays.

To assess stability of AF, burst pacing $(1 \mathrm{~s}, 50 \mathrm{~Hz})$ was applied using the endocardial right atrial pacing lead. When an induced AF-episode lasted more than 10 minutes, electrical cardioversion to sinus rhythm was performed as described previously. This was repeated for a maximum of 20 AF-induction attempts, or until the cumulative duration of AF-episodes reached 30 minutes.

\section{Statistical Analysis}

Data are expressed as mean \pm SD unless otherwise mentioned. Due to the small group size in this pilot study, statistical differences between groups were not calculated. Effects within the ALH-group were calculated by Wilcoxon signed rank tests. A 2-tailed $P$ value of $<0.05$ was considered statistically significant.

\section{RESULTS}

All animals had sustained atrial fibrillation at the beginning of the experimental procedure and could be succesfully converted to normal sinus rhythm by applying internal DC shocks at the start of electrophysiological measurements. Epicardial application of hydrogel produced a marked increase in the pacing threshold of the epicardial part of the high-density electrode array, prohibiting local AERP measurements in 5 of 8 animals. In the 3 other animals, epicardial array pacing thresholds increased 2, 5 and 6 times after hydrogel application compared to baseline measurements. In addition, capture at the endocardial electrode array was lost in 3 of 8 animals. With respect to the other atrial electrodes (epicardial silicone patches and endocardial right atrial lead), pacing thresholds remained stable during the course of the experiments.

\section{Atrial Refractoriness}

Effects of epicardial hydrogel application on AERP are shown in Table 1 . In the experiments with amiodarone-loaded hydrogel in which endocardial AERP could be measured before and after hydrogel application, an increase was observed (from $108 \pm 29$ to $156 \pm 42 \mathrm{~ms}, \mathrm{n}=3$ ). Mean epicardial AERP prolonged from $109 \pm 16 \mathrm{~ms}$ at baseline, to $122 \pm 16 \mathrm{~ms}$ after drug administration $(n=5, P=0.080)$. In the DFH group effects on endocardial AERP were mixed (both increase and decrease), while mean epicardial AERP tended to shorten (from $151 \pm 23$ to $142 \pm 30 \mathrm{~ms}$ ). Due to loss of pacing capture at the high-density arrays (especially at the epicardial part), exactly- 
opposed measurement of epicardial and endocardial AERP after hydrogel application could only be performed in one animal of each group (experiments 1 and 7 in Table 1).

Table 1. Atrial Refractoriness

\begin{tabular}{|c|c|c|c|c|c|c|c|c|c|c|}
\hline & \multirow[t]{2}{*}{$\begin{array}{l}\text { Experiment } \\
\text { number }\end{array}$} & \multirow{2}{*}{$\begin{array}{c}\text { Basic } \\
\text { stimulus } \\
\text { cycle } \\
\text { length }\end{array}$} & \multicolumn{2}{|c|}{$\begin{array}{l}\text { AERP: center of } \\
\text { endocardial array }\end{array}$} & \multicolumn{2}{|c|}{$\begin{array}{l}\text { AERP: center of } \\
\text { epicardial array }\end{array}$} & \multicolumn{2}{|c|}{$\begin{array}{l}\text { AERP: medial } \\
\text { epicardial bipolar }\end{array}$} & \multicolumn{2}{|c|}{$\begin{array}{c}\text { AERP: lateral } \\
\text { epicardial bipolar }\end{array}$} \\
\hline & & & Baseline & Hydrogel & Baseline & Hydrogel & Baseline & Hydrogel & Baseline & Hydrogel \\
\hline \multirow{10}{*}{$\begin{array}{l}\text { ALH } \\
\text { group }\end{array}$} & \multirow[t]{2}{*}{1} & $400 \mathrm{~ms}$ & 79 & 96 & 84 & 118 & 94 & 112 & 86 & 110 \\
\hline & & $250 \mathrm{~ms}$ & 80 & 108 & 84 & 116 & 92 & 104 & 92 & 108 \\
\hline & \multirow[t]{2}{*}{2} & $400 \mathrm{~ms}$ & 190 & $\mathrm{nc}$ & $\mathrm{nc}$ & $\mathrm{nc}$ & 140 & 156 & 100 & 106 \\
\hline & & $250 \mathrm{~ms}$ & 180 & nc & nc & nc & 138 & 138 & 108 & 122 \\
\hline & \multirow[t]{2}{*}{3} & $400 \mathrm{~ms}$ & 126 & 178 & 120 & nc & 92 & 122 & 90 & 140 \\
\hline & & $250 \mathrm{~ms}$ & 152 & 190 & 124 & nc & 102 & 130 & 152 & 140 \\
\hline & \multirow[t]{2}{*}{4} & $400 \mathrm{~ms}$ & 136 & nc & 148 & nc & 114 & 148 & 114 & 110 \\
\hline & & $250 \mathrm{~ms}$ & 160 & $\mathrm{nc}$ & 162 & nc & 126 & 160 & 130 & 126 \\
\hline & \multirow[t]{2}{*}{5} & $400 \mathrm{~ms}$ & 92 & 172 & 96 & nc & 100 & 104 & 92 & 74 \\
\hline & & $250 \mathrm{~ms}$ & 120 & 190 & 98 & nc & 122 & 128 & 98 & 86 \\
\hline \multirow{6}{*}{$\begin{array}{l}\text { DFH } \\
\text { group }\end{array}$} & \multirow[t]{2}{*}{6} & $400 \mathrm{~ms}$ & $\mathrm{nc}$ & $\mathrm{nc}$ & 202 & 206 & 142 & 136 & 174 & 156 \\
\hline & & $250 \mathrm{~ms}$ & nc & nc & 196 & 192 & 154 & 144 & 178 & 154 \\
\hline & \multirow[t]{2}{*}{7} & $400 \mathrm{~ms}$ & 110 & 150 & 160 & 166 & 148 & 122 & 164 & 174 \\
\hline & & $250 \mathrm{~ms}$ & 122 & 164 & 154 & 176 & 146 & 130 & 162 & 176 \\
\hline & \multirow[t]{2}{*}{8} & $400 \mathrm{~ms}$ & 134 & 114 & 138 & nc & 148 & 118 & 90 & 84 \\
\hline & & $250 \mathrm{~ms}$ & 150 & 130 & 150 & $\mathrm{nc}$ & 160 & 122 & 98 & 88 \\
\hline
\end{tabular}

ALH, amiodarone-loaded hydrogel; DFH, drug-free hydrogel; AERP, Atrial Effective Refractory Period in milliseconds; nc, no capture during pacing.

\section{Atrial Conduction Velocity}

In three experiments (2 in the ALH group and 1 in the DFH group) conduction velocities could not be determined due to severe fractionation of both epicardial and endocardial electrograms. Results of the other experiments are listed in Table 2. Mean epicardial and endocardial conduction velocities decreased in the amiodarone-treated animals with mean values of $78 \pm 4$ and $91 \pm 10 \mathrm{~cm} / \mathrm{s}$ at baseline, respectively, and $74 \pm 6$ and $80 \pm 9 \mathrm{~cm} / \mathrm{s}$ after hydrogel application $(\mathrm{n}=3)$. In the two animals of the DFH group in which conduction velocity could be measured, a small increase was observed $(73 \pm 9$ and $73 \pm 14 \mathrm{~cm} / \mathrm{s}$ at baseline, for epicardium and endocardium respectively, and $78 \pm 8$ and $76 \pm 10 \mathrm{~cm} / \mathrm{s}$ after hydrogel application). 
Table 2. Atrial Conduction Velocity

\begin{tabular}{|c|c|c|c|c|c|c|c|c|c|c|}
\hline & \multirow[t]{2}{*}{$\begin{array}{l}\text { Experiment } \\
\text { number }\end{array}$} & \multirow{2}{*}{$\begin{array}{c}\text { Basic } \\
\text { stimulus } \\
\text { cycle } \\
\text { length } \\
\end{array}$} & \multicolumn{2}{|c|}{$\begin{array}{l}\text { Epicardial ACV } \\
\text { (medial to lateral) }\end{array}$} & \multicolumn{2}{|c|}{$\begin{array}{l}\text { Epicardial ACV } \\
\text { (lateral to medial) }\end{array}$} & \multicolumn{2}{|c|}{$\begin{array}{l}\text { Endocardial ACV } \\
\text { (medial to lateral) }\end{array}$} & \multicolumn{2}{|c|}{$\begin{array}{l}\text { Endocardial ACV } \\
\text { (lateral to medial) }\end{array}$} \\
\hline & & & Baseline & Hydrogel & Baseline & Hydrogel & Baseline & Hydrogel & Baseline & Hydrogel \\
\hline \multirow{9}{*}{$\begin{array}{l}\text { ALH } \\
\text { group }\end{array}$} & \multirow[t]{3}{*}{1} & $400 \mathrm{~ms}$ & 77 & 73 & 81 & 85 & 103 & 74 & 97 & 96 \\
\hline & & $350 \mathrm{~ms}$ & 70 & 78 & 81 & 83 & 96 & 85 & 97 & 96 \\
\hline & & $200 \mathrm{~ms}$ & 72 & 80 & 81 & 80 & 96 & 89 & 93 & 87 \\
\hline & \multirow[t]{3}{*}{3} & $400 \mathrm{~ms}$ & 70 & $\mathrm{mv}$ & 77 & 72 & 103 & 90 & 90 & 80 \\
\hline & & $350 \mathrm{~ms}$ & 72 & $\mathrm{mv}$ & 80 & 70 & 111 & 85 & 90 & 80 \\
\hline & & $200 \mathrm{~ms}$ & 70 & $\mathrm{mv}$ & 72 & 70 & 90 & 80 & 85 & 72 \\
\hline & \multirow[t]{3}{*}{5} & $400 \mathrm{~ms}$ & 77 & 74 & $\mathrm{mv}$ & 88 & 90 & 80 & $\mathrm{mv}$ & 72 \\
\hline & & $350 \mathrm{~ms}$ & 77 & 74 & $\mathrm{mv}$ & 86 & 85 & 72 & $\mathrm{mv}$ & 72 \\
\hline & & $200 \mathrm{~ms}$ & 86 & 62 & $\mathrm{mv}$ & 77 & 69 & 63 & $\mathrm{mv}$ & 65 \\
\hline \multirow{6}{*}{$\begin{array}{l}\text { DFH } \\
\text { group }\end{array}$} & \multirow[t]{3}{*}{7} & $400 \mathrm{~ms}$ & $\mathrm{mv}$ & 86 & $\mathrm{mv}$ & 98 & $\mathrm{mv}$ & 103 & $\mathrm{mv}$ & 85 \\
\hline & & $350 \mathrm{~ms}$ & 75 & 86 & 98 & 98 & 89 & 103 & 96 & 80 \\
\hline & & $200 \mathrm{~ms}$ & 73 & 71 & 83 & 90 & 85 & 85 & 80 & 76 \\
\hline & \multirow[t]{3}{*}{8} & $400 \mathrm{~ms}$ & 65 & 76 & 65 & 71 & 64 & 64 & 61 & 71 \\
\hline & & $250 \mathrm{~ms}$ & 67 & 73 & 65 & 71 & 64 & 64 & 61 & 71 \\
\hline & & $200 \mathrm{~ms}$ & 76 & 65 & 62 & 80 & 59 & 70 & 72 & 72 \\
\hline
\end{tabular}

$\mathrm{ALH}$, amiodarone-loaded hydrogel; DFH, drug-free hydrogel; ACV, Atrial Conduction Velocity in centimeters per second; mv, missing value. Experiment numbers 2, 4 and 6 were excluded due to severe fractionation.

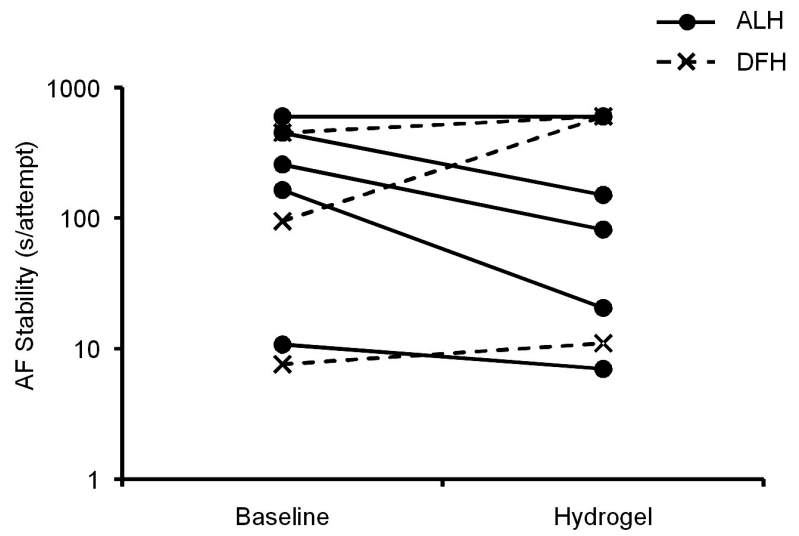

Figure 1. Atrial fibrillation stability. AF, Atrial fibrillation; AF Stability in seconds per attempt; ALH, amiodarone-loaded hydrogel; DFH, drug-free hydrogel. 


\section{Atrial Fibrillation Stability}

Because of experimental time-constraints, measurement of AF stability was limited to both cumulative AF duration (maximum 30 minutes) and number of AFinduction attempts (maximum 20). Therefore AF stability is expressed as a ratio of these two parameters (Figure 1). At baseline, sustained AF was difficult to reinduce in two animals (one in each group), with mean AF-durations of 8 and 11 seconds. In the other animals, sustained episodes of AF could be readily reinduced, with means ranging from 1.5 to 10 minutes. After application of amiodarone-loaded hydrogel, a decrease in AF stability was observed (from $296 \pm 233$ to $172 \pm 246$ s/attempt, $n=5$, $P=0.068$ ). In the DFH group an increase in AF stability was seen from $184 \pm 234$ at baseline to $404 \pm 340$ s/attempt after hydrogel application.

\section{DISCUSSION}

Local pharmacotherapy through epicardial delivery has been suggested as a means to increase drug efficacy and minimize adverse effects. Particularly the thin atria seem to be suitable for this delivery method, because of their high surface area/wall thickness ratio. Due to the relatively thick walls of the ventricles, only a small fraction of intrapericardially delivered drugs reaches the ventricular midmyocardial and endocardial layers. ${ }^{15,16}$ Atrial selectivity can be further promoted by epicardial application of polymeric drug delivery systems to the atria. ${ }^{17,18}$

Clinically, the most relevant disease of the atria is AF, which has a high incidence and is associated with increased morbidity and mortality.22 Several studies have investigated efficacy of epicardial delivery of antiarrhythmic drugs for the treatment of AF. Intrapericardially infused solutions of amiodarone and procainamide resulted in a decreased inducibility of AF in atria of healthy dogs, pigs and goats. ${ }^{8,11,16}$ However, intrapericardial administration of ibutilide, flecainide and sotalol in animal models of pacing-induced sustained AF did not result in higher AF termination rates, despite significant atrial electrophysiological effects. ${ }^{13,15}$

A reason for AF-treatment failure of epicardial antiarrhythmic drug delivery could be the presence of atrial transmural concentration gradients with high epicardial levels and low endocardial levels, which may be associated with subtherapeutic endocardial effects and a potentially arrhythmogenic transmural gradient in refractoriness. ${ }^{15,16}$ The latter could particularly hold true for atria with structural remodeling, where electrical uncoupling may occur between the epicardium and endocardial atrial bundle network. ${ }^{20}$

In the present study simultaneous endo-epicardial measurements were conducted to evaluate effects of epicardial drug delivery on transmural electrophysiol- 
ogy. Results show that application of an amiodarone-releasing hydrogel may be associated with therapeutic efficacy in atria with structural remodeling. We observed that epicardial delivery of amiodarone tends to increase AERP and decrease atrial conduction velocity, not only at the atrial epicardium, but, interestingly, also at the atrial endocardium. Recently, we showed that application of amiodaronereleasing hydrogel to the atrium produced 10-fold higher epicardial drug concentrations than endocardial concentrations. ${ }^{18}$ However, present results point to a trend of a larger electrophysiological effect on the endocardium than on the epicardium. This seemingly inconsistent relationship between transmural amiodarone concentrations and transmural electrophysiological effects was also reported in two previous studies in which amiodarone was administered intrapericardially. Ayers et al demonstrated similar effects on epicardial and endocardial ERP in the right ventricle, despite 10-fold drug concentration differences between epicardium and endocardium. ${ }^{8}$ A previous study in our laboratory showed an increase in endocardial AERP of approximately $50 \%$ of the epicardial AERP increase, despite much lower endocardial amiodarone concentrations. ${ }^{16}$ These differences can only partly be explained by achievement of maximal drug effects at the epicardium due to very high amiodarone levels.

An alternative explanation for the inconsistent concentration-effect relationship might be transmural heterogeneity in atrial ionic currents, which implies that transmural drug effects not only depend on local drug concentrations, but also on local ionic current density.23-25 A recent study showed a 22-ms longer endocardial AERP than epicardial AERP in goats, providing further evidence for the presence of transmural heterogeneity of repolarizing ionic currents in the atria. ${ }^{20}$ With respect to the ventricles, increased transmural electrophysiological heterogeneity has been demonstrated to increase risk of ventricular arrhythmias. ${ }^{26}$ However, endoepicardial differences in refractoriness do not seem to play a role in the enhancement of atrial endo-epicardial electrical dispersion with increasing duration of AF. ${ }^{20}$

It remains a question whether changes in atrial transmural dispersion of refractoriness induced by epicardial drug delivery may increase or decrease stability of atrial arrhythmias. In the present study, epicardial application of hydrogel resulted in frequent loss of pacing capture, which hindered measurement of epicardial and endocardial AERP with precisely aligned electrode pairs. Although epicardial AERP measurements were also performed with bipolar electrodes at the medial and lateral borders of the left atrium, the epicardial surface beneath these electrode patches was not exposed to hydrogel, possibly leading to an underestimation of the amiodarone effect on epicardial AERP. Therefore, these results do not provide a definitive answer to the question whether efficacy of epicardially delivered amiodarone for the treatment of AF can be explained by favourable effects on atrial transmural dispersion of refractoriness. 
While epicardial amiodarone adminstration in the present study produced a decrease in epicardial and endocardial atrial conduction velocities during sinus rhythm and during regular pacing, activation of the endocardium and epicardium remained similar, regarding both activation time and conduction direction. Simultaneously recorded epi-endocardial high-density electrogram maps during AF could not be analyzed properly due to severe fractionation, particularly in the epicardial electrograms. This may have been caused by the epicardially applied hydrogel, although fractionation was also observed in an experiment in which no hydrogel was applied (data not shown). In a previous study, intrapericardial delivery of sotalol and flecainide produced marked prolongation of epicardial AERP, whereas endocardial values were unchanged. ${ }^{15}$ However, no differences in epicardial versus endocardial AF cycle length prolongation were observed. It was therefore suggested that AF is dominated by the mid- and endocardial layers of the atria. This hypothesis was recently rejected, because simultaneous epi-endocardial mapping in goats with up to 6 months of sustained AF showed mean endo-epicardial activation time differences close to $0 \mathrm{~ms}$, arguing against preferential conduction from the epicardial layer to the endocardial bundles network or vice versa. ${ }^{20}$ In addition, simultaneous mapping during sustained AF did not show different activation rates in the endocardium versus epicardium, but a comparable AF cycle length in both layers of the atrial wall. Therefore, predominant pharmacological "elimination" of the epicardium through epicardial drug delivery may not necessarily lead to a more disorganized type of AF. However, we believe that achieving similar electrophysiological effects at both the epicardium and endocardium is a prerequisite for efficacious epicardial pharmacotherapy of AF. This notion is supported by the results of the present study, which suggests efficacy of epicardial amiodarone delivery in a model of sustained AF.

\section{Limitations}

An important consideration is whether results of these experiments can be extrapolated to the clinical setting, since the atrial structure and electrophysiology of patients with cardiovascular disease differs from electrically remodeled atria of otherwise-healthy goats. For example, in conscious goats that had no previous atrial disease and were submitted to electrically induced AF, the atrial refractory periodrate adaptation curve inverted, whereas in human atria inversion of the refractory period-cycle length curves were not observed. ${ }^{27}$ This may be explained by the fact that goats have a less steep increase in the refractory period toward long cycle lengths compared with other animals and patients.

A second limitation is that after epicardial application of hydrogel, reduced electrogram signal quality was observed, which hampered analysis of AF-mapping 
data. Whether the decrease in signal quality was a direct result of the hydrogel itself, or whether the reduced quality was caused by atrial tissue damage due to other experimental factors, is unclear. In addition, epicardial AERP measurements with the mapping tool were hindered after hydrogel application, rendering analysis of site-specific transmural dispersion of refractoriness nearly impossible. Future studies may circumvent this problem by epicardial application of amiodarone-eluting patches, which can be removed from the atria temporarily to allow higher electrogram quality. ${ }^{17}$

Finally, due to the small group size in this pilot study, statistical differences between groups could not be calculated. Larger studies are required to confirm results of the present study.

\section{Conclusions}

Epicardial delivery of amiodarone via application of a hydrogel release system produces an increase in AERP, while decreasing atrial conduction velocities, both at the atrial epicardium and endocardium. In contrast to previous studies that did not demonstrate efficacy of local antiarrhythmic pharmacotherapy in models of sustained AF, results of the present work suggest that epicardial amiodarone administration may reduce AF stability. It remains to be determined whether effects of epicardially delivered amiodarone on atrial transmural electrophysiology are unique among other antiarrhythmic drugs. 


\section{REFERENCES}

1. Fuster V, Ryden LE, Cannom DS, et al. ACC/AHA/ESC 2006 Guidelines for the Management of Patients with Atrial Fibrillation: a report of the American College of Cardiology/American Heart Association Task Force on Practice Guidelines and the European Society of Cardiology Committee for Practice Guidelines (Writing Committee to Revise the 2001 Guidelines for the Management of Patients With Atrial Fibrillation): developed in collaboration with the European Heart Rhythm Association and the Heart Rhythm Society. Circulation. 2006;114(7):e257354.

2. Camm AJ, Kirchhof P, Lip GY, et al. Guidelines for the management of atrial fibrillation: the Task Force for the Management of Atrial Fibrillation of the European Society of Cardiology (ESC). Eur Heart J. 2010;31(19):2369-2429.

3. Fuster V, Ryden LE, Cannom DS, et al. ACCF/AHA/HRS 2011 focused updates incorporated into the ACC/AHA/ESC 2006 guidelines for the management of patients with atrial fibrillation: a report of the American College of Cardiology Foundation/American Heart Association Task Force on practice guidelines. Circulation. 2011;123(10):e269-367.

4. Sintov A, Scott WA, Siden R, et al. Efficacy of epicardial controlled-release lidocaine for ventricular tachycardia induced by rapid ventricular pacing in dogs. J Cardiovasc Pharmacol. 1990;16(5):812-817.

5. Siden R, Kadish A, Flowers W, et al. Epicardial controlled-release verapamil prevents ventricular tachycardia episodes induced by acute ischemia in a canine model. J Cardiovasc Pharmacol. 1992;19(5):798-809.

6. Avitall B, Hare J, Zander G, et al. Iontophoretic transmyocardial drug delivery. A novel approach to antiarrhythmic drug therapy. Circulation. 1992;85(4):1582-1593.

7. Labhasetwar V, Underwood T, Gallagher M, et al. Sotalol controlled-release systems for arrhythmias: in vitro characterization, in vivo drug disposition, and electrophysiologic effects. $J$ Pharm Sci. 1994;83(2):156-164.

8. Ayers GM, Rho TH, Ben-David J, et al. Amiodarone instilled into the canine pericardial sac migrates transmurally to produce electrophysiologic effects and suppress atrial fibrillation. $J$ Cardiovasc Electrophysiol. 1996;7(8):713-721.

9. Fei L, Baron AD, Henry DP, et al. Intrapericardial delivery of L-arginine reduces the increased severity of ventricular arrhythmias during sympathetic stimulation in dogs with acute coronary occlusion: nitric oxide modulates sympathetic effects on ventricular electrophysiological properties. Circulation. 1997;96(11):4044-4049.

10. Darsinos JT, Karli JN, Samouilidou EC, et al. Distribution of amiodarone in heart tissues following intrapericardial administration. Int J Clin Pharmacol Ther. 1999;37(6):301-306.

11. Ujhelyi MR, Hadsall KZ, Euler DE, et al. Intrapericardial therapeutics: a pharmacodynamic and pharmacokinetic comparison between pericardial and intravenous procainamide delivery. J Cardiovasc Electrophysiol. 2002;13(6):605-611.

12. Kumar K, Nguyen $K$, Waxman $S$, et al. Potent antifibrillatory effects of intrapericardial nitroglycerin in the ischemic porcine heart. J Am Coll Cardiol. 2003;41(10):1831-1837.

13. Vereckei A, Gorski JC, Ujhelyi M, et al. Intrapericardial ibutilide administration fails to terminate pacing-induced sustained atrial fibrillation in dogs. Cardiovasc Drugs Ther. 2004;18(4):269277.

14. Kolettis TM, Kazakos N, Katsouras CS, et al. Intrapericardial drug delivery: pharmacologic properties and long-term safety in swine. Int J Cardiol. 2005;99(3):415-421. 
15. Van Brakel TJ, Hermans JJ, Accord RE, et al. Effects of intrapericardial sotalol and flecainide on transmural atrial electrophysiology and atrial fibrillation. J Cardiovasc Electrophysiol. 2009;20(2):207-215.

16. Bolderman RW, Hermans JJ, Rademakers LM, et al. Intrapericardial delivery of amiodarone and sotalol: atrial transmural drug distribution and electrophysiological effects. J Cardiovasc Pharmacol. 2009;54(4):355-363.

17. Bolderman RW, Bruin $P$, Hermans JJ, et al. Atrium-targeted drug delivery through an amiodarone-eluting bilayered patch. J Thorac Cardiovasc Surg. 2010;140(4):904-910.

18. Bolderman RW, Hermans JJ, Rademakers LM, et al. Epicardial application of an amiodaronereleasing hydrogel to suppress atrial tachyarrhythmias. Int J Cardiol. 2011;149(3):341-346.

19. Wijffels MC, Kirchhof CJ, Dorland R, et al. Atrial fibrillation begets atrial fibrillation. A study in awake chronically instrumented goats. Circulation. 1995;92(7):1954-1968.

20. Eckstein J, Maesen B, Linz D, et al. Time course and mechanisms of endo-epicardial electrical dissociation during atrial fibrillation in the goat. Cardiovasc Res. 2011;89(4):816-824.

21. Duytschaever $M$, Mast F, Killian $M$, et al. Methods for determining the refractory period and excitable gap during persistent atrial fibrillation in the goat. Circulation. 2001;104(8):957-962.

22. Benjamin EJ, Levy D, Vaziri SM, et al. Independent risk factors for atrial fibrillation in a population-based cohort. The Framingham Heart Study. JAMA. 1994;271(11):840-844.

23. Feng J, Yue $\mathrm{L}$, Wang $\mathrm{Z}$, et al. Ionic mechanisms of regional action potential heterogeneity in the canine right atrium. Circ Res. 1998;83(5):541-551.

24. Anyukhovsky EP, Rosenshtraukh LV. Electrophysiological responses of canine atrial endocardium and epicardium to acetylcholine and 4-aminopyridine. Cardiovasc Res. 1999;43(2):364-370.

25. Burashnikov A, Mannava S, Antzelevitch C. Transmembrane action potential heterogeneity in the canine isolated arterially perfused right atrium: effect of $I_{\mathrm{Kr}}$ and $I_{\mathrm{Kur}} / I_{\mathrm{to}}$ block. Am J Physiol Heart Circ Physiol. 2004;286(6):H2393-2400.

26. Antzelevitch C. Heterogeneity and cardiac arrhythmias: an overview. Heart Rhythm. 2007;4(7):964-972.

27. Franz MR, Karasik PL, Li C, et al. Electrical remodeling of the human atrium: similar effects in patients with chronic atrial fibrillation and atrial flutter. J Am Coll Cardiol. 1997;30(7):1785-1792. 


\section{CHAPTER 7}

General Discussion 


\section{INTRODUCTION}

Atrial fibrillation (AF) is an important clinical entity because of its high incidence, and its association with increased morbidity and mortality. ${ }^{1}$ The same holds true for AF occurring in the postoperative period of cardiac surgery. ${ }^{2}$ Although promising nonpharmacological therapies, particularly ablation procedures, have been developed, pharmacotherapy still remains the cornerstone of AF treatment. Pharmacotherapeutic management principles involve prevention of thromboembolism, and either a rate or rhythm control strategy. ${ }^{3}$ The choice between the latter two strategies has been the subject of extensive debate, which is in part due to the moderate effectiveness and proarrhythmic characteristics of contemporarily available drugs for rhythm control. Several new antiarrhythmic agents are currently evaluated in clinical studies, including compounds with multi-ion-channel-blocking properties, and compounds that selectively block atrium-specific currents. ${ }^{4}$ At the present time, the most frequently applied drug worldwide for maintaining sinus rhythm in patients with AF, is amiodarone; a highly lipophilic, multi-ion-channel-blocker. ${ }^{5}$ In comparison to other contemporary clinically-used antiarrhythmic agents, it combines high effectiveness with a low risk of torsades de pointes. However, major drawbacks of amiodarone involve its multiple systemic side effects, including thyroid, pulmonary, hepatic and dermatologic toxicity, as well as its complex and inconvenient pharmacokinetic profile (including slow and quite extensive tissue accumulation and very long half-life).

In order to improve the effectiveness of drugs and minimize their systemic toxicity, an alternative approach to development of new therapeutic agents may be adopted, namely local drug delivery. The heart is surrounded by the pericardial cavity, which is ideally suited for local cardiac pharmacotherapy, because it provides a natural drug reservoir and space for epicardial application of drug delivery systems. In the last decade, minimally-invasive techniques to access the noneffusive pericardial cavity have been optimized. ${ }^{6}$ Whereas, historically, intrapericardial (IPC) drug delivery has been used primarily for treatment of pericarditis, present advances in the field of pericardial access and drug delivery systems hold out the prospect of treating arrhythmias, coronary artery disease and myocardial disease.

A number of studies have investigated IPC delivery of antiarrhythmic agents, including lidocaine, digoxin, amiodarone, beta blockers, procainamide, ibutilide, sotalol, and flecainide..$^{7-15}$ Pharmacokinetic advantages and significant effects on atrial electrophysiological parameters were invariably demonstrated. However, IPC delivery of ibutilide, sotalol, and flecainide did not result in higher AF conversion rates compared to controls in animal models of persistent AF. ${ }^{13,15}$ This was hypothesized to be partly due to the attenuated effects of class III antiarrhythmic agents in 
chronically remodeled atria, and also to possibly insufficient drug loading of the endocardial areas. ${ }^{15}$ Indeed, steep transmural myocardial concentrations of both sotalol and flecainide were observed in atria and ventricles. In addition, instillation of flecainide in the pericardial cavity produced marked ST-elevations. ${ }^{15}$ This suggests that IPC drug delivery of antiarrhythmic drugs, via resultant transmural concentration gradients, may promote electrophysiological dispersion between epicardial and endocardial layers, which in itself might be proarrhythmic.

The first objective of the present thesis was to investigate electrophysiological effects during epicardial amiodarone therapy. The hypothesis tested was that epicardial delivery of this highly lipophilic, multi-ion-channel-blocker would attenuate inducibility of atrial arrhythmias, despite the presence of a transmural concentration gradient. Second, the current thesis explored three approaches for epicardial amiodarone delivery, namely IPC-infused amiodarone solutions, epicardially sprayed in-situ produced hydrogels, and epicardially sutured bilayered patches. The latter two drug delivery systems are composed of synthetic biodegradable polymers. It was evaluated whether these locally-applied biomaterials would enable long-term, atrium-specific amiodarone release, while maintaining low systemic drug levels.

\section{MAIN FINDINGS}

\section{Short-Term Intrapericardial Amiodarone Delivery}

To accurately study amiodarone distribution after IPC delivery, a novel method was developed for determination of amiodarone concentrations in plasma, pericardial fluid, and tissue samples (see Chapter 2). This technique required only small sample-quantities, and showed linear curves in a wide range of amiodarone concentrations, which enabled measurement of the transmural concentration gradients that are typical for epicardial drug delivery. In addition, this method was optimized for detection of dronedarone, a noniodinated benzofuran derivative envisioned as a safer alternative to amiodarone. ${ }^{16}$

A previous study reported that IPC-delivered amiodarone migrates transmurally and suppresses electrically-induced AF. ${ }^{8}$ However, only mean atrial drug concentrations and only epicardial electrophysiological effects were measured. More recent work showed steep transmural drug concentrations in atria after IPC administration of sotalol, while AF conversion rates were not different to IV infusion. ${ }^{15}$ Therefore, a series of acute experiments was performed in which atrial transmural drug distribution and electrophysiological effects of IPC amiodarone were compared with IPC sotalol (see Chapter 3). Remarkably, despite distinct differences in 
the physicochemical properties of both drugs in terms of molecular weight, hydrophilicity and electrical charge, IPC delivery produced similar drug distribution patterns across both atrial walls. Regarding atrial transmural electrophysiology, however, IPC amiodarone and sotalol exerted dissimilar effects. In contrast with sotalol, instillation of amiodarone into the pericardial cavity produced a relatively small increase in epicardial atrial effective refractory period, while inducibility of atrial arrhythmias was significantly reduced, independent of the site of burst-pacing (endocardially and epicardially). One possible explanation for these findings could be that maximal electrophysiological effects of acute amiodarone may already be achieved at relatively low myocardial concentrations. Another contributing factor for the inconsistent relationship between transmural amiodarone concentrations and effects on epicardial and endocardial electrophysiology might be transmural heterogeneity in ionic currents, which has been demonstrated to exist also in the thin atria. ${ }^{17-20}$

\section{Long-Term Epicardial Amiodarone Delivery}

While IPC infusion of amiodarone solutions was only used to evaluate short-term effects of epicardial delivery, the feasibility of chronic local amiodarone therapy was investigated by means of epicardial application of synthetic biodegradable polymers. In a first series of experiments in goats, an in-situ produced poly(ethylene glycol)-based hydrogel was loaded with amiodarone (see Chapter 4). This twocomponent tissue sealant, which is proven effective in prevention of pericardial adhesions after cardiac surgery, ${ }^{21,22}$ was sprayed onto the atrial epicardium. The right atrium was chosen as delivery site, in order to determine the negative chronotropic effects of local amiodarone application near the sinoatrial node. Subsequent concentration measurements showed therapeutic amiodarone levels in the right atrial epicardium up to several weeks after hydrogel application, whereas ventricular and extracardiac amiodarone tissue concentrations remained 1-2 orders of magnitude lower. However, epicardial concentrations amiodarone in the left atrium and ventricles were both higher than endocardial levels in the right atrium. This is most likely explained by a relatively faster epicardial (longitudinal) than transmural (transversal) distribution, because the former is facilitated by simultaneous drug uptake and release via the pericardial fluid. ${ }^{11}$

In a second series of experiments, bilayered patches with a poly(ethylene glycol)-based drug-loaded matrix and poly(lactide-co-caprolactone)-based backing layer were sutured to the right atrial epicardium (see Chapter 5). The rationale of this approach, is that the backing layer minimizes drug leakage to the pericardial fluid. This reduces distribution to the ventricular epicardium and the parietal pericardium, and, therefore, diminishes redistribution to extracardiac tissues. Indeed, in 
contrast to hydrogel application, bilayered patches produced very high amiodarone concentrations in the right atrium compared with other heart chambers, both in the epicardial and endocardial layers. Ventricular and extracardiac amiodarone levels were 2-4 orders of magnitude lower throughout the follow-up period. Hydrogel and patch matrices yielded monoexponentially decreasing amiodarone concentrations, which was probably both diffusion and degradation mediated, because poly(ethylene glycol)-based polymers with thioester bonds degrade within several weeks. ${ }^{21}$ However, the rate of decline in right atrial drug levels was less after patch application, possibly due to shielding of the drug-loaded matrix by the poly(lactideco-caprolactone)-based backing layer. The latter copolymer degrades much slower, on the order of 6 to 12 months. ${ }^{23}$

Regarding electrophysiological effects of chronic epicardial amiodarone delivery, both hydrogel and patches produced increases in right epicardial AERPs and conduction times. In addition, the inducibility of atrial arrhythmia was suppressed in comparison with control groups. These effects lasted for three weeks in the hydrogel-treated animals, while amiodarone-loaded patches still exerted significant effects at the end of the experimental four-week period. Interestingly, AERPs were maximally prolonged in the first days after application, whereas conduction times further increased during chronic amiodarone delivery. The latter may be explained by treatment duration-dependent changes in amiodarone's ionic current blocking profile and by postoperative factors that affected conduction time. ${ }^{24}$ In the control groups, atrial vulnerability to tachyarrhythmias showed a peak on days 2 and 3 after thoracotomy, which coincides with the peak incidence of clinical postoperative AF. ${ }^{25}$ Because epicardial application of amiodarone-eluting polymers reduced peak occurence in both series of experiments, the effectivess of this approach in preventing postoperative AF is anticipated.

\section{The Effectiveness of Epicardial Amiodarone in Remodeled Atria}

To elucidate transmural electrophysiological effects of epicardial amiodarone delivery in remodeled atria, a novel high-density mapping tool was employed in a goatmodel of persistent AF (see Chapter 6). In contrast to previous studies that investigated AF conversion rates after IPC-delivered ibutilide, flecainide and sotalol,13,15 initial results of the present work suggest local amiodarone administration to be effective. Unfortunately, epicardial hydrogel application reduced electrogram signal quality, which hampered analyses of the AF-mapping data. In addition, the hydrogel hindered epicardial AERP measurements with the mapping tool, rendering analysis of site-specific transmural dispersion of refractoriness nearly impossible. Data acquired during sinus rhythm, and during 400ms and 250ms interval-pacing showed prolongation of both epicardial and endocardial AERPs and conduction 
times. However, the number of experiments, in which sufficient data could be collected, was too small to draw statistically significant conclusions. Therefore, further research is needed to assess effectiveness of epicardial amiodarone in remodeled atria.

\section{Critical Reflection}

The present thesis explored epicardial delivery of amiodarone in pre-clinical studies performed in goats. An important consideration is whether results of these experiments can be extrapolated to the clinical setting, since animal models never exactly replicate the clinical condition. Although they allow full access to tissues, highly invasive measurements and carefully controlled conditions, any animal model is an exploration of a mechanistic hypothesis that may or may not be applicable to human disease. ${ }^{26}$

The atrial structure and electrophysiology of patients with cardiovascular disease differs from the atrium of healthy goats. For example, in conscious goats that had no previous atrial disease and were submitted to electrically induced AF, the atrial refractory period-rate adaptation curve inverted, whereas in human atria inversion of the refractory period-cycle length curves were not observed. ${ }^{27}$ This may be explained by the fact that goats have a less steep increase in the refractory period toward long cycle lengths compared with other animals and patients. ${ }^{27}$ Furthermore, in clinical studies the pathological substrate of AF is affected by several factors, including underlying cardiac disease, comorbidity and concomitant drug therapy.

In the experiments presented in the current thesis, IPC amiodarone infusion and chronic local administration were performed in non-remodeled atria. Therefore, duration of pacing-induced arrhythmias was short, generally lasting several seconds, and sustained episodes of AF did not occur. Additional studies in representative animal models are required to evaluate the effectiveness of long-term local amiodarone delivery in the treatment of chronic AF. In this regard, the study described in Chapter 6 forms a prelude to further research on effectiveness of epicardial amiodarone in patients with chronic AF.

The present work does not provide a definite answer to the question as to what extent atrial and ventricular transmural electrophysiology are affected by epicardial delivery of amiodarone. This is because the most thorough method currently available, namely simultaneous high-density mapping of the epicardial and endocardial atrial surfaces, proved to be hampered by the drug-releasing polymer layers. The quadripolar catheters and patch electrodes, used in the IPC and chronic amiodarone 
delivery experiments, were not well suited for true site-specific simultaneous epicardial and endocardial recordings.

Another point of attention concerns safety of epicardial amiodarone delivery. Current studies did not specifically assess ventricular proarrhythmia, for example by measuring ventricular fibrillation thresholds. Also, histologic analysis was omitted in favor of using the atrial tissue for drug concentration measurements. Although no macroscopic adverse effects of chronic epicardial delivery were observed, local application of amiodarone and (degradation of) biomaterials might produce toxic effects, such as necrosis or inflammation.

\section{FUTURE DIRECTIONS}

Epicardial drug delivery has shown higher deposition and retention of therapeutic compounds than either intravenous, intracoronary, or intramyocardial delivery. ${ }^{28-30}$ In addition, epicardial drug delivery systems may serve as scaffolds for tissue engineering, as barriers for prevention of pericardial adhesions, or as coatings for pacing electrodes. ${ }^{22,30-33}$ In combination with recent advances in pericardial access techniques, ${ }^{6,34}$ this approach may become of increasing importance in the treatment of cardiac disease. Some of the numerous therapeutic applications of epicardial drug delivery have been described in previous sections of this thesis (see Chapter 1). The remainder of this section focuses on future perspectives of epicardial pharmacotherapy of atrial arrhythmias.

\section{Choice of Antiarrhythmic Agent}

The available evidence argues against epicardial delivery of pure class I and III antiarrhyhmic drugs, such as flecainide and ibutilide, for the treatment of atrial arrhythmias, because the resultant transmural concentration gradients may promote their proarrhythmic effect. ${ }^{15}$ Local administration of novel atrium-specific channel-blockers would likely abolish the risk of torsades des pointes. ${ }^{35}$ However, theoretically, atrial transmural concentrations gradients of these agents might increase inducibility of atrial arrhythmias. Myocardial gradients of multi-ion channelblockers, such as amiodarone, could result in less electrophysiological heterogeneity, although further studies are required to support this hypothesis. Promising new compounds with mult-ion channel-blocking properties include dronedarone, budiodarone, celivarone, vernakalant, and ranolazine. ${ }^{4}$ Interestingly, very recent data suggest that IPC-delivered ranolazine exhibits striking atrial antiarrhythmic actions with mild protective effects on ventricular fibrillation inducibility. ${ }^{36}$ Because the combination of ranolazine and chronic amiodarone produces potent synergistic antiarrhythmic effects, this might be a favourable duo for IPC administration. ${ }^{37}$ 
Another approach to circumvent epicardial delivery-induced electrophysiological dispersion, is to apply non-channel-blocking agents. Especially upstream therapies, which are believed to reduce atrial structural remodeling, may offer a relatively safe alternative to classic antiarrhythmic drugs. ${ }^{38}$ These therapies include inhibitors of the renin-angiotensin-aldosterone system and antioxidative agents. For prevention of post-operative AF, epicardial steroid application is of particular interest, because inflammation plays a significant role in its pathophysiology. ${ }^{39}$ Finally, complete transmural atrial gene transfer has been recently introduced in the field of epicardial delivery therapies. ${ }^{40}$ Ultimately, use of long-term expression vectors could cure or even prevent AF. ${ }^{41}$

\section{Advances in Drug Delivery Systems}

In contrast to chronic percutaneous IPC drug infusion, impantable (osmotic) minipumps and ionphoretic delivery systems, biodegradable drug delivery systems may pose less risk of infection, obstruction or mechanical failure. Biomaterials allow time-controlled delivery and stimulus-responsive release characteristics, such as thermosensitivity, inflammation-responsivess, and ultrasound-induced release. ${ }^{42,43}$ Although polymers capable of arrhythmia-responsive drug delivery have not yet been reported, development of these biomaterials might enable efficient treatment of paroxysmal arrhythmias, requiring lower total drug doses.

Various formulations for epicardial drug delivery have been investigated, including hydrogels, microspheres and patches. Each formulation has intrinsic advantages and disadvantages. For example, hydrogels and microspheres can be easily injected into the pericardial cavity, but these formulations are likely less-suitable for atrium-specific drug delivery. Patches may allow a more localized release of drugs, but patch-adhesion is hampered by cardiac contractions. Improvement in patchadhesion characteristics is desired for further clinical application.

Although epicardial delivery of antiarrhythmic drugs to target the atrial myocardium has been studied most frequently, the pulmonary veins and the cardiac nervous system, considering their important role in atrial arrhythmogenesis, ${ }^{44,45}$ are interesting alternative targets for local pharmacotherapy. However, the anatomy of these tissues requires development of tailored drug release systems, because of the delicate structure of the pulmonary veins, and the complex localization of the cardiac ganglia.

Perhaps the most extensive clinical experience with epicardial pharmacotherapy has been obtained with steroid-eluting pacing leads. ${ }^{33}$ These composite biomaterials are primarily used to reduce local inflammation and stimulation thresholds. In addition, electrode coatings might be loaded with antiarrhythmic drugs, non-channelblocking agents (upstream therapy), or gene vectors to employ pacing leads for 
local antiarrhythmic pharmacotherapy. ${ }^{46}$ In the postoperative setting, biodegradable matrices may be used to fix temporary epicardial pacing leads to the epicardium or to achieve low-energy internal cardioversion. ${ }^{47,48}$ Further studies are needed to investigate the clinical efficacy and safety of these devices.

\section{CONCLUSIONS}

Pharmacotherapy of cardiac disease is often accompagnied by systemic side effects. This also holds true for antiarrhythmic treatment of atrial fibrillation, the most common cardiac arrhythmia requiring medical attention. The present thesis demonstrates that local cardiac pharmacotherapy via intrapericardial and epicardial delivery of amiodarone may improve its effectiveness and minimize its systemic toxicity. Epicardial application of synthetic biodegradable polymers might enable long-term, atrium-specific amiodarone release. An obvious initial clinical application of epicardial amiodarone delivery systems would be prevention of postoperative atrial fibrillation. Further improvements in percutaneous pericardial access methods and drug delivery systems could allow epicardial pharmacotherapy of chronic cardiac disease. 


\section{REFERENCES}

1. Benjamin EJ, Levy D, Vaziri SM, et al. Independent risk factors for atrial fibrillation in a population-based cohort. The Framingham Heart Study. JAMA. 1994;271(11):840-844.

2. Hogue CW, Jr., Creswell LL, Gutterman DD, et al. Epidemiology, mechanisms, and risks: American College of Chest Physicians guidelines for the prevention and management of postoperative atrial fibrillation after cardiac surgery. Chest. 2005;128(2 Suppl):9S-16S.

3. Fuster V, Ryden LE, Cannom DS, et al. ACC/AHA/ESC 2006 Guidelines for the Management of Patients with Atrial Fibrillation: a report of the American College of Cardiology/American Heart Association Task Force on Practice Guidelines and the European Society of Cardiology Committee for Practice Guidelines (Writing Committee to Revise the 2001 Guidelines for the Management of Patients With Atrial Fibrillation): developed in collaboration with the European Heart Rhythm Association and the Heart Rhythm Society. Circulation. 2006;114(7):e257354.

4. Mason PK, DiMarco JP. New pharmacological agents for arrhythmias. Circ Arrhythm Electrophysiol. 2009;2(5):588-597.

5. Singh BN. Amiodarone as paradigm for developing new drugs for atrial fibrillation. J Cardiovasc Pharmacol. 2008;52(4):300-305.

6. Rupp H, Rupp TP, Alter P, et al. Intrapericardial procedures for cardiac regeneration by stem cells: need for minimal invasive access (AttachLifter) to the normal pericardial cavity. Herz. 2010;35(7):458-465.

7. Darsinos JT, Samouilidou EC, Krumholz B, et al. Distribution of lidocaine and digoxin in heart tissues and aorta following intrapericardial administration. Int J Clin Pharmacol Ther Toxicol. 1993;31(12):611-615.

8. Ayers GM, Rho TH, Ben-David J, et al. Amiodarone instilled into the canine pericardial sac migrates transmurally to produce electrophysiologic effects and suppress atrial fibrillation. $J$ Cardiovasc Electrophysiol. 1996;7(8):713-721.

9. Darsinos JT, Karli JN, Samouilidou EC, et al. Distribution of amiodarone in heart tissues following intrapericardial administration. Int J Clin Pharmacol Ther. 1999;37(6):301-306.

10. Moreno $R$, Waxman $S$, Rowe $K$, et al. Intrapericardial beta-adrenergic blockade with esmolol exerts a potent antitachycardic effect without depressing contractility. J Cardiovasc Pharmacol. 2000;36(6):722-727.

11. Ujhelyi MR, Hadsall KZ, Euler DE, et al. Intrapericardial therapeutics: a pharmacodynamic and pharmacokinetic comparison between pericardial and intravenous procainamide delivery. J Cardiovasc Electrophysiol. 2002;13(6):605-611.

12. Van Brakel TJ, Hermans JJ, Janssen BJ, et al. Intrapericardial delivery enhances cardiac effects of sotalol and atenolol. J Cardiovasc Pharmacol. 2004;44(1):50-56.

13. Vereckei A, Gorski JC, Ujhelyi M, et al. Intrapericardial ibutilide administration fails to terminate pacing-induced sustained atrial fibrillation in dogs. Cardiovasc Drugs Ther. 2004;18(4):269277.

14. Kolettis TM, Kazakos N, Katsouras CS, et al. Intrapericardial drug delivery: pharmacologic properties and long-term safety in swine. Int J Cardiol. 2005;99(3):415-421.

15. Van Brakel TJ, Hermans JJ, Accord RE, et al. Effects of intrapericardial sotalol and flecainide on transmural atrial electrophysiology and atrial fibrillation. $J$ Cardiovasc Electrophysiol. 2009;20(2):207-215.

16. Hohnloser SH, Crijns HJ, van Eickels $\mathrm{M}$, et al. Effect of dronedarone on cardiovascular events in atrial fibrillation. N Engl J Med. 2009;360(7):668-678. 
17. Feng J, Yue L, Wang $\mathrm{Z}$, et al. Ionic mechanisms of regional action potential heterogeneity in the canine right atrium. Circ Res. 1998;83(5):541-551.

18. Anyukhovsky EP, Rosenshtraukh LV. Electrophysiological responses of canine atrial endocardium and epicardium to acetylcholine and 4-aminopyridine. Cardiovasc Res. 1999;43(2):364-370.

19. Burashnikov A, Mannava S, Antzelevitch C. Transmembrane action potential heterogeneity in the canine isolated arterially perfused right atrium: effect of $\mathrm{IKr}$ and IKur/Ito block. Am J Physiol Heart Circ Physiol. 2004;286(6):H2393-2400.

20. Antzelevitch C. Role of spatial dispersion of repolarization in inherited and acquired sudden cardiac death syndromes. Am J Physiol Heart Circ Physiol. 2007;293(4):H2024-2038.

21. Hendrikx M, Mees U, Hill AC, et al. Evaluation of a novel synthetic sealant for inhibition of cardiac adhesions and clinical experience in cardiac surgery procedures. Heart Surg Forum. 2001;4(3):204-210.

22. Konertz WF, Kostelka M, Mohr FW, et al. Reducing the incidence and severity of pericardial adhesions with a sprayable polymeric matrix. Ann Thorac Surg. 2003;76(4):1270-1274.

23. Pitt CG, Gratzl MM, Kimmel GL, et al. Aliphatic polyesters II. The degradation of poly (DLlactide), poly (epsilon-caprolactone), and their copolymers in vivo. Biomaterials. 1981;2(4):215220.

24. Kodama I, Kamiya K, Toyama J. Amiodarone: ionic and cellular mechanisms of action of the most promising class III agent. Am J Cardiol. 1999;84(9A):20R-28R.

25. Mathew JP, Fontes ML, Tudor IC, et al. A multicenter risk index for atrial fibrillation after cardiac surgery. JAMA. 2004;291(14):1720-1729.

26. Nattel S, Shiroshita-Takeshita A, Brundel BJ, et al. Mechanisms of atrial fibrillation: lessons from animal models. Prog Cardiovasc Dis. 2005;48(1):9-28.

27. Franz MR, Karasik PL, Li C, et al. Electrical remodeling of the human atrium: similar effects in patients with chronic atrial fibrillation and atrial flutter. J Am Coll Cardiol. 1997;30(7):1785-1792.

28. Lazarous DF, Shou M, Stiber JA, et al. Pharmacodynamics of basic fibroblast growth factor: route of administration determines myocardial and systemic distribution. Cardiovasc Res. 1997;36(1):78-85.

29. Lamping KG, Rios CD, Chun JA, et al. Intrapericardial administration of adenovirus for gene transfer. Am J Physiol. 1997;272(1 Pt 2):H310-317.

30. Hamdi H, Furuta A, Bellamy V, et al. Cell delivery: intramyocardial injections or epicardial deposition? A head-to-head comparison. Ann Thorac Surg. 2009;87(4):1196-1203.

31. Kellar RS, Landeen LK, Shepherd BR, et al. Scaffold-based three-dimensional human fibroblast culture provides a structural matrix that supports angiogenesis in infarcted heart tissue. Circulation. 2001;104(17):2063-2068.

32. Kutschka I, Chen IY, Kofidis T, et al. Collagen matrices enhance survival of transplanted cardiomyoblasts and contribute to functional improvement of ischemic rat hearts. Circulation. 2006;114(1 Suppl):I167-173.

33. Tomaske M, Gerritse B, Kretzers L, et al. A 12-year experience of bipolar steroid-eluting epicardial pacing leads in children. Ann Thorac Surg. 2008;85(5):1704-1711.

34. Waxman S, Pulerwitz TC, Rowe KA, et al. Preclinical safety testing of percutaneous transatrial access to the normal pericardial space for local cardiac drug delivery and diagnostic sampling. Catheter Cardiovasc Interv. 2000;49(4):472-477.

35. Ehrlich JR, Nattel S. Atrial-selective pharmacological therapy for atrial fibrillation: hype or hope? Curr Opin Cardiol. 2009;24(1):50-55. 
36. Carvas M, Nascimento BC, Acar M, et al. Intrapericardial ranolazine prolongs atrial refractory period and markedly reduces atrial fibrillation inducibility in the intact porcine heart. J Cardiovasc Pharmacol. 2010;55(3):286-291.

37. Sicouri S, Burashnikov A, Belardinelli L, et al. Synergistic electrophysiologic and antiarrhythmic effects of the combination of ranolazine and chronic amiodarone in canine atria. Circ Arrhythm Electrophysiol. 2010;3(1):88-95.

38. Smit MD, Van Gelder IC. Upstream therapy of atrial fibrillation. Expert Rev Cardiovasc Ther. 2009;7(7):763-778.

39. Davis EM, Packard KA, Hilleman DE. Pharmacologic prophylaxis of postoperative atrial fibrillation in patients undergoing cardiac surgery: beyond beta-blockers. Pharmacotherapy. 2010;30(7):749, 274e-318e.

40. Kikuchi K, McDonald AD, Sasano T, et al. Targeted modification of atrial electrophysiology by homogeneous transmural atrial gene transfer. Circulation. 2005;111(3):264-270.

41. Amit G, Kikuchi K, Greener ID, et al. Selective molecular potassium channel blockade prevents atrial fibrillation. Circulation. 2010;121(21):2263-2270.

42. Kikuchi A, Okano T. Pulsatile drug release control using hydrogels. Adv Drug Deliv Rev. 2002;54(1):53-77.

43. Norris P, Noble M, Francolini I, et al. Ultrasonically Controlled Release of Ciprofloxacin from Self-Assembled Coatings on Poly(2-Hydroxyethyl Methacrylate) Hydrogels for Pseudomonas aeruginosa Biofilm Prevention. Antimicrob Agents Chemother. 2005;49(10):4272-4279.

44. Haissaguerre M, Jais $\mathrm{P}$, Shah DC, et al. Spontaneous initiation of atrial fibrillation by ectopic beats originating in the pulmonary veins. N Engl J Med. 1998;339(10):659-666.

45. Volders PG. Viewpoint series: Novel insights into the role of the sympathetic nervous system in cardiac arrhythmogenesis. Heart Rhythm. 2010;7(12):1900-6.

46. Labhasetwar V, Strickberger SA, Underwood T, et al. Prevention of acute inducible atrial flutter in dogs by using an ibutilide-polymer-coated pacing electrode. J Cardiovasc Pharmacol. 1998;31(3):449-455.

47. Narita Y, Fukuhira Y, Kagami H, et al. Development of a novel temporary epicardial pacing wire with biodegradable film. Ann Thorac Surg. 2006;82(4):1489-1493.

48. Iino K, Yui N, Ooya T, et al. Successful low-energy cardioversion using a novel biodegradable gel pad: Feasibility of treating postoperative atrial fibrillation in animals. J Thorac Cardiovasc Surg. 2007;134(6):1519-1525. 
SUMMARY 
Atrial fibrillation is an important clinical entity because of its high incidence, and its association with increased morbidity and mortality. Although promising nonpharmacological therapies, particularly ablation procedures, have been developed, pharmacotherapy still remains the cornerstone of AF treatment. However, currently available antiarrhythmic drugs are only moderately effective and all carry risks of serious side effects, most notably ventricular arrhythmias.

Amiodarone, a highly lipophilic multi-ion channel-blocking antiarrhythmic compound, is the most frequently used agent worldwide for maintaining sinus rhythm in patients with AF. It displays superior efficacy combined with a low potential for causing torsades de pointes. Unfortunately, amiodarone therapy is also associated with numerous systemic side effects, including thyroid, pulmonary, hepatic and dermatologic toxicity.

In order to improve drug efficacy and minimize systemic toxicity, an alternative approach to development of new therapeutic agents may be adopted, namely local drug delivery. The heart is surrounded by the pericardial cavity, which is ideally suited for local cardiac pharmacotherapy, because it provides a natural drug reservoir and space for epicardial application of drug delivery systems. The present thesis explores electrophysiological effects and drug distribution during intrapericardial and epicardial delivery of amiodarone.

Chapter 1 reviews electrophysiological mechanisms and clinical aspects of atrial fibrillation and postoperative atrial fibrillation. A second section of this chapter focuses on pharmacokinetics and electrophysiological effects of amiodarone. The chapter ends with a review on intrapericardial and epicardial drug delivery.

Chapter 2 describes a novel method for the determination of amiodarone and dronedarone, and their principal metabolites in plasma and myocardium by highperformance liquid chromatography and UV-detection. Dronedarone, a noniodinated benzofuran derivative of amiodarone, has recently been regulatory approved for maintenance of sinus rhythm in patients with atrial fibrillation. Plasma and myocardial sample preparation included deproteinization with acetonitrile and extraction with a mixture of heptane and dichloromethane, followed by chromatographic separation on a polymeric C18 column. The proposed HPLC-UV method was validated with respect to linearity, accuracy, precision, recovery, limit of detection and lower limit of quantification.

Chapter 3 compares atrial transmural drug distribution and electrophysiological effects during intrapericardial and intravenous delivery of amiodarone and sotalol in goats. Despite distinct differences in physicochemical properties of both drugs in terms of molecular weight, hydrophilicity and electrical charge, intrapericardial delivery produced similar distributions across both atrial walls. Regarding atrial transmural electrophysiology, however, intrapericardial amiodarone and sotalol exerted dissimilar effects. In contrast with sotalol, instillation of amiodarone into the 
pericardial cavity produced a relatively small increase in epicardial atrial effective refractory period, while inducibility of atrial arrhythmias was significantly reduced, independent of the site of burst-pacing (endocardially and epicardially). Plasma drug concentrations were significantly lower during intrapericardial delivery compared with intravenous infusion.

Chapter 4 explores feasibility of prolonged and localized epicardial amiodarone-release via an in-situ forming biodegradable poly(ethylene glycol)-based hydrogel, that is already used for prevention of pericardial adhesions after cardiac surgery. In experiments with goats the two-component polymer system was sprayed onto the epicardial surface of the right atrium. Subsequent concentration measurements showed therapeutic amiodarone levels in the right atrial epicardium up to several weeks after hydrogel application, whereas ventricular and extracardiac amiodarone tissue concentrations remained 1-2 orders of magnitude lower. However, epicardial concentrations amiodarone in the left atrium and ventricles were both higher than endocardial levels in the right atrium, probably due to relatively faster epicardial than transmural distribution, facilitated by simultaneous drug uptake and release via the pericardial fluid.

Chapter 5 addresses the question whether more atrium-specific drug delivery could be achieved through application of bilayered patches with a poly(ethylene glycol)-based drug-loaded matrix and poly(lactide-co-caprolactone)-based backing layer. The rationale of this approach, is that the backing layer minimizes drug leakage to the pericardial fluid. Bilayered patches produced very high amiodarone concentrations in the right atrium, whereas ventricular and extracardiac amiodarone levels were 2-4 orders of magnitude lower throughout the follow-up period.

Both hydrogel and patches produced increases in right epicardial atrial effective refractory periods and conduction times. In addition, inducibility of atrial arrhythmia was suppressed in comparison with control groups. These effects lasted for three weeks in the hydrogel-treated animals, while amiodarone-loaded patches still exerted significant effects at the end of the experimental four-week period.

Chapter 6 focuses on epicardial delivery of amiodarone in a goat-model of burst-pacing-induced persistent atrial fibrillation. A novel mapping tool was employed for simultaneous epicardial and endocardial high-density mapping in order to evaluate the effects of transmural myocardial amiodarone gradients on electrophysiological parameters. Initial results suggest efficacy of local amiodarone administration, but epicardial hydrogel application hindered epicardial refractoriness measurements and signal quality during atrial fibrillation. Further research is needed to elucidate electrophysiological effects of epicardial amiodarone in remodeled atria. 
Chapter 7 discusses the main findings of this thesis, integrates these into a broader scientific perspective, and touches upon possible implications for future research and clinical management.

\section{Conclusions}

The present thesis demonstrates that local cardiac pharmacotherapy through intrapericardial and epicardial delivery of amiodarone may improve efficacy and minimize systemic toxicity. Epicardial application of synthetic biodegradable polymers might enable long-term, atrium-specific amiodarone release. These drug delivery systems could be used clinically to prevent postoperative atrial fibrillation. Further refinement of access methods and release systems may allow epicardial pharmacotherapy of chronic cardiac disease. 
SAMENVATTING 
Atriumfibrilleren is een belangrijke medische aandoening omdat het een hoge incidentie heeft en gepaard gaat met een hogere morbiditeit en mortaliteit. Hoewel er reeds veelbelovende niet-farmacologische therapieën (in het bijzonder de ablatie procedures) ontwikkeld zijn, vormt farmacotherapie nog steeds de hoeksteen van de behandeling van atriumfibrilleren.

Amiodaron, een zeer lipofiele stof die meerdere ionkanalen blokkeert, is wereldwijd het meest gebruikte geneesmiddel om sinusritme te bevorderen in patiënten met atriumfibrilleren. Deze stof combineert een superieure effectiviteit met een laag risico op ventriculaire bijwerkingen (torsades de pointes). Jammergenoeg gaat behandeling met amiodaron gepaard met een breed scala aan systemische bijwerkingen, zoals schildklier-, long-, lever- en huidtoxiciteit.

Om de effectiviteit te verbeteren en om systemische toxiciteit te minimaliseren, kan voor een andere benadering gekozen worden, namelijk lokale toediening van geneesmiddelen. Het hart wordt omgeven door de pericardiale holte, die zeer geschikt is voor lokale cardiale toediening van geneesmiddelen, omdat deze een natuurlijk reservoir vormt en ruimte biedt voor epicardiale aanbrenging van geneesmiddelen toedieningssystemen. Dit proefschrift onderzoekt de elektrofysiologische effecten en medicijndistributie tijdens intrapericardiale en epicardiale toediening van amiodaron.

Hoofdstuk 1 biedt een literatuuroverzicht van elektrofysiologische mechanismen en klinische aspecten van atriumfibrilleren en postoperatief atriumfibrilleren. Een tweede sectie van dit hoofdstuk richt zich op de farmacokinetiek en elektrofysiologische effecten van amiodaron. Het hoofdstuk eindigt met een literatuuroverzicht over intrapericardiale en epicardiale toediening van geneesmiddelen.

Hoofdstuk 2 beschrijft een nieuwe methode om concentraties van amiodaron, dronedaron en hun voornaamste metabolieten te bepalen in plasma en myocardweefsel met behulp van vloeistofchromatografie en UV-detectie. Dronedaron, een niet-gejodeerd benzofuraan derivaat van amiodaron, is recent geregistreerd voor behoud van sinusritme in patienten met boezemfibrilleren. Plasma- en myocardmonsters werden eerst bewerkt door middel van proteolyse met acetonitril en extractie met een mengsel van heptaan en dichloormethaan, gevolgd door chromatografische scheiding op een polymere C18 kolom. De methode werd gevalideerd met betrekking tot lineariteit, accuratesse, precisie, recuperatie, detectielimiet en minimale kwantificatielimiet.

Hoofdstuk 3 vergelijkt atriale transmurale medicijndistributie en elektrofysiologische effecten tijdens intrapericardiale en intraveneuze toediening van amiodaron en sotalol in geiten. Ondanks uitgesproken verschillen in fysicochemische eigenschappen van beide medicijnen, onder andere in molecuulmassa, hydrofiliciteit, en elektrische lading, resulteerde intrapericardiale toediening in gelijkaardige distributie over beide atriumwanden. Wat betreft atriale transmurale elektrofysiologie, 
veroorzaakten intrapericardiaal amiodaron en sotalol ongelijke effecten. In tegenstelling tot sotalol, produceerde infusie van amiodaronoplossingen in de pericardholte een relatief kleine toename van de atriale effectieve refractaire periode, terwijl de induceerbaarheid van atriale aritmieën significant afnam, onafhankelijk van de locatie van burst-pacing (endocardiaal of epicardiaal). Plasma medicijnconcentraties waren significant lager tijdens intrapericardiale toediening vergeleken met intraveneuze infusie.

Hoofdstuk 4 onderzoekt de haalbaarheid van verlengde en gelokaliseerde epicardiale amiodaron-vrijzetting via een in-situ vormende, biodegradabele, op polyethyleenglycol-gebaseerde hydrogel, die reeds gebruikt wordt ter preventie van postoperatieve pericardiale adhesies. Tijdens experimenten met geiten werd het twee-componenten, polymere geneesmiddelen toedieningssysteem op het epicardiale oppervlak van rechter atria gespoten. Daaropvolgende concentratiebepalingen toonden therapeutische amiodaronspiegels in het epicard van het rechter atrium gedurende enkele weken na aanbrenging van de hydrogel, terwijl ventriculaire en extracardiale amiodaron weefselconcentraties één tot twee ordes van grootte lager bleven. Desondanks waren epicardiale amiodaronconcentraties in het linker atrium en de ventrikels hoger dan endocardiale concentraties in het rechter atrium, waarschijnlijk ten gevolge van snellere epicardiale dan transmurale distributie, bespoedigd door gelijktijdige medicijnopname en -afgifte via de pericardvloeistof.

Hoofdstuk 5 richt zich op de vraag of aanbrenging van twee-lagige patches (met een op polyethyleenglycol-gebaseerde medicijnbevattende matrix en een op polylactide-co-caprolacton-gebaseerde afdeklaag) een meer atriumspecifieke toediening van geneesmiddelen kunnen bewerkstelligen. De rationale van deze benadering is dat de afdeklaag medicijnlekkage naar de pericardvloeistof minimaliseert. Twee-lagige patches brachten zeer hoge amiodaronconcentraties teweeg in het rechter atrium, terwijl ventriculaire en extracardiale concentraties 2-4 ordes van grootte lager waren gedurende de follow-up periode.

Zowel de hydrogels als de patches veroorzaakten een toename van rechter epicardiale atriale effectieve periodes en geleidingstijden. Daarnaast was de induceerbaarheid van atriale aritmieën onderdrukt vergeleken met de controlegroepen. Deze effecten hielden tot 3 weken aan in de dieren die behandeld werden met hydrogel, terwijl amiodaron-geladen patches nog steeds significante effecten teweeg brachten op het einde van de vier weken-durende experimentele periode.

Hoofdstuk 6 bestudeert epicardiale toediening van amiodaron in een geitenmodel van burst-pacing-geinduceerd persistent atriumfibrilleren. Een nieuw instrument werd gebruikt om gelijktijdige epicardiale en endocardiale electrofysiologische metingen te verrichten om op deze manier de effecten te evalueren van transmurale myocardiale amiodarongradiënten op de atriale electrofysiologie. De initiële resultaten suggereren dat lokale amiodarontoediening effectief is. De aanbrenging 
van de hydrogel verstoorde echter metingen van de epicardiale refractaire periodes en verminderde de signaalkwaliteit. Er is aanvullend onderzoek nodig om de elektrofysiologische effecten van epicardiale amiodarontoediening op geremodeleerde atria op te helderen.

Hoofdstuk 7 bespreekt de belangrijkste bevindingen van dit proefschrift, integreert deze in een breder wetenschappelijk kader, en stipt mogelijke implicaties voor toekomstig onderzoek en klinisch beleid aan.

\section{Conclusies}

Dit proefschrift toont aan dat lokale cardiale farmacotherapie door middel van intrapericardiale en epicardiale toediening van amiodaron de effectiviteit van dit geneesmiddel kan verbeteren en de systemische bijwerkingen kan verminderen. Epicardiale aanbrenging van synthetische, biodegradable polymeren kan langdurige, atrium-specifieke amiodaronafgifte bewerkstelligen. Deze geneesmiddelen toedieningssystemen zouden in de klinische setting toegepast kunnen worden ter preventie van postoperatief atriumfibrilleren. Verdere verbetering van toegangsmethoden en geneesmiddelen toedieningssystemen zou het mogelijk moeten maken om chronische hartziekten te behandelen door middel van epicardiale farmacotherapie. 
DANKWOORD 
Licht aan het eind van de tunnel; het schrijven van dit laatste deel van mijn proefschrift luidt tevens het einde in van een periode waarin veel moest wijken voor de totstandkoming ervan. Daarom wil ik niet alleen diegenen bedanken die aan dit proefschrift bijgedragen hebben, maar ook iedereen die begrip toonde voor mijn tekortkomingen in aandacht en tijd. Een aantal personen zou ik graag in het bijzonder willen noemen en roemen.

In de eerste plaats bedank ik mijn promotor prof. dr. J.G. Maessen. Beste Jos, al vanaf het begin bood jij me de kans om zelf experimenten op te zetten en nieuwe ideeën te ontwikkelen. Ik heb die grote mate van vrijheid en vertrouwen ontzettend weten te waarderen. En wanneer ik af en toe toch tegen een probleem aanliep, kon ik steeds bij jou terecht, ondanks de drukte van je functie als afdelingshoofd. Je brede visie en vermogen om multidisciplinair onderzoek te bevorderen, zijn goed van pas gekomen.

Een tweede onmisbare factor voor de totstandkoming van dit proefschrift is mijn copromotor dr. J.J.R. Hermans. Beste Rob, jij leerde mij de farmacologische principes van intrapericardiale toediening van geneesmiddelen, jij leerde mij het ontwikkelen van een methode om amiodaronconcentraties te bepalen, en jij leerde mij het schrijven van een wetenschappelijk manuscript. Ik heb groot respect voor je kennis, je rationeel denkvermogen en je toegankelijkheid. Maar bovenal heb ik genoten van onze prettige samenwerking.

Veel dank ben ik ook verschuldigd aan dr. F.H. van der Veen. Beste Erik, al die jaren was jij als constante factor betrokken bij elke fase van mijn onderzoek. Jij smeedde mij en mijn collega-promovendi samen tot een hecht team en bracht ons daarmee tot een hoger niveau. Daarnaast liet jij ons inzien dat promoveren meer inhoudt dan alleen maar met onderzoek bezig zijn, namelijk dat het ook een les in levenswijsheid betekent.

Een belangrijke bijdrage aan dit proefschrift is geleverd door prof. dr. U. Schotten. Beste Uli, dankzij jou leerde ik kritisch te zijn met betrekking tot de interpretatie van onderzoeksresultaten. Jij stroomlijnde mijn ideeën over atriale transmurale electrofysiologische heterogeniteit. Bovendien stelde je mij in de gelegenheid om experimenten uit te voeren binnen het kader van je onderzoeksgroep. Ik heb veel waardering voor je professionaliteit en je kwaliteiten als onderzoeker. 
Aan de leden van de beoordelingscommissie, prof. dr. F.W. Prinzen, prof. dr. H.J.G.M. Crijns, prof. dr. J.G.R. de Mey en prof. dr. A. zur Hausen, wil ik mijn dank betuigen voor het beoordelen van dit proefschrift. Ook bedank ik de overige leden van de promotiecommissie voor hun bereidheid mij te opponeren.

Nard, we zijn tegelijkertijd aan het promotie-avontuur begonnen en merkten al meteen dat we op dezelfde golflengte zaten. Met niemand kon ik beter over de uitdagingen (en soms ook frustraties) rondom onderzoek praten, dan met jou. Bedankt voor de mooie momenten; ik denk vooral met veel plezier terug aan onze gezamenlijke reizen, waarbij we altijd een extra dimensie aan het begrip "congresbezoek" toevoegden. En het feit dat jij ook in opleiding bent tot cardioloog, belooft wat voor de toekomst! Antoine, kamergenoot van jou zijn, is een unieke ervaring die ik niet had willen missen. Als er één iemand is die altijd correct en behulpzaam naar zijn medemens is, dan ben jij het wel. En nog bedankt dat jij me telkens (al dan niet bewust) uitkoos als voorproever van je zelfgemaakte baksels; ik beschouwde het altijd als een groot voor(ge)recht! Kris, ik kan het me nog als de dag van gisteren herinneren dat we als jonge promovendi begonnen aan het samenwerkingsproject met DSM en daarbij veel steun aan elkaar hadden. Ik vind het fantastisch dat je ervoor gekozen hebt om je loopbaan voort te zetten als medicus en ik kijk ernaar uit om binnenkort weer collega's te zijn! Loes, ik kan niet zeggen dat mijn (werk)relatie met jou zo goed was als die tussen jou en Nard, maar het kwam toch zeker in de buurt! Bedankt voor de leuke sfeer op de werkvloer en daarbuiten.

Nog iemand aan wie ik veel dank verschuldigd ben, is Monique; met veel enthousiasme en geduld heb jij me geholpen bij mijn onderzoek. En dat geldt ook voor Theo; ik ben blij dat ik nog veel van je heb kunnen leren voordat je met pensioen ging. Daarnaast heb ik tijdens mijn beginperiode als promovendus hulp gekregen van meer ervaren promovendi; Thomas, Koen en Ryan bedankt!

Beste Pol, Tim, Raoel, Stefan, Joyce, Anique, Guido en Marlous, tijdens jullie stage hebben jullie meegeholpen bij de experimenten en laboratoriumanalyses. Daarmee heeft ieder van jullie een steentje bijgedragen aan dit proefschrift en daar ben ik jullie dankbaar voor.

Een woord van dank komt ook toe aan alle leden van de vakgroep Cardiothoracale chirurgie voor de hulp en ondersteuning bij mijn onderzoek. In het bijzonder Lysette: bedankt voor de vlotte en efficiente manier waarop je me secretarieel bijgestaan hebt. 
Dank aan het team van de vakgroep Fysiologie voor de behulpzaamheid in de afgelopen jaren. Sander, Jens, Bart, Dominik, Maura, Sunniva, Arne, Marion en Anniek, bij jullie gaan onderzoek en gastvrijheid hand in hand.

Als wetenschappelijke vertegenwoordigers van DSM waren dr. A.A. Dias, dr. M.J. Boerakker en dr. P. Bruin nauw betrokken bij mijn onderzoek. Beste Aylvin, Mark en Peter, bedankt voor de prettige samenwerking en het beschikbaar stellen van materiaal voor de experimenten.

Nog een persoon die ik graag zou willen bedanken, is prof. dr. L.H. Koole. Als projectleider Bioterials vanuit UM/AZM speelde $u$ een belangrijke rol in het samenwerkingsproject met DSM waarvan mijn onderzoek deel uitmaakte.

Kudos to my Israeli colleagues dr. G. Bolotin and Ziv Beckerman for carrying on the research on epicardial amiodarone delivery.

Tevens bedank ik dr. W. Hinrichs van de Rijksuniversiteit Groningen, afdeling Farmacie, en ook Elise, Maurits en Tonnis Jan voor hun bijdrage aan dit onderzoek.

Veel dank aan de vakgroep Farmacologie voor het beschikbaar stellen van laboratoria, apparatuur en de nuttige adviezen. In het bijzonder Ilona: wat heb jij me goed bijgestaan in het lab!

Ook wil ik de afdeling CPV bedanken voor de goede zorgen en de oprechte betrokkenheid op zowel wetenschappelijk als humaan vlak.

Op de afdeling Pathologie heb ik heel wat uren gespendeerd bij het vriesmicrotoom; dr.ir. J.P.M. Cleutjens en Anique Janssen bedank ik voor de ondersteuning.

Ruimhartig was ook de ontvangst op de afdeling Cardiologie, waar ik met de opleiding startte na het afronden van mijn onderzoek; ik wil al mijn nieuwe collega's bedanken voor de prettige samenwerking tot nu toe.

Alle internisten en assistenten Interne dank ik van harte voor de leerzame en fijne tijd in Venlo. Sander en Loes, bedankt voor het aan huis afzetten, zodat ik net wat extra tijd won om dit proefschrift af te ronden. 
Leuven zal ik voor altijd blijven associeren met de degelijke geneeskunde opleiding, maar ook met het bourgondische leven, mede dankzij mijn studievrienden Camiel, Mari, Geoffrey, Hester, Mark, Linda, Wessel, Kasper en Lieven. Een aantal van jullie is al (bijna) gepromoveerd, wat mij extra motiveerde om dit proefschrift af te ronden; bedankt voor het goede voorbeeld!

Langdurige oprechte vriendschap is iets wat je moet koesteren; Peter, bedankt voor alle leuke momenten en goede gesprekken (op hoger en lager niveau...).

Een proefschrift vormt de apotheose van jarenlang onderzoek en van een noglanger-durende opleiding. Maar de kiem wordt al van jongs af aan gelegd; pap en mam, bedankt hiervoor en ook voor jullie grenzeloze liefde en steun. Leonieke en Wouter, zo verschillend als onze beroepen zijn, zo gelijkgestemd zijn wij; dank voor jullie interesse.

Soms is het nodig om een speciaal iemand op een bijzondere manier te bedanken. De eerste letters van elke alinea in dit dankwoord vormen daarom samen een boodschap voor die persoon. 



\section{ABOUT THE AUTHOR}

Robert Willem Bolderman was born on the 31 $1^{\text {st }}$ of August 1980 in Hoogeveen (The Netherlands). In 1998 he graduated from secondary school (Gymnasium) on Menso Alting College. He studied medicine at the Catholic University of Leuven (Belgium), where he obtained his M.D. degree in 2005 "with high distinction". From 2005 to 2009 he worked as a Ph.D student at the Department of Cardiothoracic Surgery of Maastricht University under supervision of prof. dr. J.G. Maessen. In 2010 he started his clinical cardiology training at the Maastricht University Medical Center under supervision of prof. dr. H.J.G.M. Crijns. Currently, he holds a position as resident in Internal Medicine in VieCuri Medical Center (Venlo, The Netherlands). 


\section{PUBLICATIONS}

\section{Papers}

Bolderman RW, Oyen R, Verrijcken A, Knockaert D, Vanderschueren S. Idiopathic renal infarction. Am J Med. 2006 Apr;119(4):356.e9-12.

Rademakers LM, Gründeman PF, Bolderman RW, van der Veen FH, Maessen JG. Stability of an autologous platelet clot in the pericardial sac: An experimental and clinical study. J Thorac Cardiovasc Surg. 2009;137(5):1190-4.

Bolderman RW, Hermans JJR, Maessen JG. Determination of the class III antiarrhythmic drugs dronedarone and amiodarone, and their principal metabolites in plasma and myocardium by high-performance liquid chromatography and UVdetection. J Chromatogr B Analyt Technol Biomed Life Sci. 2009;877(18-19):1727-31.

Bolderman RW, Hermans JJR, Rademakers LM, Jansen TS, Verheule S, van der Veen FH, Maessen JG. Intrapericardial delivery of amiodarone and sotalol: atrial transmural drug distribution and electrophysiological effects. J Cardiovasc Pharmacol. 2009;54(4):355-63.

Bolderman RW, Bruin P, Hermans JJR, Boerakker MJ, Dias AA, van der Veen FH, Maessen JG. Atrium-targeted drug delivery through an amiodarone-eluting bilayered patch. J Thorac Cardiovasc Surg. 2010;140(4):904-10.

Bolderman RW, Hermans JJR, Rademakers LM, de Jong MM, Bruin P, Dias AA, van der Veen FH, Maessen JG. Epicardial application of an amiodarone-releasing hydrogel to suppress atrial tachyarrhythmias. Int J Cardiol. 2011;149(3):341-6.

\section{Presentations}

Bolderman RW, Hermans JJR, Rademakers LMF, De Jong MMJ, Van der Nagel T, Van der Veen FH, Maessen JG. Is intrapericardial infusion of anti-arrhythmic drugs more effective than intravenous infusion? Nederlandse Vereniging voor Thoraxchirurgie, Amsterdam, 2007.

Bolderman RW, Hermans JJR, Rademakers LMF, Jansen TS, Van der Veen FH, Maessen JG. Amiodarone, in contrast to sotalol, equally decreases epicardial and endocardial atrial fibrillation inducibility, despite a steep transmural concentration gradient. American Heart Association Scientific Sessions, Orlando, Florida, USA, 2007. 
Bolderman RW, Hermans JJR, Rademakers LMF, Jansen TS, De Jong MMJ, Bruin P, Dias AA, Van der Veen FH, Maessen JG. Prophylactic epicardial delivery of amiodarone attenuates atrial fibrillation vulnerability. Nederlandse Vereniging voor Cardiologie, Amsterdam, 2008.

Bolderman RW, Hermans JJR, Rademakers LMF, Jansen TS, De Jong MMJ, Bruin P, Dias AA, Van der Veen FH, Maessen JG. Epicardial amiodarone-releasing hydrogel reduces inducibility of atrial fibrillation in goats. American Heart Association Scientific Sessions, New Orleans, Louisiana, USA, 2008.

Bolderman RW, Bruin P, Hermans JJR, De Jong MMJ, Boerakker MJ, Dias AA, Van der Veen FH, Maessen JG. Amiodarone-eluting patch attenuates atrial fibrillation inducibility in goats. Heart Rhythm Society Scientific Sessions, Boston, Massachusetts, USA, 2009. 